Bull. Soc. math. France

130 (2), 2002, p. 253-307

\title{
PROOF OF NADEL'S CONJECTURE AND DIRECT IMAGE FOR RELATIVE $K$-THEORY
}

\author{
By Alain Berthomieu
}

\begin{abstract}
A "relative" $K$-theory group for holomorphic or algebraic vector bundles on a compact or quasiprojective complex manifold is constructed, and Chern-Simons type characteristic classes are defined on this group in the spirit of Nadel. In the projective case, their coincidence with the Abel-Jacobi image of the Chern classes of the bundles is proved. Some applications to families of holomorphic bundles are given and two Riemann-Roch type theorems are proved for these classes.

RÉSUMÉ (Démonstration d'une conjecture de Nadel et image directe pour la K-théorie relative)

On construit un groupe de $K$-théorie relative pour les fibrés holomorphes ou algébriques sur une variété complexe compacte ou quasiprojective, et des classes caractéristiques de type de Chern-Simons sont définies sur ce groupe dans l'esprit de Nadel. Dans le cas projectif, on démontre la coïncidence de ces classes avec l'image par l'application d'Abel-Jacobi des classes de Chern des fibrés. On donne quelques applications aux familles de fibrés holomorphes et on démontre deux théorèmes de type RiemannRoch pour ces classes.
\end{abstract}

Texte reçu le 12 mars 2001, révisé le 10 décembre 2001

Alain Berthomieu, Laboratoire Émile Picard, UMR CNRS no 5580, UFR MIG, Bat. 1R2, Université Paul Sabatier, 118 route de Narbonne, 31062 Toulouse Cedex 4 (France) E-mail : berthomieu@picard.ups-tlse.fr, berthomieu@ups-albi.fr

2000 Mathematics Subject Classification. — 19E20, 14C17, 19L10, 14C40, 14C30, 32J25, $14 \mathrm{D} 20$.

Key words and phrases. - Relative $K$-theory, holomorphic bundles, characteristic classes, Hodge-Deligne cohomology, Chern-Simons forms, Riemann-Roch theorem. 


\section{Introduction}

On a compact complex manifold $X$, Nadel defined in [35] characteristic classes à la Chern-Weil with values in the odd Dolbeault cohomology of $X$ for triples $(E, F, f)$ where $E$ and $F$ are holomorphic vector bundles and $f: E \stackrel{\sim}{\rightarrow} F$ is a $\mathcal{C}^{\infty}$ isomorphism. He then conjectured that his classes should coïncide with some part of the Abel-Jacobi image of the cycle valued Chern character (without denominators) of the difference $[F]-[E] \in K_{0}(X)$ (here $K_{0}(X)$ is the Grothendieck group of algebraic vector bundles modulo exact sequences).

In this paper, Nadel's theory is widely generalised. Firstly, some relative $K$ theory group $K_{0}^{\mathrm{rel}}(X)$ is constructed (in Section 2) whose elements are equivalence classes of objects $(E, F, f)$ as above; the construction is also valid for couples of $\mathcal{C}^{\infty}$ isomorphic algebraic vector bundles on quasiprojective manifolds. Let $K_{1}(X)$ be Quillen's algebraic $K$-theory group of the category of holomorphic (algebraic) vector bundles on $X, K_{0}^{\mathrm{rel}}(X)$ is then shown to fit in the exact sequence:

$$
K_{1}(X) \stackrel{\mathcal{F}_{*}}{\longrightarrow} K_{1}^{\mathrm{top}}(X) \stackrel{\varphi}{\longrightarrow} K_{0}^{\mathrm{rel}}(X) \stackrel{\partial}{\longrightarrow} K_{0}(X) \stackrel{\mathcal{F}_{*}}{\longrightarrow} K_{0}^{\mathrm{top}}(X) .
$$

Secondly, let $F_{r(L)}($ for $1 \leq r \leq \operatorname{dim} X)$ be the subspaces of the space $\wedge_{(L)}(X)$ of (logarithmic) $\mathcal{C}^{\infty}$ differential forms on $X$ defining the Hodge filtration of the (logarithmic) de Rham complex of $X$ (see Subsection 3.2, logarithmic forms correspond to the quasiprojective case). Chern-Simons transgression between two compatible connexions on $E$ and $F$ is shown (in Section 3 ) to provide characteristic class morphisms:

$$
\begin{gathered}
\mathcal{N}_{P}: K_{0}^{\mathrm{rel}}(X) \longrightarrow H^{2 \bullet-1}\left(\wedge_{(L)}(X) / F_{\bullet}(L), d\right), \\
\mathcal{M}_{P}: K_{0}^{\mathrm{rel}}(X) \longrightarrow H^{2 \bullet-1}\left(\wedge_{(L)}(X) / F_{\bullet}(L), d\right),
\end{gathered}
$$

for an additive or multiplicative invariant polynomial $P$ respectively (in the second line, the group structure of $H^{2 \bullet-1}\left(\wedge(X) / F_{\bullet}, d\right)$ need not be the usual addition if $X$ is a non Kähler or non compact complex analytic manifold). In particular for the total Chern class $P=c_{\text {tot }}$

$$
\mathcal{M}_{c_{\text {tot }}}(E, F, f)=\overline{c_{\text {tot }}}\left(\nabla_{E}, f^{*} \nabla_{F}\right) \wedge c_{\text {tot }}^{-1}\left(\nabla_{F}^{2}\right)
$$

where $\nabla_{E}$ and $\nabla_{F}$ are connexions on $E$ and $F$ compatible with their holomorphic (algebraic) structure, $c_{\text {tot }}^{-1}\left(\nabla_{F}^{2}\right)$ is the Chern-Weil form calculated from the curvature of $\nabla_{F}$ and $\overline{c_{\text {tot }}}\left(\nabla_{E}, f^{*} \nabla_{F}\right)$ is the Chern-Simons transgression form on $X$ such that

$$
d \overline{c_{\mathrm{tot}}}\left(\nabla_{E}, f^{*} \nabla_{F}\right)=c_{\mathrm{tot}}\left(\left(f^{*} \nabla_{F}\right)^{2}\right)-c_{\mathrm{tot}}\left(\nabla_{E}^{2}\right)
$$

TOME $130-2002-\mathrm{N}^{\mathrm{O}} 2$ 
Let $H_{\mathcal{D}}^{2 \bullet}(X, \mathbb{Z}(\bullet))$ be Hodge-Deligne(-Beilinson) cohomology groups (see Subsection 3.2), the class $\mathcal{M}_{c_{\text {tot }}}$ is proved to fit in the commutative diagram:

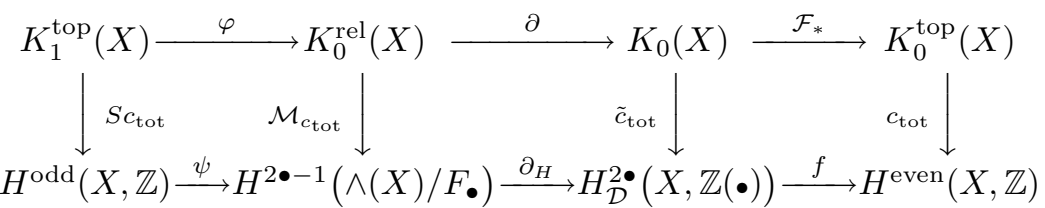

(the second line is a classical exact sequence associated to Hodge-Deligne cohomology [17], Cor. 2.10 b).

An analogue construction is performed by Karoubi in [28], $\S 6$, for flat $A$ bundles, where $A$ is a Banach algebra; this could be related to the theory developped here since the category of vector bundles on $X$ has same algebraic $K$-theory groups as the ring of algebraic functions on some affine variety $\widetilde{X}$ related to $X[26]$ (see [30], §4.1) but the constructions here are much nearer to Nadel's ideas and more adapted to study examples and direct images. However, Karoubi's multiplicative $K$-theory as treated in [30] fits nicely to the theory developped here as adding an intermediary line in the diagram above (see (29) below). The reason why higher $K$-theory is not studied here is that higher algebraic $K$-theory groups are difficult to describe explicitly (see [30], §4).

Nadel's results on his characteristic classes now generalise as follows:

The integrality property (see [35], $\S 6,11$ and 12) is recovered by the commutativity of the "left" square of the above diagram.

Let $K_{0}^{\text {def }}$ be the subgroup of $K_{0}^{\text {rel }}$ generated by deformations of holomorphic vector bundles, (see definition 5.1) and $F_{r-1} H^{2 r-1}\left(\wedge(X) / F_{r}\right)$ the subgroup of classes in $H^{2 r-1}\left(\wedge(X) / F_{r}\right)$ represented by differential forms lying in $F_{r-1}$, the rigidity result of Nadel (see [35], $\S 5,10$ and 12) reads now as follows: the image of $K_{0}^{\text {def }}(X)$ under $\mathcal{N}_{P}$ or $\mathcal{M}_{P}$ (for any $P$ ) is included in $F_{\bullet-1} H^{2 \bullet-1}\left(\wedge(X) / F_{\bullet}\right)$ (this equals $\bigoplus_{p} H^{p, p+1}(X)$ if $X$ is compact Kähler), this result allows to precise Nadel's "topological monodromy restrictions"; these restrictions are generalised to higher order topological $K$-theory groups of $X$ in Remark 5.7 below, and the rigidity result is generalised in Proposition 5.6 to reprove a statement by Esnault and Srinivas [16], Prop. 1, about vector bundles with holomorphic connexions.

For $1 \leq r \leq \operatorname{dim} X$, let $J_{r}(X)$ denote Griffiths' $r$-th intermediate jacobian

$$
J_{r}(X):=H^{2 r-1}\left(\wedge(X) / F_{r}, d\right) / \psi\left(H^{2 r-1}(X, \mathbb{Z})\right)
$$

define

$$
K_{0}^{\mathrm{hom}}=\operatorname{Ker}\left(K_{0} \stackrel{\mathcal{F}_{*}}{\longrightarrow} K_{0}^{\mathrm{top}}\right) \cong K_{0}^{\mathrm{rel}} / \varphi\left(K_{1}^{\mathrm{top}}\right)
$$

BULletin DE LA SOCIÉtÉ MATHÉMATIQUE DE FRANCE 
and call $K_{0}^{\text {cont }}$ the trace of $K_{0}^{\text {def }}$ in $K_{0}^{\text {hom }}$. Then any holomorphic bundle on $\mathcal{M} \times X$ where $\mathcal{M}$ is any pointed complex manifold gives rise to a map

$$
\mathcal{M} \longrightarrow K_{0}^{\text {cont }} \stackrel{\mathcal{M}_{c_{\text {tot }}}}{\longrightarrow} J_{r}(X)
$$

this map is proved to be holomorphic in $\S 5.3$ below, and its degree one part is (as Nadel proves it in [35], §9) the classical map $\mathcal{M} \rightarrow J_{1}(X)$ associated to the determinant line bundle. This leads to the question of the comparison of this map with the Albanese map of moduli spaces of vector bundles on $X$. The examples of abelian surfaces and of the cubic threefold are studied in $\S \S 6.5$ and 5.3.

For $X$ projective, let $\mathrm{CH}(X)$ denote the Chow ring of algebraic cycles in $X$ modulo rational equivalence, and $\mathrm{CH}_{\text {hom }}(X)$ its subgroup consisting of cycles homologically equivalent to zero, i.e. $\mathrm{CH}_{\text {hom }}(X)$ is the kernel of the cycle map $\mathrm{CH}(X) \rightarrow H^{\text {even }}(X, \mathbb{Z})$. The Chern isomorphism

$$
K_{0}(X) \otimes \mathbb{Q} \cong \mathrm{CH}(X) \otimes \mathbb{Q}
$$

(induced by the cycle valued Chern character) makes $K_{0}^{\text {hom }}(X) \otimes \mathbb{Q}$ correspond to $C H_{\text {hom }}(X) \otimes \mathbb{Q}$. Let

$$
A J: C H_{\mathrm{hom}}(X) \longrightarrow \bigoplus_{r} J_{r}(X)
$$

denote the Abel-Jacobi map (see for example [31], Lecture 12). The conjecture of Nadel [35], $\S 13$, is then a consequence of the following

Theorem 1.1. - If $X$ is projective, the following diagram commutes

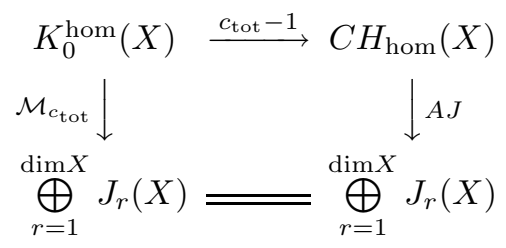

where 1 denotes the unit (of intersection) in the Chow ring $\mathrm{CH}(X)$ and $c_{\mathrm{tot}}$ is the cycle valued total Chern class [23].

This result means that $\mathcal{M}_{c_{\text {tot }}}$ provides an analogue of the Abel-Jacobi map on $K_{0}^{\text {hom }}$ (which can be defined on nonprojective manifolds). In particular the above question about the Albanese map of moduli spaces is closely related to the conjectural universality of the Abel-Jacobi map as a map from $\mathrm{CH}_{\text {hom }}$ to Abelian varieties.

This theorem is proved in Section 4: the compatibility of Chern classes with the cycle class in Deligne-Beilinson cohomology (see [17], §8), and between the latter cycle class with the Abel-Jacobi map (see [19], [15], [17], §7.12, [25], Lemma 1.22 and [21], Thm. 3.5) being granted, this theorem becomes a consequence of the commutativity of the "central" square of diagram (2) above; in fact

TOME $130-2002-\mathrm{N}^{\mathrm{O}} 2$ 
once Nadel's classes have been recognized to consist of Chern-Simons transgression forms, the compatibility between Chern classes in Cheeger-Simons characters and in Hodge-Deligne-Beilinson cohomology as studied by Brylinski [11], Zucker [39], Karoubi [30] and Gillet-Soulé [21] gives the answer.

Finally in Section 6, direct image questions are considered for relative $K$ theory: firstly, a morphism $K_{0}^{\mathrm{rel}}(X) \rightarrow K_{0}^{\mathrm{rel}}(Y)$ is constructed for any proper submersion $\pi: X \rightarrow Y$ of quasiprojective varieties. This needs resolutions to the right in $K_{0}^{\mathrm{rel}}(X)$ by triples $(A, B, g)$ where $A$ and $B$ are $\pi$-acyclic in the sense that $R^{j} \pi_{*} A$ and $R^{j} \pi_{*} B$ vanish for all $j \geq 1$. A trick from [2] (Prop. 2.2, see also [3], §9) is then needed to define the direct image of such a $(A, B, g)$ because the $\bar{\partial}+\bar{\partial}^{*}$ operators associated to connexions on $A$ realising some homotopy between two compatible connexions on $A$ and $B$ respectively need not have kernels of constant dimension. A Riemann-Roch type theorem for $\mathcal{N}_{\mathrm{ch}}$ is then proved in the projective case: it is obtained by "integrating along $[0,1]$ " the refinement (at the level of differential forms) of the families index theorem on $X \times[0,1]$ taken for the $\bar{\partial}+\bar{\partial}^{*}$ operator on vector bundles which may be nonholomorphic. On $X \times\{0\}$ and $X \times\{1\}$ however, the vector bundles are holomorphic and the double transgression of the families index theorem of Bismut and Köhler [9] plays a crucial role. This Riemann-Roch theorem is then applied to recognize the map $\mathcal{M}_{c_{\text {tot }}}$ in Yoshioka's construction of the Albanese map for moduli spaces of vector bundles on Abelian surfaces [38], [37].

Secondly for a closed immersion of projective varieties $\iota: Y \rightarrow X$, the direct image morphism is made available only from $K_{0}^{\operatorname{def}}(Y)$ to $K_{0}^{\operatorname{def}}(X)$; this is because $\mathcal{C}^{\infty}$ isomorphisms do not fit nicely with with resolutions of vector bundles on $Y$ by vector bundles on $X$. The Riemann-Roch statement is obtained from Bismut Gillet and Soulé's double transgression for immersions [8]. Note that these Riemann-Roch results are not consequences of the "usual" Riemann-Roch theorem on Deligne-Beilinson cohomology proved by Gillet [18] because Gillet's result cannot take more in consideration than $K_{0}^{\text {hom }}(X)$ in the case of closed immersions and $K_{0}^{\text {hom }}(X) \otimes \mathbb{Q}$ in the general case.

ACKnowledgements. - I am much indebted to D. Delabre for indicating the references [30], [28] and [29], and to Ph. Eyssidieux, J. Tapia, and again D. Delabre for helpful discussions and comments.

\section{Relative $K$-theory}

Two cases are considered here: $X$ is either a smooth quasiprojective complex manifold or a complex analytic manifold. The results will be stated in the first case (the second case is deduced by replacing everywhere "algebraic" by "holomorphic"). In 2.1 , the "relative" $K$-theory group $K_{0}^{\text {rel }}(X)$ is defined, it 
is the relevant source for Nadel's classes. In 2.2, this group is shown to fit in the exact sequence (1).

2.1. Relative $\boldsymbol{K}$-theory. - Let $\mathcal{E}^{\text {rel }}(X)$ the free abelian group generated by triples $(E, F, f)$ where $E$ and $F$ are algebraic vector bundles on $X$ and $f: E \rightarrow F$ is a $\mathcal{C}^{\infty}$ isomorphism.

Definition. - $K_{0}^{\mathrm{rel}}(X)$ is the quotient of $\mathcal{E}^{\mathrm{rel}}(X)$ by the following relations:

(i) $(E, F, f)=0$ if $f$ is an algebraic isomorphism;

(ii) $(E, E, f)=0$ if $f$ is $\mathcal{C}^{\infty}$-isotopic to the identity of $E$ (isotopic means hereafter homotopic throw isomorphisms);

(iii) $(E, F, f)+(G, H, h)=(E \oplus G, F \oplus H, f \oplus h)$;

(iv) $\left(E, E^{\prime} \oplus E^{\prime \prime}, s \oplus p\right)=0$ for any short (algebraic) exact sequence:

$$
0 \rightarrow E^{\prime} \stackrel{i}{\longrightarrow} E \stackrel{p}{\longrightarrow} E^{\prime \prime} \rightarrow 0
$$

of vector bundles on $X$ and any $\mathcal{C}^{\infty}$ morphism $s: E \rightarrow E^{\prime}$ such that $s \circ i$ is the identity of $E^{\prime}$ (such a morphism will be called hereafter a " $\mathcal{C}^{\infty}$-left inverse" of $i$ ).

Proposition 2.1. - The following relations hold in $K_{0}^{\mathrm{rel}}(X)$ :

$(\mathrm{v}):(E, F, f)=(E, F, g)$ if $f$ and $g$ are $\mathcal{C}^{\infty}$-isotopic;

(vi) : $(E, F, f)+(F, G, g)=(E, G, g \circ f)\left(\right.$ one has $(E, F, f)=-\left(F, E, f^{-1}\right)$ in particular);

(vii) : $(E, E, f)=\left(E \oplus G, E \oplus G, f \oplus \operatorname{Id}_{G}\right) \quad$ for any $\mathcal{C}^{\infty}$ vector bundle $G$ on $X$ such that $E \oplus G$ admits some algebraic structure (in particular the element $(E, E, f)$ of $K_{0}^{\mathrm{rel}}(X)$ does not depend on the algebraic structure of $E)$;

(viii) : for a commutative diagram with (algebraic) exact lines as

(3)

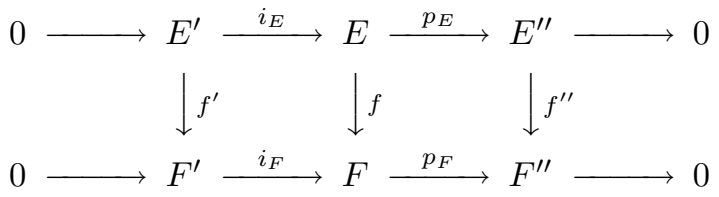

and whose "vertical" maps are $\mathcal{C}^{\infty}$ isomorphisms, then

$$
(E, F, f)=\left(E^{\prime}, F^{\prime}, f^{\prime}\right)+\left(E^{\prime \prime}, F^{\prime \prime}, f^{\prime \prime}\right)
$$

Proof. - It is a standard fact that $E \oplus F \stackrel{f \oplus f^{-1}}{\longrightarrow} F \oplus E$ and $E \oplus F \stackrel{\text { Id }}{\longrightarrow} E \oplus F$ are $\mathcal{C}^{\infty}$-isotopic (of course modulo the canonical identification $E \oplus F \stackrel{\sim}{\rightarrow} F \oplus E$ ). This with (ii) and (iii) proves the special case of (vi). $E \oplus F \stackrel{f \oplus g^{-1}}{\longrightarrow} F \oplus E$ and $E \oplus F \stackrel{g^{-1} \circ f \oplus \operatorname{Id}_{F}}{\longrightarrow} E \oplus F$ are also $\mathcal{C}^{\infty}$-isotopic, this with (ii), (iii) and the 
special case of (vi) proves (v). $E \oplus F \stackrel{f \oplus g}{\longrightarrow} F \oplus G$ and $E \oplus F \stackrel{g \circ f \oplus \operatorname{Id}_{F}}{\longrightarrow} G \oplus F$ are $\mathcal{C}^{\infty}$-isotopic, so that (v) and (iii) imply (vi) in full generality.

Furthermore $E \oplus(E \oplus G) \stackrel{f^{-1} \oplus\left(f \oplus \operatorname{Id}_{G}\right)}{\longrightarrow} E \oplus(E \oplus G)$ is isotopic to the identity of $E \oplus(E \oplus G)$. This with (iii) and (v) proves (vii). Finally if $s_{E}$ and $s_{F}$ are $\mathcal{C}^{\infty}$ left inverses of $i_{E}$ and $i_{F}$ of diagram (3), one has from (vi), (iii) and (iv):

$$
\begin{aligned}
(E, F, f) & =\left(E, E^{\prime} \oplus E^{\prime \prime}, s_{E} \oplus p_{E}\right)+\left(E^{\prime} \oplus E^{\prime \prime}, F^{\prime} \oplus F^{\prime \prime}, f^{\prime} \oplus f^{\prime \prime}\right)+ \\
& +\left(F^{\prime} \oplus F^{\prime \prime}, F,\left(s_{F} \oplus p_{F}\right)^{-1}\right) \\
= & \left(E^{\prime}, F^{\prime}, f^{\prime}\right)+\left(E^{\prime \prime}, F^{\prime \prime}, f^{\prime \prime}\right)
\end{aligned}
$$

Of course this generalises to longer $\mathcal{C}^{\infty}$ isomorphic exact sequences.

REMARK. - $K_{0}^{\mathrm{rel}}(X)$ can be defined using only relations (i), (ii) and (viii) which together are equivalent to (i), (ii), (iii) and (iv).

2.2. The exact sequence. - Remember that the topological $K$-theory group $K_{1}^{\text {top }}(X)$ is generated by $\mathcal{C}^{\infty}$ automorphisms $\alpha: T_{N} \rightarrow T_{N}$ of trivial bundles $T_{N}$ (of any rank $N$ ) modulo the following relations (see [27], $\S \S 3.2$ to 3.8 of Chapter II):

(1) $\left(T_{N}, \alpha\right)+\left(T_{M}, \beta\right)=\left(T_{N} \oplus T_{M}, \alpha \oplus \beta\right)$;

(2) $\left(T_{N}, \alpha\right)+\left(T_{N}, \beta\right)=\left(T_{N}, \beta \circ \alpha\right)$ (in particular $\left.\left(T_{N}, \operatorname{Id}\right)=0\right)$;

(3) $\left(T_{N}, \alpha\right)=\left(T_{N}, \beta\right) \quad$ if $\alpha$ and $\beta$ are $\mathcal{C}^{\infty}$-isotopic.

(In fact (2) is a consequence of (1) and (3).)

The following description of Quillen's $K_{1}$-group of the category of algebraic vector bundles is due to Gillet and Grayson [20], §5: elements are couples of algebraic vector bundles $(E, F)$ each of which is endowed with two admissible filtrations:

$$
\begin{aligned}
& 0=E_{0} \subset E_{1} \subset \cdots \subset E_{p}=E \quad \text { and } \quad 0=E_{0}^{\prime} \subset E_{1}^{\prime} \subset \cdots \subset E_{q}^{\prime}=E, \\
& 0=F_{0} \subset F_{1} \subset \cdots \subset F_{p}=F \quad \text { and } \quad 0=F_{0}^{\prime} \subset F_{1}^{\prime} \subset \cdots \subset F_{q}^{\prime}=F,
\end{aligned}
$$

with algebraic isomorphisms for any $1 \leq i \leq p$ and $1 \leq j \leq q$ :

$$
f_{i}: E_{i} / E_{i-1} \stackrel{\sim}{\longrightarrow} F_{i} / F_{i-1} \quad \text { and } f_{j}^{\prime}: E_{j}^{\prime} / E_{j-1}^{\prime} \stackrel{\sim}{\longrightarrow} F_{j}^{\prime} / F_{j-1}^{\prime}
$$

Such an element of $K_{1}(X)$ will be denoted by $(E, F ; \cong)$. The $f_{i}$ (resp. the $f_{j}^{\prime}$ ) yield a isotopy class of $\mathcal{C}^{\infty}$ isomorphism which will be denoted by $E \stackrel{f}{\rightarrow} F$ (resp. $E \stackrel{f^{\prime}}{\rightarrow} F$ ). The natural map $\mathcal{F}_{*}: K_{1}(X) \rightarrow K_{1}^{\text {top }}(X)$ is given by:

$$
(E, F, \cong) \longmapsto\left(E \oplus G, f^{\prime-1} \circ f \oplus \operatorname{Id}_{G}\right)
$$

where $G$ is any $\mathcal{C}^{\infty}$ vector bundle such that $E \oplus G$ is topologically trivial.

BULLETIN DE LA SOCIÉtÉ MATHÉMATIQUE DE FRANCE 
Consider now the following maps:

$$
\begin{array}{ll}
K_{0}^{\mathrm{rel}}(X) \stackrel{\partial}{\longrightarrow} K_{0}(X), & K_{1}^{\mathrm{top}}(X) \stackrel{\varphi}{\longrightarrow} K_{0}^{\mathrm{rel}}(X), \\
(E, F, f) \longmapsto[F]-[E], & \left(T_{N}, \alpha\right) \longmapsto\left(T_{N}, T_{N}, \alpha\right),
\end{array}
$$

where $[E]$ stands for the class of the algebraic vector bundle $E$ in $K_{0}(X)$.

Proposition 2.2. - These maps are well defined and give rise to the exact sequence (1):

$$
K_{1}(X) \stackrel{\mathcal{F}_{*}}{\longrightarrow} K_{1}^{\mathrm{top}}(X) \stackrel{\varphi}{\longrightarrow} K_{0}^{\mathrm{rel}}(X) \stackrel{\partial}{\longrightarrow} K_{0}(X) \stackrel{\mathcal{F}_{*}}{\longrightarrow} K_{0}^{\mathrm{top}}(X)
$$

where the last map is provided by forgetting the algebraic structure.

Proof. - Clearly relations defining $K_{1}^{\text {top }}(X)$ are sent to relations in $K_{0}^{\text {rel }}(X)$ and relations in $K_{0}^{\text {rel }}(X)$ are sent to relations in $K_{0}(X)$. Also it should be clear that this is a complex: $\varphi \circ \mathcal{F}_{*}(E, F, \cong)$ vanishes because in $K_{0}^{\mathrm{rel}}(X)$ :

$$
\left(E \oplus G, E \oplus G, f^{\prime-1} \circ f \oplus \operatorname{Id}_{G}\right)=(E, F, f)-\left(E, F, f^{\prime}\right)
$$

and repeated use of property (iv) (and (i)) above shows that

$$
(E, F, f)=\sum_{1 \leq i \leq p}\left(E_{i} / E_{i-1}, F_{i} / F_{i-1}, f_{i}\right)=0
$$

and similarly for $\left(E, F, f^{\prime}\right)$.

Any element of $K_{0}(X)$ can be represented by some difference $[F]-[E]$. If such an element has vanishing image in $K_{0}^{\text {top }}(X)$, this means that there exists some trivial bundle $T_{N}$ on $X$ such that $E \oplus T_{N}$ and $F \oplus T_{N}$ are isomorphic as $\mathcal{C}^{\infty}$ vector bundles. Let $\alpha$ be such an isomorphism, then clearly

$$
[F]-[E]=\partial\left(E \oplus T_{N}, F \oplus T_{N}, \alpha\right) .
$$

Any element of $K_{0}^{\text {rel }}(X)$ can be represented by some triple $(E, F, f)$. If such a triple has vanishing image in $K_{0}(X)$, then there exist two exact sequences

$$
0 \rightarrow G^{\prime} \stackrel{i_{G}}{\longrightarrow} G \stackrel{p_{G}}{\longrightarrow} G^{\prime \prime} \rightarrow 0 \quad \text { and } \quad 0 \rightarrow H^{\prime} \stackrel{i_{H}}{\longrightarrow} H \stackrel{p_{H}}{\longrightarrow} H^{\prime \prime} \rightarrow 0
$$

of algebraic vector bundles on $X$ such that

$$
E \oplus G \oplus H^{\prime} \oplus H^{\prime \prime} \cong F \oplus G^{\prime} \oplus G^{\prime \prime} \oplus H \quad(=: K)
$$

are isomorphic as algebraic vector bundles. Let $s_{G}: G \rightarrow G^{\prime}$ and $s_{H}: H \rightarrow H^{\prime}$ be $\mathcal{C}^{\infty}$ left inverses of $i_{G}$ and $i_{H}$ respectively, then in $K_{0}^{\mathrm{rel}}(X)$ :

$$
(E, F, f)=\left(K, K, f \oplus\left(s_{G} \oplus p_{G}\right) \oplus\left(s_{H} \oplus p_{H}\right)^{-1}\right) .
$$

Now let $L$ be any $\mathcal{C}^{\infty}$ bundle such that $K \oplus L$ is the trivial bundle $T_{N}$ (for some $N$ ). Using relation (vii) in $K_{0}^{\mathrm{rel}}(X)$ one obtains

$$
(E, F, f)=\varphi\left(K \oplus L, f \oplus\left(s_{G} \oplus p_{G}\right) \oplus\left(s_{H} \oplus p_{H}\right)^{-1} \oplus \operatorname{Id}_{L}\right) .
$$

TOME $130-2002-\mathrm{N}^{\mathrm{O}} 2$ 
Finally if $\varphi\left(T_{N}, g\right)$ vanishes in $K_{0}^{\mathrm{rel}}(X)$, then there exists two exact sequences

$$
0 \rightarrow G^{\prime} \stackrel{i_{G}}{\longrightarrow} G \stackrel{p_{G}}{\longrightarrow} G^{\prime \prime} \rightarrow 0 \quad \text { and } \quad 0 \rightarrow H^{\prime} \stackrel{i_{H}}{\longrightarrow} H \stackrel{p_{H}}{\longrightarrow} H^{\prime \prime} \rightarrow 0
$$

two $\mathcal{C}^{\infty}$ left inverses $s_{G}: G \rightarrow G^{\prime}$ and $s_{H}: H \rightarrow H^{\prime}$ of $i_{G}$ and $i_{H}$ and some algebraic vector bundle $K$ for which

$$
G^{\prime} \oplus G^{\prime \prime} \oplus H \cong G \oplus H^{\prime} \oplus H^{\prime \prime} \cong T_{N} \oplus K
$$

as algebraic vector bundles, and $g \oplus \operatorname{Id}_{K}$ and $\left(p_{G} \oplus s_{G}\right)^{-1} \oplus\left(p_{H} \oplus s_{H}\right)$ are $\mathcal{C}^{\infty}$ isotopic. Then using the following filtrations

$$
\begin{aligned}
& 0 \subset T_{N} \oplus K \quad \text { and } \quad 0 \subset G^{\prime} \subset G^{\prime} \oplus G^{\prime \prime} \subset G^{\prime} \oplus G^{\prime \prime} \oplus H^{\prime} \subset G^{\prime} \oplus G^{\prime \prime} \oplus H, \\
& 0 \subset T_{N} \oplus K \quad \text { and } \quad 0 \subset G^{\prime} \subset G \subset G \oplus H^{\prime} \subset G \oplus H^{\prime} \oplus H^{\prime \prime} \text {, }
\end{aligned}
$$

with isomorphisms associated to the exact sequences above, one gets

$$
\left(T_{N}, g\right)=\mathcal{F}_{*}\left(T_{N} \oplus K, T_{N} \oplus K, \cong\right) .
$$

Definition. - Let $K_{0}^{\mathrm{hom}}(X)$ be the following quotient of $K_{0}^{\mathrm{rel}}(X)$ :

$$
K_{0}^{\mathrm{hom}}(X):=K_{0}^{\mathrm{rel}}(X) / \varphi\left(K_{1}^{\mathrm{top}}(X)\right) \cong \operatorname{Im} \partial=\operatorname{Ker} \mathcal{F}_{*} \subset K_{0}(X) .
$$

Let $\mathrm{CH}(X)$ be the full Chow group of cycles in $X$ modulo rational equivalence, and $\gamma$ the cycle map given by integration on the cycle, one has the commutative diagram:

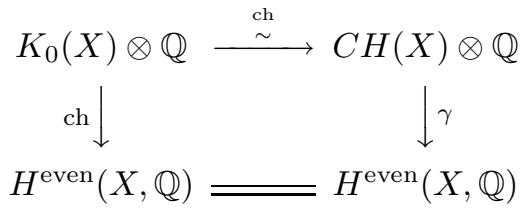

The "top horizontal" Chern character map provides an isomorphism so that it induces an isomorphism between the kernels of the two vertical maps, namely

$$
K_{0}^{\text {hom }}(X) \otimes \mathbb{Q} \cong \mathrm{CH}_{\text {hom }}(X) \otimes \mathbb{Q}
$$

where $\mathrm{CH}_{\text {hom }}(X)$ is the group of cycles homologically equivalent to 0 .

\section{Characteristic classes on relative $K$-theory}

This section is organised as follows: basic facts about Chern-Simons transgression theory are recalled in 3.1 and considerations on cohomology groups of quasiprojective manifolds are made in 3.2 , from which characteristic classes à la Nadel are constructed on $K_{0}^{\text {rel }}(X)$ for additive and multiplicative polynomials and relations between then are established in 3.3 (the link between these classes and previous works by Karoubi [29], [30], Brylinski [11] and Zucker [39] will be made explicit in the next section). 
3.1. Chern-Simons transgressions. - Let $P$ be any invariant polynomial on $\operatorname{GL}(n, \mathbb{C})$. To any connexion $\nabla$ (of curvature $\nabla^{2}$ ) on a rank $n \mathcal{C}^{\infty}$ vector bundle $\xi$ on $X$, Chern-Weil theory associates the differential form $P\left(\nabla^{2}\right)$ on $X$ which is of even degree, closed, and whose de Rham cohomology class coïncides with the one of $\xi$ 's topological characteristic class associated to $P$.

For two connexions $\nabla_{0}$ and $\nabla_{1}$ on the same bundle $\xi$ and any homotopy $\left(\nabla_{t}\right)_{t \in[0,1]}$ linking them, consider the connexion

$$
\widetilde{\nabla}=\mathrm{d} t \wedge \frac{\partial}{\partial t}+\nabla_{t}
$$

on the bundle $p_{2}^{*} \xi$ on $[0,1] \times X$ (where $p_{2}:[0,1] \times X \rightarrow X$ is the projection on the second factor) and the differential form on $[0,1] \times X$

(4) $P\left(\widetilde{\nabla}^{2}\right)=P\left(\nabla_{t}^{2}\right)+\mathrm{d} t \wedge \frac{\partial}{\partial b} P\left(\nabla_{t}^{2}+b \frac{\partial \nabla_{t}}{\partial t}\right)_{b=0}=P\left(\nabla_{t}^{2}\right)+\mathrm{d} t \wedge P^{\prime}\left(\nabla_{t}^{2} ; \frac{\partial \nabla_{t}}{\partial t}\right)$

where $P^{\prime}(A ; B)$ denotes the differential of $P$ at the matrix $A$ evaluated on $B$. This form is closed by Chern-Weil theory so that:

$$
\frac{\partial}{\partial t} P\left(\nabla_{t}^{2}\right)=\mathrm{d} P^{\prime}\left(\nabla_{t}^{2} ; \frac{\partial \nabla_{t}}{\partial t}\right)
$$

where $\mathrm{d}$ in the right hand side stands for the exterior differential along $X$.

Definition. - The Chern-Simons transgression form is defined to be

$$
\bar{P}\left(\nabla_{0}, \nabla_{1}\right)=\int_{[0,1]} P\left(\widetilde{\nabla}^{2}\right)=\int_{[0,1]} \mathrm{d} t \wedge P^{\prime}\left(\nabla_{t}^{2} ; \frac{\partial \nabla_{t}}{\partial t}\right) .
$$

(Note for sign conventions that for any odd degree form $\alpha$ on $X$

$$
\int_{[0,1]} \mathrm{d} t \wedge \alpha=\alpha=-\int_{[0,1]} \alpha \wedge \mathrm{d} t \quad \text { and } \quad \mathrm{d} \int_{[0,1]} \mathrm{d} t \wedge \beta(t)=\int_{[0,1]} \mathrm{d} t \wedge d \beta(t)
$$

for any (possibly $t$ dependent) form $\beta(t)$ on $X$.)

I recall now the standard properties of $\bar{P}\left(\nabla_{0}, \nabla_{1}\right)$.

Let $\wedge(X)$ be the space of $\mathcal{C}^{\infty}$ differential forms (of any degree) on $X$

Proposition 3.1. - $\bar{P}\left(\nabla_{0}, \nabla_{1}\right)$ depends on the chosen homotopy $\left(\nabla_{t}\right)_{t \in[0,1]}$ only throw an exact form, so that it defines a class in $\wedge(X) / \mathrm{d} \wedge(X)$, which is functorial by pull-backs and verifies

$$
\begin{aligned}
\mathrm{d} \bar{P}\left(\nabla_{0}, \nabla_{1}\right) & =P\left(\nabla_{1}^{2}\right)-P\left(\nabla_{0}^{2}\right), \\
\bar{P}\left(\nabla_{0}, \nabla_{1}\right) & =-\bar{P}\left(\nabla_{1}, \nabla_{0}\right) \quad \text { in } \quad \wedge(X) / \mathrm{d} \wedge(X), \\
\bar{P}\left(\nabla_{0}, \nabla_{2}\right) & =\bar{P}\left(\nabla_{0}, \nabla_{1}\right)+\bar{P}\left(\nabla_{1}, \nabla_{2}\right) \quad \text { in } \wedge(X) / \mathrm{d} \wedge(X),
\end{aligned}
$$

TOME $130-2002-\mathrm{N}^{\mathrm{O}} 2$ 
for any connexions $\nabla_{0}, \nabla_{1}$, and $\nabla_{2}$ on the same bundle $\xi$.

$$
\begin{aligned}
\overline{P \wedge Q}\left(\nabla_{0}, \nabla_{1}\right) & =\bar{P}\left(\nabla_{0}, \nabla_{1}\right) \wedge Q\left(\nabla_{1}^{2}\right)+P\left(\nabla_{0}^{2}\right) \wedge \bar{Q}\left(\nabla_{0}, \nabla_{1}\right) \\
& =P\left(\nabla_{1}^{2}\right) \wedge \bar{Q}\left(\nabla_{0}, \nabla_{1}\right)+\bar{P}\left(\nabla_{0}, \nabla_{1}\right) \wedge Q\left(\nabla_{0}^{2}\right) \\
& \text { in } \wedge(X) / \mathrm{d} \wedge(X) .
\end{aligned}
$$

The class of $\bar{P}\left(\nabla_{0}, \nabla_{1}\right)$ is locally gauge invariant (which means that if $f$ is a $\mathcal{C}^{\infty}$ automorphism of the bundle which is isotopic to the identity then one has $\left.\bar{P}\left(\nabla_{0}, \nabla_{1}\right)=\bar{P}\left(\nabla_{0}, f^{*} \nabla_{1}\right)\right)$.

Here $P \wedge Q$ is the polynomial obtained from $P$ and $Q$ throw exterior product of forms (this corresponds to cup product in cohomology).

Proof of the last statement. - If $\left(f_{t}\right)_{t \in[0,1]}$ is a $\mathcal{C}^{\infty}$ homotopy of automorphisms of $E$ and $\nabla$ a connexion on $E$ then

$$
\frac{\partial}{\partial t}\left(f_{t}^{*} \nabla\right)=\frac{\partial}{\partial t}\left(f_{t}^{-1} \circ \nabla \circ f_{t}\right)=f_{t}^{-1} \circ\left[\nabla, \frac{\partial f_{t}}{\partial t} f_{t}^{-1}\right] \circ f_{t} .
$$

Using the $\operatorname{GL}(n, \mathbb{C})$-invariance of $P$, one then obtains

$$
\begin{aligned}
P^{\prime}\left(\left(f_{t}^{*} \nabla\right)^{2} ; \frac{\partial}{\partial t}\left(f_{t}^{*} \nabla\right)\right) & =P^{\prime}\left(\nabla^{2} ;\left[\nabla, \frac{\partial f_{t}}{\partial t} f_{t}^{-1}\right]\right) \\
& =\mathrm{d}\left[P^{\prime}\left(\nabla^{2} ; \frac{\partial f_{t}}{\partial t} f_{t}^{-1}\right)\right]-P^{\prime \prime}\left(\nabla^{2} ;\left[\nabla, \nabla^{2}\right], \frac{\partial f_{t}}{\partial t} f_{t}^{-1}\right),
\end{aligned}
$$

where $P^{\prime \prime}(A ; B, C)$ is the second differential of $P$ at the matrix $A$ evaluated on $B$ and $C$ (this equals $\left.\partial^{2} / \partial b \partial c P^{\prime \prime}(A+b B+c C)_{\mid b=c=0}\right)$. Obviously $\left[\nabla, \nabla^{2}\right]$ vanishes (this is the second Bianchi identity) so that the above form is exact and the class of $\bar{P}\left(f_{0}^{*} \nabla, f_{1}^{*} \nabla\right)$ in $\wedge(X) / \mathrm{d} \wedge(X)$ vanishes.

Proposition 3.2. - If $\nabla_{0}^{E}, \nabla_{1}^{E}$ and $\nabla_{0}^{F}, \nabla_{1}^{F}$ are connexions on $E$ and $F$ respectively, if $P$ is an additive polynomial and ch is the Chern character, then for the obvious connexions on $E \oplus F$ and $E \otimes F$ one has in $\wedge(X) / \mathrm{d} \wedge(X)$ :

$$
\begin{aligned}
& \bar{P}\left(\nabla_{0}^{E} \oplus \nabla_{0}^{F}, \nabla_{1}^{E} \oplus \nabla_{1}^{F}\right)=\bar{P}\left(\nabla_{0}^{E}, \nabla_{1}^{E}\right)+\bar{P}\left(\nabla_{0}^{F}, \nabla_{1}^{F}\right), \\
& \overline{\operatorname{ch}}\left(\nabla_{0}^{E} \otimes \operatorname{Id}_{F}+\operatorname{Id}_{E} \otimes \nabla_{0}^{F}, \nabla_{1}^{E} \otimes \operatorname{Id}_{F}+\operatorname{Id}_{E} \otimes \nabla_{1}^{F}\right) \\
& \quad=\overline{\operatorname{ch}}\left(\nabla_{0}^{E}, \nabla_{1}^{E}\right) \wedge \operatorname{ch}\left(\nabla_{0}^{F 2}\right)+\operatorname{ch}\left(\nabla_{1}^{E 2}\right) \wedge \overline{\operatorname{ch}}\left(\nabla_{0}^{F}, \nabla_{1}^{F}\right) \\
& \quad=\operatorname{ch}\left(\nabla_{0}^{E 2}\right) \wedge \overline{\operatorname{ch}}\left(\nabla_{0}^{F}, \nabla_{1}^{F}\right)+\overline{\operatorname{ch}}\left(\nabla_{0}^{E}, \nabla_{1}^{E}\right) \wedge \operatorname{ch}\left(\nabla_{1}^{F 2}\right) .
\end{aligned}
$$

For a multiplicative invariant polynomial (or power series) such that $P(0)=1$ (as the total Chern class $c_{\text {tot }}$ or the $\widehat{A}$ or Todd genus) the theory can be slightly modified as follows: $P$ can be inverted in power series, let $P^{-1}$ be its inverse. For two connexions $\nabla_{0}$ and $\nabla_{1}$ on the same bundle $E$ over $X$, put

$$
\widehat{P}\left(\nabla_{0}, \nabla_{1}\right):=\bar{P}\left(\nabla_{0}, \nabla_{1}\right) \wedge P^{-1}\left(\nabla_{0}^{2}\right) .
$$

BULLETIN DE LA SOCIÉTÉ MATHÉMATIQUE DE FRANCE 
This form provides the following "multiplicative" transgression

$$
P\left(\nabla_{1}^{2}\right) \wedge P^{-1}\left(\nabla_{0}^{2}\right)=1+\mathrm{d} \widehat{P}\left(\nabla_{0} \nabla_{1}\right) .
$$

In view of Proposition 3.1, the class of $\widehat{P}$ in $\wedge(X) / \mathrm{d} \wedge(X)$ verifies

$$
\widehat{P}\left(\nabla_{0}, \nabla_{2}\right)=\widehat{P}\left(\nabla_{0}, \nabla_{1}\right)+\widehat{P}\left(\nabla_{1}, \nabla_{2}\right) \wedge\left(1+\mathrm{d} \widehat{P}\left(\nabla_{0}, \nabla_{1}\right)\right)
$$

for three connexions $\nabla_{0}, \nabla_{1}$ and $\nabla_{2}$ on the same bundle. In particular

$$
\widehat{P}\left(\nabla_{1}, \nabla_{0}\right)=\widehat{P^{-1}}\left(\nabla_{0}, \nabla_{1}\right)=-\widehat{P}\left(\nabla_{0}, \nabla_{1}\right) \wedge\left(1+\mathrm{d} \widehat{P}\left(\nabla_{0}, \nabla_{1}\right)\right)
$$

and for $\nabla_{0}^{E} \oplus \nabla_{0}^{F}$ and $\nabla_{1}^{E} \oplus \nabla_{1}^{F}$ on $E \oplus F$ the multiplicativity of $P$ gives

$$
\begin{aligned}
\widehat{P}\left(\nabla_{0}^{E}\right. & \left.\oplus \nabla_{0}^{F}, \nabla_{1}^{E} \oplus \nabla_{1}^{F}\right) \\
& =\widehat{P}\left(\nabla_{0}^{E}, \nabla_{1}^{E}\right)+\widehat{P}\left(\nabla_{0}^{F}, \nabla_{1}^{F}\right) \wedge\left(1+\mathrm{d} \widehat{P}\left(\nabla_{0}^{E}, \nabla_{1}^{E}\right)\right) \\
& =\widehat{P}\left(\nabla_{0}^{F}, \nabla_{1}^{F}\right)+\widehat{P}\left(\nabla_{0}^{E}, \nabla_{1}^{E}\right) \wedge\left(1+\mathrm{d} \widehat{P}\left(\nabla_{0}^{F}, \nabla_{1}^{F}\right)\right)
\end{aligned}
$$

((9) and $(10)$ are valid in $\wedge(X) / \mathrm{d} \wedge(X))$. This "multiplicative" transgression will not give anything exceptionnal in view of Proposition 3.6 but it will explain the reason for the appearance of some unusual additive characteristic polynomial in the sequel (see Lemma 4.1).

Chern-Simons transgression forms provide Chern-Weil type representatives for suspended characteristic classes on $K_{1}^{\text {top }}(X)$ : remember that $K_{1}^{\text {top }}(X)$ can be constructed as follows (see [27], Prop. 4.2 of Chap. II):

$$
K_{1}^{\mathrm{top}}(X)=\operatorname{Ker}\left(K_{0}^{\mathrm{top}}\left(S^{1} \times X\right) \rightarrow K_{0}^{\mathrm{top}}(\{\mathrm{pt}\} \times X)\right)
$$

where the map is the restriction to some point $\{\mathrm{pt}\}$ of $S^{1}$.

For any invariant polynomial $P$ the associated suspended class is defined as the composed map (see [27], §3.26 of Chap. V)

(11) $K_{1}^{\mathrm{top}}(X) \hookrightarrow K_{0}^{\mathrm{top}}\left(S^{1} \times X\right) \stackrel{[P]}{\longrightarrow} H^{\bullet}\left(S^{1} \times X\right) \rightarrow H^{1}\left(S^{1}\right) \times H^{\bullet}(X) \rightarrow H^{\bullet}(X)$

the second map being the usual characteristic class associated to $P$, the third one the projection of

$$
H^{\bullet}\left(S^{1} \times X\right) \cong H^{\bullet}\left(S^{1}\right) \otimes H^{\bullet}(X)=H^{0}\left(S^{1}\right) \otimes H^{\bullet}(X) \oplus H^{1}\left(S^{1}\right) \otimes H^{\bullet}(X)
$$

on the second factor and the last one is given by "integrating" along $S^{1}$ (or dividing by the canonical generator of $H^{1}\left(S^{1}, \mathbb{Z}\right)$ ).

For $\left(T_{N}, \alpha\right) \in K_{1}^{\text {top }}(X)$, consider the trivial rank $N$ bundle on $[0,1] \times X$ and identify its restrictions to $\{0\} \times X$ and $\{1\} \times X$ throw $\alpha$. This leads to a vector bundle $\xi_{\alpha}$ on $S^{1} \times X$. Consider the following connexion:

$$
\nabla=\mathrm{d} t \wedge \frac{\partial}{\partial t}+(1-t) \mathrm{d}+t \alpha^{*} \mathrm{~d}
$$

TOME $130-2002-\mathrm{N}^{\mathrm{O}} 2$ 
where $\mathrm{d}$ is the canonical connexion on $T_{N}$ over $X$. It fits to the identification defining $\xi_{\alpha}$ thus leading to a connexion on $\xi_{\alpha}$. Now in view of formulas (5), (11) and (12), for any invariant polynomial $P$ :

$$
S P\left(T_{N}, \alpha\right)=\int_{[0,1]} P\left(\nabla^{2}\right)=\bar{P}\left(\mathrm{~d}, \alpha^{*} \mathrm{~d}\right) .
$$

Note that the construction (11) is valid in integral cohomology if $P$ gives rise to an integral characteristic class (of course the above formula (13) only holds in real or complex cohomology). Note also that because $d$ is a flat connexion one has in this case (for a multiplicative $P$ with $P(0)=1$ ):

$$
\bar{P}\left(\mathrm{~d}, \alpha^{*} \mathrm{~d}\right)=\widehat{P}\left(\mathrm{~d}, \alpha^{*} \mathrm{~d}\right) .
$$

In fact any vector bundle $\xi$ on $S^{1} \times X$ defines a class in $K_{1}^{\text {top }}(X)$ as follows: let $p_{2}: S^{1} \times X \rightarrow X$ be the projection on the second factor and $\xi_{\mathrm{pt}}$ the restriction of $\xi$ to $\{\mathrm{pt}\} \times X$, the map $[\xi] \mapsto\left([\xi]-\left[p_{2}^{*} \xi_{\mathrm{pt}}\right]\right)$ defines a group epimorphism $K_{0}^{\text {top }}\left(S^{1} \times X\right) \rightarrow K_{1}^{\text {top }}(X)$. Take any connexion $\nabla$ on $\xi$, call $\nabla_{\mathrm{pt}}$ its restriction to $\xi_{\mathrm{pt}}$ and let $\alpha: \xi_{\mathrm{pt}} \rightarrow \xi_{\mathrm{pt}}$ be the $\mathcal{C}^{\infty}$ automorphism induced by parallel transport by $\nabla$ along $S^{1}$, then for an additive characteristic polynomial $P$, formula (13) remains valid (with $\nabla_{\mathrm{pt}}$ instead of $d$ ) whereas for a multiplicative one the right formula is

$$
S P(\xi)=S P\left(\xi_{\mathrm{pt}}, \alpha\right)=\left(\int_{S^{1}} P\left(\nabla^{2}\right)\right) \wedge P^{-1}\left(\nabla_{\mathrm{pt}}^{2}\right)=\widehat{P}\left(\nabla_{\mathrm{pt}}, \alpha^{*} \nabla_{\mathrm{pt}}\right) .
$$

3.2. The relevant cohomology groups. - For $X$ complex analytic and smooth let $\wedge^{k}(X)$ (resp. $\left.\wedge^{p, q}(X)\right)$ denote the space of $\mathcal{C}^{\infty}$ differential forms on $X$ of degree $k$ (resp. of complex type $(p, q)$ ). Remember the Hodge filtration of the de Rham complex provided by the

$$
F_{r}=\bigoplus_{\substack{p \geq r \\ 0 \leq q \leq \operatorname{dim} X}} \wedge^{p, q}(X) .
$$

The intersection $F_{p} \cap \wedge^{q}(X)$ will be denoted by $F_{p} \wedge^{q}(X)$. The $F_{r}$ are respected by the exterior differential $d$ so that one can consider for any $r$ and $s$ the cohomology groups $H^{s}\left(\wedge^{\bullet}(X) / F_{r}, \mathrm{~d}\right)$. The space $\bigoplus_{r=1}^{\operatorname{dim} X} H^{2 r-1}\left(\wedge(X) / F_{r}, \mathrm{~d}\right)$ will be denoted $H^{2 \bullet-1}\left(\wedge(X) / F_{\bullet}\right)$. This space is endowed with the two following group structures:

$$
\left\{\begin{array}{l}
(\alpha, \beta) \longmapsto \alpha+\beta, \\
(\alpha, \beta) \longmapsto \alpha+\beta \wedge(1+\mathrm{d} \alpha)=\beta+\alpha \wedge(1+\mathrm{d} \beta) .
\end{array}\right.
$$

If in addition $X$ is compact Kähler, then by Hodge theory

$$
H^{2 \bullet-1}\left(\wedge(X) / F_{\bullet}\right)=\bigoplus_{\substack{q \geq p \\ p+q \text { odd }}} H^{p, q}(X)
$$

BULletin DE LA SOCIÉtÉ MATHÉMATIQUE DE FRANCE 
so that any class can be represented by a closed form. Consequently the two above group structures (16) coïncide in this case.

Consider now a quasiprojective manifold $X$ and $\bar{X}$ some smooth projective compactification of $X$ such that $D=\bar{X} \backslash X$ is a normal crossing divisor. Such an $\bar{X}$ will be called a good compactification of $X$. Consider some neighbourhood $N$ of some point $p \in D$ modeled on a punctured polycylinder: the coordinates $\left(z_{i}\right)_{1 \leq i \leq d}$ are defining functions of the $d$ components of $D$ meeting at $p$. A logarithmic $\overline{\mathcal{C}}^{\infty}$ differential form on $\bar{X}$ is a $\mathcal{C}^{\infty}$ form on $X$ whose restriction to $N$ looks like:

$$
\sum_{0 \leq \ell \leq d} \sum_{i_{1}, i_{2}, \ldots, i_{\ell} \in\{1,2, \ldots, d\}} \alpha_{i_{1}, i_{2}, \ldots, i_{\ell}} \frac{\mathrm{d} z_{i_{1}}}{z_{i_{1}}} \wedge \frac{\mathrm{d} z_{i_{2}}}{z_{i_{2}}} \wedge \cdots \wedge \frac{\mathrm{d} z_{i_{\ell}}}{z_{i_{\ell}}}
$$

where the $\alpha_{i_{1}, i_{2}, \ldots, i_{\ell}}$ are $\mathcal{C}^{\infty}$ differential forms on $N$. The Hodge filtration of the space of logarithmic $\mathcal{C}^{\infty}$ forms on $\bar{X}$ is as above provided by the subspaces $F_{r L}(\bar{X})$ of logarithmic forms of complex types $(p, q)$ for $p \geq r$.

Denote by $\wedge_{L}(X)\left(\right.$ resp. $\wedge_{L}^{k}(X)$ resp. $\wedge_{L}^{p, q}(X)$ resp. $\left.F_{r L}\right)$ the inductive limit taken on good compactifications of $X$ of the spaces of logarithmic $\mathcal{C}^{\infty}$ forms (resp. of degree $k$ ones resp. of complex type $(p, q)$ ones resp. of the $F_{r L}(\bar{X})$ ). The exterior differential $d$ respects the $F_{r L}$ and one can as above consider the spaces $H^{s}\left(\wedge_{L}(X) / F_{r L}, d\right)$ and $H^{2 \bullet-1}\left(\wedge_{L}(X) / F_{\bullet L}\right)$ with group structures (16).

The complex $\left(\wedge_{L}(X), d\right)$ provides a resolution of the complex $\left(\Omega^{\bullet}(X), \partial\right)$ of holomorphic logarithmic forms $\left(\Omega^{p}(X)\right.$ is the space of $\bar{\partial}$-closed logarithmic forms of complex type $(p, 0))$. Thus Deligne's study of the mixed Hodge structure on the cohomology of quasiprojective manifolds [14], §3, yields that

$$
H^{k}(X, \mathbb{C})=H^{k}\left(\wedge_{L}(X), \mathrm{d}\right)
$$

carries for any $k$ a pure Hodge structure of weight $k$ (as in the compact Kähler case). Thus, the Fröhlicher spectral sequence degenerates at $E_{1}$ (see [14], lemme 3.2.6), any class in $H^{2 \bullet-1}\left(\wedge_{L}(X) / F_{\bullet L}\right)$ can be represented by a closed form and the two group structures (16) coïncide.

Finally consider the following situation: let $E$ be some algebraic vector bundle on $X, \mathbb{P}(E)$ its projectivisation and $\pi: \mathbb{P}(E) \rightarrow X$ the projection:

Proposition 3.3. - For any $r$ and $s$,

$$
\pi^{*}: H^{s}\left(\wedge_{L}(X) / F_{r L}\right) \longrightarrow H^{s}\left(\wedge_{L}(\mathbb{P}(E)) / F_{r L}\right)
$$

is an embedding.

Proof. - $\pi^{*}: H^{s}(X, \mathbb{C}) \rightarrow H^{s}(\mathbb{P}(E), \mathbb{C})$ is an embedding. Now from [14], Thm. 3.2.5 (iii), $\pi^{*}$ respects the Hodge filtration of the cohomology and thus also the quotients by $F_{r L}$ : the above map is well defined. Moreover from [14], Thm. 1.2.10 (iii), $\pi^{*}$ respects strictly the filtration which means that $\pi^{*}$ is an isomorphism of filtered objects from the coïmage of $\pi^{*}$ to its image (coïmage

TOME $130-2002-\mathrm{N}^{\mathrm{O}} 2$ 
here means quotient of the source space by the kernel of the morphism). This with the injectivity in complex cohomology proves the proposition.

3.3. The classes. - If $X$ is complex analytic, for any $(E, F, f) \in K_{0}^{\mathrm{rel}}(X)$, choose connexions $\nabla_{E}$ and $\nabla_{F}$ on $E$ and $F$ which are compatible with the holomorphic structures of $E$ and $F$. Such connexions will be hereafter simply called "compatible" if there is no ambiguity about the holomorphic structure. Their curvatures $\nabla_{E}^{2}$ and $\nabla_{F}^{2}$ are then of complex types $(2,0)+(1,1)$, so that for any invariant polynomial $P$ the $2 r$-degree parts of $P\left(\nabla_{E}^{2}\right)$ and $P\left(\nabla_{F}^{2}\right)$ lie in $F_{r} \wedge^{2 r}(X)$. Let $f^{*} \nabla_{F}$ be the pull-back connexion on $E$, then

$$
\mathrm{d} \bar{P}\left(\nabla_{E}, f^{*} \nabla_{F}\right)=P\left(\left(f^{*} \nabla_{F}\right)^{2}\right)-P\left(\nabla_{E}^{2}\right)=P\left(\nabla_{F}^{2}\right)-P\left(\nabla_{E}^{2}\right)
$$

lies in $\bigoplus_{r} F_{r} \wedge^{2 r}(X)$.

Definition. - The (Nadel) characteristic class $\mathcal{N}_{P}(E, F, f)$ is defined to be the class of $\bar{P}\left(\nabla_{E}, f^{*} \nabla_{F}\right)$ in the space $H^{2 \bullet-1}\left(\wedge(X) / F_{\bullet}\right)$ for any compatible connexions $\nabla_{E}$ and $\nabla_{F}$.

If $P$ is multiplicative such that $P(0)=1$, the (Nadel) "multiplicative" characteristic class $\mathcal{M}_{P}(E, F, f)$ is defined to be the class of $\widehat{P}\left(\nabla_{E}, f^{*} \nabla_{F}\right)$ in the space $H^{2 \bullet-1}\left(\wedge(X) / F_{\bullet}\right)$ for any compatible connexions $\nabla_{E}$ and $\nabla_{F}$.

If $X$ is quasiprojective, (17) implies (in particular) that any logarithmic exact form is the coboundary of some logarithmic form, and thus the injectivity of the following map:

$$
\wedge_{L}(X) / \mathrm{d} \wedge_{L}(X) \longrightarrow \wedge(X) / \mathrm{d} \wedge(X)
$$

Moreover if $\alpha \in \wedge(X)$ verifies that $\mathrm{d} \alpha$ is logarithmic, then there exists some $\beta \in \wedge_{L}(X)$ such that $\alpha-\beta$ is closed and hence (because of (17)) cohomologous to some closed logarithmic form $\gamma$. Thus, $\gamma+\beta \in \wedge_{L}(X)$ and $\alpha-(\gamma+\beta)$ is exact, so that $\alpha$ lies in the image of the above map (18).

Any algebraic vector bundle $E$ on $X$ is the restriction to $X$ of some algebraic vector bundle $\bar{E}$ on some compactification $\bar{X}$ of $X$. A connexion on $E$ will be said to be compatible with the algebraic structure of $E$ (or simply "compatible") if it is the restriction to $E$ of a compatible connexion on some such extension $\bar{E}$ of $E$ (note that this choice is noncanonical in the sense that one could allow more general connexions namely logarithmic ones or with controlled curvature bounds ones from which the algebraic structure of $E$ can be unambiguously recovered). Now $\bar{P}\left(\nabla_{E}, f^{*} \nabla_{F}\right)$ has no reason to be a logarithmic form even for compatible connexions $\nabla_{E}$ and $\nabla_{F}$. But $\mathrm{d} \bar{P}\left(\nabla_{E}, f^{*} \nabla_{F}\right)$ is exact and obviously logarithmic, this yields that $\bar{P}\left(\nabla_{E}, f^{*} \nabla_{F}\right)$ lies in the image of the map (18).

Let $\overline{P L}\left(\nabla_{E}, f^{*} \nabla_{F}\right)$ be its pre-image in $\wedge_{L}(X) / \mathrm{d} \wedge_{L}(X)$.

If $P$ is multiplicative with $P(0)=1$, then $\widehat{P}\left(\nabla_{E}, f^{*} \nabla_{F}\right)$ also lies in the image of the map (18): let $\widehat{P L}\left(\nabla_{E}, f^{*} \nabla_{F}\right)$ be its pre-image in $\wedge_{L}(X) / \mathrm{d} \wedge_{L}(X)$. Then 
of course

$$
\widehat{P L}\left(\nabla_{E}, f^{*} \nabla_{F}\right)=\overline{P L}\left(\nabla_{E}, f^{*} \nabla_{F}\right) \wedge P^{-1}\left(\nabla_{E}^{2}\right),
$$

and it is straightforward that $\overline{P L}\left(\nabla_{E}, f^{*} \nabla_{F}\right)$ and $\widehat{P L}\left(\nabla_{E}, f^{*} \nabla_{F}\right)$ satisfy the same properties as stated in Propositions 3.1 and 3.2 and (8), (9) and (10).

Definition 3.4. - The (Nadel) characteristic class $\mathcal{N}_{P}(E, F, f)$ is defined to be the class of $\overline{P L}\left(\nabla_{E}, f^{*} \nabla_{F}\right)$ in the space $H^{2 \bullet-1}\left(\wedge_{L}(X) / F_{\bullet L}\right)$ for any compatible connexions $\nabla_{E}$ and $\nabla_{F}$.

If $P$ is multiplicative such that $P(0)=1$, the (Nadel) "multiplicative" characteristic class $\mathcal{M}_{P}(E, F, f)$ is defined to be the class of $\widehat{P L}\left(\nabla_{E}, f^{*} \nabla_{F}\right)$ in the space $H^{2 \bullet-1}\left(\wedge_{L}(X) / F_{\bullet L}\right)$ for any compatible connexions $\nabla_{E}$ and $\nabla_{F}$.

TheOREM 3.5. - $(\alpha)$ These classes do not depend on the chosen compatible connexions $\nabla_{E}$ and $\nabla_{F}$.

$(\beta)$ They vanish if $f$ is an holomorphic (resp. algebraic) isomorphism.

$(\gamma)$ They are invariant throw isotopy of $f$.

$(\delta) \mathcal{N}_{P}\left(E, E^{\prime} \oplus E^{\prime \prime}, s \oplus p\right)=0$ for any exact sequence

$$
0 \rightarrow E^{\prime} \stackrel{i}{\longrightarrow} E \stackrel{p}{\longrightarrow} E^{\prime \prime} \rightarrow 0
$$

and any $\mathcal{C}^{\infty}$ left inverse $s: E \rightarrow E^{\prime}$ of $i$ (and the same holds for the class $\left.\mathcal{M}_{P}\left(E, E^{\prime} \oplus E^{\prime \prime}, s \oplus p\right)\right)$.

Corollary. - If $P$ is an additive polynomial, then $\mathcal{N}_{P}$ provides a morphism

$$
\mathcal{N}_{P}: K_{0}^{\mathrm{rel}}(X) \longrightarrow H^{2 \bullet-1}\left(\wedge_{(L)}(X) / F_{\bullet}(L)\right)
$$

for the usual addition on the latter space.

If $P$ is multiplicative with $P(0)=1, \mathcal{M}_{P}$ is a group morphism for the other group structure on $H^{2 \bullet-1}\left(\wedge_{(L)}(X) / F_{\bullet}(L)\right)(c f$. $(16))$.

REMARK. - Properties analogous to $(\alpha),(\gamma)$ and $(\delta)$ are partially proved in [29], $\S 6.4$ (see also [11], Lemma 2.3 and note that it is not necessary to require that $\nabla_{E}$ and $\nabla_{F}$ be compatible with any hermitian metric on $E$ nor on $F$ ), [35], §5, [30], §§2.8 and 2.10, and [29], §6.7.

Proof. - The corollary is an easy consequence of the theorem, Proposition 3.2 and the linearity of the map which associates $\overline{P L}$ to $\bar{P}$ if $X$ is quasiprojective. The properties for $\mathcal{M}_{P}$ are also easily deduced from corresponding ones for $\mathcal{N}_{P}$.

$(\delta)$ : If $X$ is complex analytic, let $j: E^{\prime \prime} \rightarrow E$ be any $\mathcal{C}^{\infty}$ map such that

$$
p \circ j=\operatorname{Id}_{E^{\prime \prime}} \text { and } s \circ j=0 .
$$

Consider the projectors in $E$ :

$$
\pi^{\prime}=i \circ s \quad \text { and } \quad \pi^{\prime \prime}=j \circ p .
$$

TOME $130-2002-\mathrm{N}^{\mathrm{O}} 2$ 
If $\nabla_{E}$ is a connexion on $E$ compatible with its holomorphic structure, then

$$
\nabla_{E^{\prime}}=s \circ \nabla_{E} \circ i \text { and } \nabla_{E^{\prime \prime}}=p \circ \nabla_{E} \circ j
$$

are compatible with the holomorphic structures of $E^{\prime}$ and $E^{\prime \prime}$. Put

$$
\nabla_{t}=\pi^{\prime} \nabla_{E} \pi^{\prime}+\pi^{\prime \prime} \nabla_{E} \pi^{\prime \prime}+(1-t) \pi^{\prime} \nabla_{E} \pi^{\prime \prime} .
$$

It is then easily verified that

$$
\nabla_{1}=(s \oplus p)^{*}\left(\nabla_{E^{\prime}} \oplus \nabla_{E^{\prime \prime}}\right)
$$

that $\nabla_{0}$ is as $\nabla_{E}$ compatible with the holomorphic structure of $E$, and that

$$
P\left(\nabla_{t}^{2}+b \frac{\partial \nabla_{t}}{\partial t}\right)=P\left(\nabla_{t}^{2}\right)=P\left(\nabla_{E^{\prime}}^{2}\right)+P\left(\nabla_{E^{\prime \prime}}^{2}\right)
$$

is constant with respect to $b$, which shows that $\bar{P}\left(\nabla_{0}, \nabla_{1}\right)$ vanishes $(c f$. (4)).

If now $X$ is quasiprojective, any such algebraic exact sequence on $X$ is the restriction of some exact sequence

$$
0 \rightarrow \bar{E}^{\prime} \longrightarrow \bar{E} \longrightarrow \bar{E}^{\prime \prime} \rightarrow 0
$$

on some compactification of $X$. The same argument yields the answer because $\nabla_{0}$ and $\nabla_{1}$ are obviously compatible with the algebraic structures of $E$ and $E^{\prime} \oplus E^{\prime \prime}$ on $X$.

If $X$ is complex analytic, $(\alpha)$ is in view of formula (6) equivalent to saying that $\bar{P}\left(\nabla_{1}^{E}, \nabla_{2}^{E}\right)$ has vanishing class in $H^{2 \bullet-1}\left(\wedge(X) / F_{\bullet}\right)$ if $\nabla_{1}^{E}$ and $\nabla_{2}^{E}$ are compatible with the same holomorphic structure of $E$. But this is a consequence of a simple study of complex types in formula (5) applied to two connexions compatible with the same holomorphic structure on the same bundle.

However if $X$ is quasiprojective, two extensions of $E$ can be lifted to the same compactification $\bar{X}$ of $X$, but they need not be isomorphic on $\bar{X}$. If $E$ is a line bundle, two extensions on the same $\bar{X}$ are meromorphically isomorphic, and the difference of two compatible connexions is easily seen to be a $(1,0)$ type exact logarithmic form on $X$. Therefore the corresponding $\overline{P L}$ classes vanish. In the case where $E$ is a direct sum of line bundles, suppose $E_{1}$ and $E_{2}$ are two extensions of $E$ to some compactification $\bar{X}$ of $X$ which respects this decomposition: if $P$ is additive, the result directly follows from Proposition 3.2 and the case of line bundles; now any invariant polynomial $P$ is obtained by sums and exterior products of additive ones (the homogeneous components of the Chern character $\operatorname{tr} \exp (i / 2 \pi \bullet))$ so that $(7)$ and the obvious relation

$$
\overline{P+Q}\left(\nabla_{0}, \nabla_{1}\right)=\bar{P}\left(\nabla_{0}, \nabla_{1}\right)+\bar{Q}\left(\nabla_{0}, \nabla_{1}\right)
$$

yield the result.

BULletin DE LA SOCiÉtÉ MATHÉMATIQUE DE FRANCE 
Finally for general $E$, one can use the trick by Zucker and Kashiwara [39], p. 408. Consider the projective flag bundle $\mathbb{P F}(E)$ (see [23], p. 138). It is obtained by successive projectivisation of vector bundles (and it is still a quasiprojective manifold) so that the functoriality of $\overline{P L}$ and Proposition 3.3 reduces the case of $E$ to the case of $\pi^{*} E$ on $\mathbb{P F}(E)$, where $\pi^{*} E$ is the pullback of $E$ by the projection $\pi: \mathbb{P F}(E) \rightarrow X . \pi^{*} E$ is filtered on $\mathbb{P F}(E)$,

$$
0=F_{0} \subset F_{1} \subset \cdots \subset F_{\mathrm{rkE}}=\pi^{*} E,
$$

so that for any $i$ the quotient $F_{i} / F_{i-1}$ is an algebraic line bundle. Thus repeated use of property $(\delta)$ reduces the case of $E$ to the case of a direct sum of line bundles. (I don't know if it is possible to find two compatible connexions on $E_{1}$ and $E_{2}$ such that the associated Chern-Simons form is directly logarithmic.)

$(\beta)$ is a straightforward consequence of $(\alpha)$.

$(\gamma)$ is a straightforward consequence of Proposition 3.1.

Proposition 3.6. - If $X$ is compact Kähler or $X$ is quasiprojective, then for any multiplicative polynomial $P$ such that $P(0)=1$ the following maps coïncide:

$$
\mathcal{M}_{P}=\mathcal{N}_{\log P}: K_{0}^{\mathrm{rel}}(X) \longrightarrow H^{2 \bullet-1}\left(\wedge_{(L)}(X) / F_{\bullet}(L)\right) .
$$

Proof. — The point is that classes in $H^{2 \bullet-1}\left(\wedge_{(L)}(X) / F_{\bullet}(L)\right)$ can be represented by closed forms. So if $Q$ and $R$ are invariant polynomials and $\nabla_{0}$ and $\nabla_{1}$ are connexions on $E$ whose curvature forms are of complex types $(2,0)+(1,1)$, then in $H^{2 \bullet-1}\left(\wedge_{(L)}(X) / F_{\bullet}(L)\right), \bar{Q}\left(\nabla_{0}, \nabla_{1}\right) \wedge R\left(\nabla_{1}^{2}\right)$ and $\bar{Q}\left(\nabla_{0}, \nabla_{1}\right) \wedge R\left(\nabla_{0}^{2}\right)$ have the same image. In particular from Property $(7), m \bar{Q}\left(\nabla_{0}, \nabla_{1}\right) \wedge Q^{\wedge m-1}\left(\nabla_{0}^{2}\right)$ and $\overline{Q^{\wedge m}}\left(\nabla_{0}, \nabla_{1}\right)$ have same image in $H^{2 \bullet-1}\left(\wedge_{(L)}(X) / F_{\bullet}(L)\right)$. If one sets $P=1+Q$, one gets that

$$
\overline{\log P}\left(\nabla_{0}, \nabla_{1}\right)=\sum_{i=1}^{\infty} \frac{(-1)^{i-1}}{i} \overline{Q^{\wedge i}}\left(\nabla_{0}, \nabla_{1}\right)
$$

has same image in $H^{2 \bullet-1}\left(\wedge_{(L)}(X) / F_{\bullet}(L)\right)$ as

$$
\sum_{i=1}^{\infty} \bar{Q}\left(\nabla_{0}, \nabla_{1}\right) \wedge(-1)^{i-1} Q^{\wedge i-1}\left(\nabla_{0}^{2}\right)=\bar{P}\left(\nabla_{0}, \nabla_{1}\right) \wedge P^{-1}\left(\nabla_{0}^{2}\right)
$$

which proves the statement.

\section{Nadel's conjecture and its proof}

This section is organised as follows. In 4.1 Nadel's original conjecture is shown to be a consequence of Theorem 1.1. Some facts about Hodge-Deligne and Deligne-Beilinson cohomology are then recalled in 4.2, and the proof of Theorem 1.1 is reduced to the study of the compatibility of $\mathcal{M}_{c_{\text {tot }}}$ with Chern classes in Hodge-Deligne or Deligne-Beilinson cohomology. This is proved in 4.3 and

TOME $130-2002-\mathrm{N}^{\mathrm{O}} 2$ 
relies heavily on Brylinski and Zucker's works [11], [39] about compatibility of Chern-Cheeger-Simons and Hodge-Deligne-Beilinson classes. The link with Karoubi's multiplicative $K$-theory [28], [29], [30] is also explained.

4.1. Nadel's conjecture. - Note first that if $P$ is a multiplicative polynomial such that $P(0)=1$ then the suspended classes $S P$ and $S \log P$ coïncide on $K_{1}^{\text {top }}(X)$ as can be seen by the preceding calculation (Prop. 3.6) performed with flat connexions d and $\alpha^{*} \mathrm{~d}$ (in the notation of (12), (13) and (14)).

Let $c_{\text {tot }}=1+\sum_{r=1}^{\infty} c_{r}$ be the total Chern class and $S c_{\text {tot }}$ be its suspension to $K_{1}^{\text {top }}(X)$. Consider the Chern character ch and its $2 r$-degree homogeneous part:

$$
c_{\text {tot }}=\operatorname{det}\left(\operatorname{Id}+\frac{i}{2 \pi} \cdot\right), \quad \operatorname{ch}=\operatorname{tr} \exp \left(\frac{i}{2 \pi} \cdot\right), \quad \operatorname{ch}_{r}=\operatorname{tr} \frac{1}{r !}\left(\frac{i}{2 \pi} \cdot\right)^{r}
$$

and define the "Renormalized" Chern character to be:

$$
R \operatorname{ch}=\sum_{r=1}^{\infty}(-1)^{r-1}(r-1) ! \operatorname{ch}_{r}=\operatorname{tr} \sum_{r=1}^{\infty} \frac{(-1)^{r-1}}{r}\left(\frac{i}{2 \pi} \cdot\right)^{r} .
$$

Lemma 4.1. $-R \mathrm{ch}=\log c_{\mathrm{tot}}$.

Proof. - Consider the formal power series

$$
N(\theta)=\sum_{r \geq 1}\left(r ! \operatorname{ch}_{r}\right) \theta^{r} \quad \text { and } \quad c_{\text {tot }}(\theta)=\sum_{i \in \mathbb{N}} c_{i} \theta^{i} .
$$

Newton's formulas read

$$
\begin{array}{r}
\left(r ! \mathrm{ch}_{r}\right)=\sum_{j=1}^{r-1}(-1)^{r-1-j}\left(j ! \mathrm{ch}_{j}\right) \cap c_{r-j}+(-1)^{r-1} r c_{r} \\
\Longleftrightarrow N(-\theta) \cap c_{\mathrm{tot}}(\theta)=-\theta c_{\mathrm{tot}}^{\prime}(\theta)
\end{array}
$$

where $c_{\mathrm{tot}}^{\prime}(\theta)$ is the derived power series of $c_{\mathrm{tot}}(\theta)$. Now the series

$$
R \operatorname{ch}(\theta)=\sum_{r \geq 1}(-1)^{r-1}(r-1) ! \operatorname{ch}_{r} \theta^{r}
$$

is a primitive of

$$
\frac{-1}{\theta} N(-\theta)=c_{\text {tot }}^{\prime}(\theta) \cap c_{\text {tot }}^{-1}(\theta)
$$

where $c_{\mathrm{tot}}^{-1}(\theta)$ is the inverse power series of $c_{\mathrm{tot}}(\theta)$. This yields the result.

Consider the obvious morphism $\psi: H^{\text {odd }}(X, \mathbb{Z}) \rightarrow H^{2 \bullet-1}\left(\wedge_{(L)}(X) / F_{\bullet}(L)\right)$. BULLETIN DE LA SOCIÉtÉ MATHÉMATIQUE DE FRANCE 
LEMMA 4.2. - The following diagram commutes

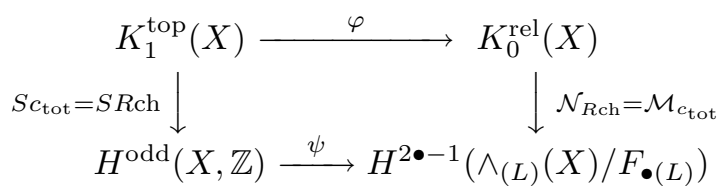

(for $X$ compact Kähler or quasiprojective).

Proof. - Rch is as the Chern character an additive polynomial so that the "right vertical" map is well defined. If $P$ is any integral additive invariant polynomial, one obtains from formulas (13) and (5)

$$
\psi\left(S P\left(T_{N}, \alpha\right)\right)=\mathcal{N}_{P}\left(\varphi\left(T_{N}, \alpha\right)\right) \in H^{2 \bullet-1}\left(\wedge_{(L)}(X) / F_{\bullet}(L)\right) .
$$

This proves the lemma.

Note that in the complex analytic case, one gets two commutative diagrams with same lines and same left column but which need not coïncide in general.

Corollary. - If $X$ is compact Kähler or quasiprojective, $\mathcal{M}_{c_{\text {tot }}}$ provides a group morphism

$$
\mathcal{M}_{c_{\text {tot }}}: K_{0}^{\text {hom }}(X) \longrightarrow \bigoplus_{r=1}^{\operatorname{dim} X} J_{r}(X)
$$

with values in Griffiths' intermediate jacobians

$$
J_{r}(X)=H^{2 r-1}\left(\wedge_{(L)}^{\bullet}(X) / F_{r(L)}\right) / \psi\left(H^{2 r-1}(X, \mathbb{Z})\right) .
$$

Note that the compact-Kähler or quasiprojective condition is not necessary, but the group structure on $\bigoplus_{r} J_{r}(X)$ is then much less obvious.

The link between Theorem 1.1 and Nadel's original conjecture follows the LemMA 4.3. - For $X$ compact complex analytic consider the natural map

$$
\pi: H^{2 \bullet-1}\left(\wedge(X) / F_{\bullet}\right) \longrightarrow \bigoplus_{r=1}^{\left[\frac{1}{2}(\operatorname{dim} X+1)\right]} H^{2 r-1}\left(\wedge(X) / F_{2 r-2}\right) \cong H^{\text {odd }}(X, \mathcal{O})
$$

then for any $r$ the class $\mathcal{E}^{2 r-1}$ of [35], Def. p. 40, coïncides with $\pi \circ \mathcal{N}_{r ! \mathrm{ch}_{r}}$

(Here as above, $\mathrm{ch}_{r}$ is the $2 r$-degree homogeneous part of the Chern character.)

Proof. - Let $\bar{\partial}_{E}$ and $\bar{\partial}_{F}$ be the Cauchy-Riemann operators corresponding to the holomorphic structures of $E$ and $F$, let $\nabla_{E}$ and $\nabla_{F}$ be compatible connexions, and consider the homotopy

$$
\nabla_{t}=(1-t) \nabla_{E}+t f^{*} \nabla_{F}
$$

TOME $130-2002-\mathrm{N}^{\mathrm{O}} 2$ 
then the (0,2)-type part of $\nabla_{t}^{2}$ is $-t(1-t)\left(f^{*} \bar{\partial}_{F}-\bar{\partial}_{E}\right)^{2}$. Using formula (5), one obtains that the $(0,2 r-1)$-type part of $r ! \overline{c h}_{r}\left(\nabla_{E}, f^{*} \nabla_{F}\right)$ is:

$$
(-1)^{r-1} r\left(\frac{i}{2 \pi}\right)^{r}\left(\int_{0}^{1}(t(1-t))^{r-1} \mathrm{~d} t\right) \operatorname{tr}\left[\left(f^{*} \bar{\partial}_{F}-\bar{\partial}_{E}\right)^{2 r-1}\right] .
$$

The integral equals $(r-1) !^{2} /(2 r-1)$ ! and this gives Nadel's formula [35], p. 40 .

Corollary. - Nadel's original conjecture holds.

Proof. - Nadel's conjecture is nothing but the statement of Theorem 1.1 after projection by $\pi$ for "the Chern character without denominators", namely $\mathcal{N}_{r ! \mathrm{ch}_{r}}$ for all $r$. Of course each homogeneous component of this class is an integral multiple of the corresponding homogeneous component of $\mathcal{N}_{R \mathrm{ch}}\left(=\mathcal{M}_{c_{\mathrm{tot}}}, X\right.$ being projective). Thus, Theorem 1.1 implies Nadel's conjecture.

REMARK. - In view of Lemma 4.3, the generalisation of Nadel's integrality result [35], §6, is provided by Lemma 4.2 (see also the example [35], §12)

REMARK 4.4. - It is possible to deduce the map $\mathcal{M}_{c_{\text {tot }}}$ from the Abel-Jacobi map, but the converse is not possible beyond the degree $r=2$ because the map $c_{\text {tot }}-1: K_{0}^{\text {hom }} \rightarrow \mathrm{CH}_{\text {hom }}$ need not be surjective. If $Z \in \mathrm{CH}_{\text {hom }}(X)$ is some cycle of codimension $m$, the element $[\xi] \in K_{0}^{\text {hom }}(X)$ obtained by alternating sum of the vector bundles entering a left resolution of the sheaf $\iota_{*} \mathcal{O}_{Z}$ (where $\iota: Z \rightarrow X$ is the inclusion), verifies that $\operatorname{ch}([\xi])-Z \in \mathrm{CH}_{\mathrm{hom}}(X) \otimes \mathbb{Q}$ is of codimension strictly greater than $m$, and $c_{m}([\xi])=(m-1) ! Z$ because of the vanishing of lower Chern classes of $[\xi]$. This $(m-1)$ ! is an obstruction to invert the relation between $\mathcal{M}_{c_{\text {tot }}}$ and $A J$. (See also Remark 6.9.)

4.2. Hodge-Deligne-Beilinson cohomology. - For any $j$, let $\Omega_{X}^{j}$ be the sheaf of germs of logarithmic holomorphic forms of degree $j$ on some good compactification $\bar{X}$ of $X$. The relevant Deligne-Beilinson cohomology groups in our context is the following (it is named "Hodge-Deligne" or "analytic Deligne" if $X$ is complex analytic, and constructed with the sheaves of not necessarily logarithmic holomorphic forms on $X)$ :

$$
H_{\mathcal{D}}^{2 p}(X, \mathbb{Z}(p))=\mathbb{H}^{2 p}\left(\bar{X}, \mathbb{Z} \stackrel{(2 \pi i)^{p}}{\longrightarrow} \mathcal{O}_{\bar{X}} \rightarrow \Omega_{X}^{1} \rightarrow \cdots \rightarrow \Omega_{X}^{p-1}\right)
$$

where $\mathbb{H}^{2 p}$ is the $2 p$-th hypercohomology group of the indicated complex of sheaves on $\bar{X}$ (see [17], [15]). It is shown to be independent on the choice of the good compactification $\bar{X}$ and it fits in the following exact sequence

$$
0 \rightarrow J_{p}(X) \stackrel{j}{\longrightarrow} H_{\mathcal{D}}^{2 p}(X, \mathbb{Z}(p)) \stackrel{\pi}{\longrightarrow} H^{p, p}(X, \mathbb{Z}) \rightarrow 0
$$

where $H^{p, p}(X, \mathbb{Z})$ is the group of degree $2 p$ integral cohomology classes whose image in $H^{2 p}(X, \mathbb{C})$ can be represented by logarithmic closed forms of complex type $(p, p)$. 
Deligne defined a cycle map (for projective $X$ ) (see [17], §7, [15])

$$
\mathrm{CH}^{p}(X) \stackrel{\tilde{\gamma}}{\longrightarrow} H_{\mathcal{D}}^{2 p}(X, \mathbb{Z}(p))
$$

from the full Chow group of $X$. This map fits in the commutative diagram

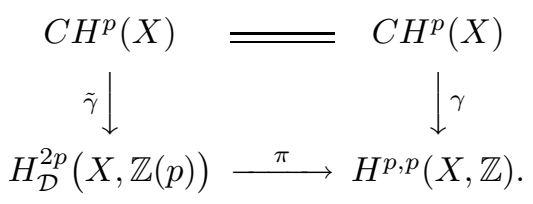

He also constructed Chern classes $\widetilde{c}_{r}$ for holomorphic vector bundles with values in Deligne-Beilinson cohomology which fit in the following commutative diagram (here $X$ need not be projective, see [11], [39] or [17], $\S 8$ ):

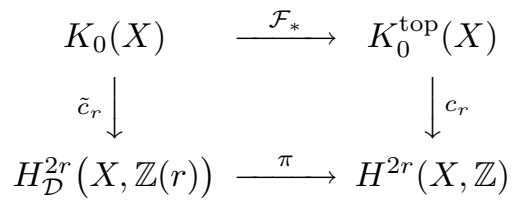

(the usual Chern class $c_{r}$ on $K_{0}^{\mathrm{top}}(X)$ need not have its values contained in $\left.H^{r, r}(X, \mathbb{Z})\right)$. Let $\widetilde{c}_{\text {tot }}$ be the total Chern class $1+\sum_{r=1}^{n} \widetilde{c}_{r}$.

Theorem 4.5. - If $X$ is projective, the following diagram commutes

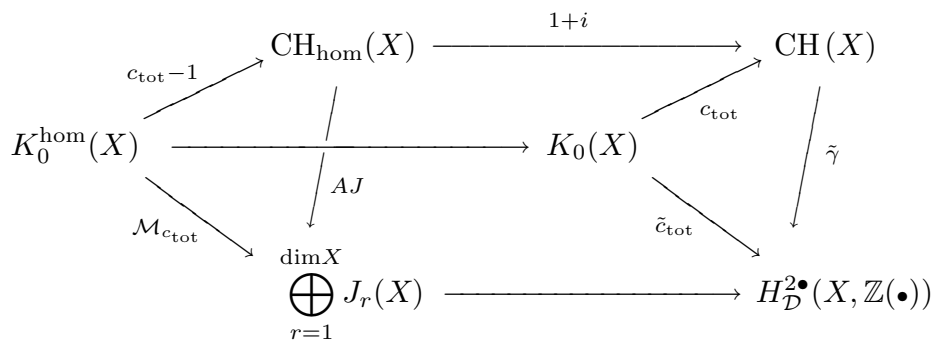

where $H_{\mathcal{D}}^{2 \bullet}(X, \mathbb{Z}(\bullet))$ is the sum $\bigoplus_{r=1}^{\operatorname{dim} X} H_{\mathcal{D}}^{2 r}(X, \mathbb{Z}(r))$.

(The maps are morphisms for the intersection '.' on $\mathrm{CH}(X)$, the multiplicative structure on $H_{\mathcal{D}}^{2 \bullet}(X, \mathbb{Z}(\bullet))$ (see [17], 33$), 1$ being the unit for these structures. The "product" on $\mathrm{CH}_{\text {hom }}(X)$ is $(x, y) \mapsto(1+x) \cdot(1+y)-1$ and $i$ is the canonical injection of $\mathrm{CH}_{\text {hom }}(X)$ into $\mathrm{CH}(X)$.)

Corollary. - Theorem 1.1 holds true.

Proof. - In fact the commutativity of the "left" triangle will be deduced from the commutativity of all the other faces of the "prism" and the fact that the three "horizontal" morphisms are all injective.

Commutativity of the "top" square is trivial. Note that by (20) and (21), $\widetilde{\gamma}$ gives rise to a map $\widetilde{\gamma}: \mathrm{CH}_{\mathrm{hom}}^{k}(X) \rightarrow J^{k}(X)$. That this map coïncides

TOME $130-2002-\mathrm{N}^{\mathrm{O}} 2$ 
with the Abel-Jacobi map is shown by Gillet in [19] (see also [17], §7.12, [25], Lemma 1.22, [21] or Thm. 3.5, [15]), so the "rear" square commutes.

That the "right" triangle commutes is a classical fact (see [17], §8).

The remaining of this section is devoted to proving the commutativity of the "front bottom" square.

TheOREM 4.6. - If $X$ is quasiprojective, the following diagram commutes:

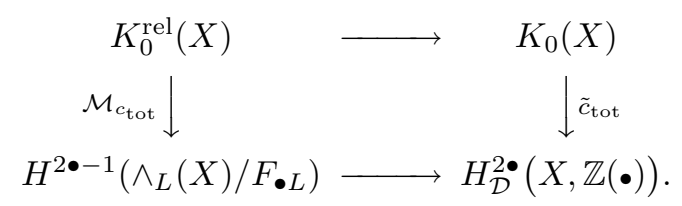

In fact the corresponding result will altogether be shown to hold for a complex analytic manifold $X$ and analytic Hodge-Deligne cohomology.

4.3. Proof of Theorem 4.6. - Let $\bar{X}$ be some good compactification of $X$ and denote by $A_{L}^{p, q}$ the sheaf on $\bar{X}$ of logarithmic forms of complex type $(p, q)$. The Dolbeault resolution

$$
0 \rightarrow \Omega_{X}^{p} \longrightarrow A_{L}^{p, 0} \stackrel{\bar{\partial}}{\longrightarrow} A_{L}^{p, 1} \stackrel{\bar{\partial}}{\longrightarrow} \cdots \stackrel{\bar{\partial}}{\longrightarrow} A_{L}^{p, n} \rightarrow 0
$$

provides that $H_{\mathcal{D}}^{2 k}(X, \mathbb{Z}(k))$ equals the following hypercohomology group:

$$
H_{\mathcal{D}}^{2 k}(X, \mathbb{Z}(k))=\mathbb{H}^{2 k}\left(\bar{X}, 0 \rightarrow \mathbb{Z} \stackrel{(2 \pi i)^{k}}{\longrightarrow} \bigoplus_{p \leq k} A_{L}^{p, q}\right) .
$$

(The operation is d in the complex and $\mathbb{Z}$ maps into $A_{L}^{0,0}$.)

Denote now by $A^{k}$ the sheaf on $X$ of $\mathcal{C}^{\infty}$ differential forms of degree $k$ (without logarithmic condition) and by $\widehat{H}^{2 r}(X, \mathbb{Z}(r))$ the $r$-th Cheeger-Simons' group of differential characters ( $c f$. reference [12], the notation being there $\left.\widehat{H}^{2 r-1}(X, \mathbb{C} / \mathbb{Z})\right)$. It follows from [39], (3.2.2), and [11], $\S 2$, that it is the $2 r$-th hypercohomology group of the complex

$$
0 \rightarrow \mathbb{Z} \stackrel{(2 \pi i)^{r}}{\longrightarrow} A^{<2 r}(X)
$$

where $A^{<2 r}(X)$ is the complex of sheaves of $\mathcal{C}^{\infty}$ differential forms on $X$ truncated in degree $\leq 2 r-1$, and $\mathbb{Z}$ maps into $A^{0}(X)$ (the sheaf of $\mathcal{C}^{\infty}$ functions). Let $\wedge_{0}(X)$ be the subspace of $\wedge(X)$ consisting of closed forms whose cohomology class is integral (exact forms are also included in $\wedge_{0}(X)$ ). Reference [12], Thm. 1.1 states that the group of differential characters fits in the following exact sequence:

$$
0 \rightarrow \wedge(X) / \wedge_{0}(X) \stackrel{j}{\longrightarrow} \widehat{H}^{2 \bullet}(X, \mathbb{Z}(\bullet)) \longrightarrow H^{\text {even }}(X, \mathbb{Z}) \rightarrow 0 .
$$

BULLETIN DE LA SOCiÉTÉ MATHÉMATIQUe DE FRANCE 
Let $K_{0}^{\nabla}(X)$ be the $K_{0}$-group of $\mathcal{C}^{\infty}$ vector bundles with connection (modulo exact sequences which respect connexions). Cheeger and Simons [12], Thm. 2.2, constructed characteristic classes for any invariant polynomial $P$

$$
\check{P}: K_{0}^{\nabla}(X) \longrightarrow \widehat{H}^{2 \bullet}(X, \mathbb{Z}(\bullet))
$$

using universal spaces for vector bundles with connexions. The link with ChernSimons transgression forms is that if $\nabla_{0}$ and $\nabla_{1}$ are connexions on the same bundle, then (cf. [12], Prop. 2.9 or [13], Prop. 3.8)

$$
\check{P}\left(\left[E, \nabla_{1}\right]\right)-\check{P}\left(\left[E, \nabla_{0}\right]\right)=j\left(\bar{P}\left(\nabla_{0}, \nabla_{1}\right)\right) \text {. }
$$

(In the notation of [12], (or [13], §2) $P$ is taken to be an homogeneous polynomial of degree $k$ on $\operatorname{GL}(n, \mathbb{C})^{k}$ applied to some homogeneous differential form with values in $\operatorname{GL}(n, \mathbb{C})^{k}:\left(\nabla^{2}\right)^{\wedge k}$ for the Chern-Weil form, and $\partial \nabla_{t} / \partial t \wedge$ $\left(\nabla_{t}^{2}\right)^{\wedge k-1}$ for the Chern-Simons form. Checking the compatibility of [12], Prop. 2.9 (or [13], Prop. 3.8) with (5) above is straightforward.)

Take the equation (24) for $P=c_{\text {tot }}$, multiply it by $\check{c}_{\text {tot }}^{-1}\left(E, \nabla_{0}\right)$ and add 1 . From [12], Eqn. (1.15) one obtains:

$(25) \check{c}_{\text {tot }}\left(\left[E, \nabla_{1}\right]-\left[E, \nabla_{0}\right]\right)=\check{c}_{\text {tot }}\left(E, \nabla_{1}\right) \cdot \check{c}_{\text {tot }}^{-1}\left(E, \nabla_{0}\right)=1+j\left(\widehat{c_{\text {tot }}}\left(\nabla_{0}, \nabla_{1}\right)\right)$.

Let now $K_{0}^{\bar{\partial}}(X)$ be the subgroup of $K_{0}^{\nabla}(X)$ generated by algebraic (resp. holomorphic) vector bundles endowed with compatible connexions. Zucker and Brylinski showed that $\check{c}_{\text {tot }}$ maps $K_{0}^{\bar{\partial}}(X)$ to a proper subgroup $\widehat{H R}^{2} \bullet(X, \mathbb{Z}(\bullet))$ of $\widehat{H}^{2 \bullet}(X, \mathbb{Z}(\bullet))$ (the subgroup of "restricted differential characters" the notation is Brylinski's one [11], the group is denoted $H_{\mathcal{G}}^{2 \bullet}(X, \mathbb{Z}(\bullet))$ by Zucker [39]). It follows from [11], 33 , and [39], (3.5.1) and (3.5.2), that $\widehat{H R}^{2 r}(X, \mathbb{Z}(r))$ is the $2 r$-th hypercohomology group of the complex of sheaves:

$$
0 \rightarrow \mathbb{Z} \stackrel{(2 \pi i)^{r}}{\longrightarrow} A_{L}^{<2 r}(X) \longrightarrow A_{\bar{L}}^{\geq 2 r}(X) / F_{r L} A_{\bar{L}}^{\geq 2 r}(X)
$$

where the differential forms are here taken logarithmic, $A_{L}^{2 r-1}(X)$ maps to $A_{L}^{2 r}(X) / F_{r L} A_{L}^{2 r}(X)$ and $F_{r L}$ stands as before for the Hodge filtration. For any $r$, let $\wedge_{0 L}^{2 r-1}(X)$ (resp. $\wedge_{F_{r L}}^{2 r-1}$ ) be the subspace of $\wedge_{L}^{2 r-1}(X)$ consisting of closed logarithmic forms whose cohomology class is integral (resp. of logarithmic forms whose coboundary lies in $\left.F_{r L} \wedge_{L}^{2 r}(X)\right)$, the right adaptation to $\widehat{H R}$ of the second exact sequence of [12], Thm. 1.1, then becomes the second line of the following commutative diagram:

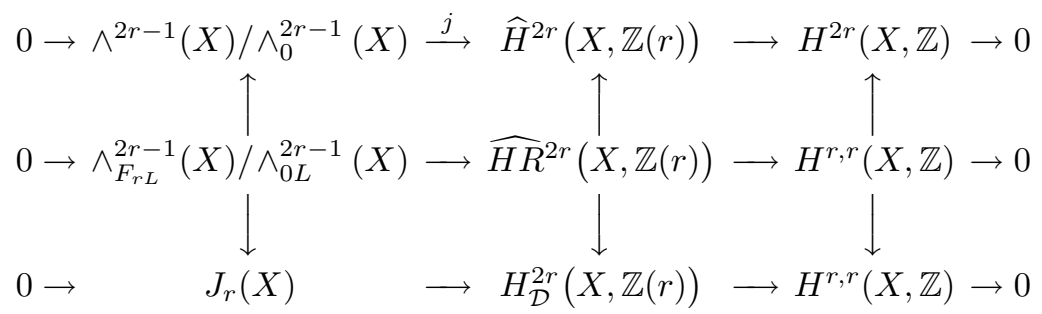

TOME $130-2002-\mathrm{N}^{\mathrm{O}} 2$ 


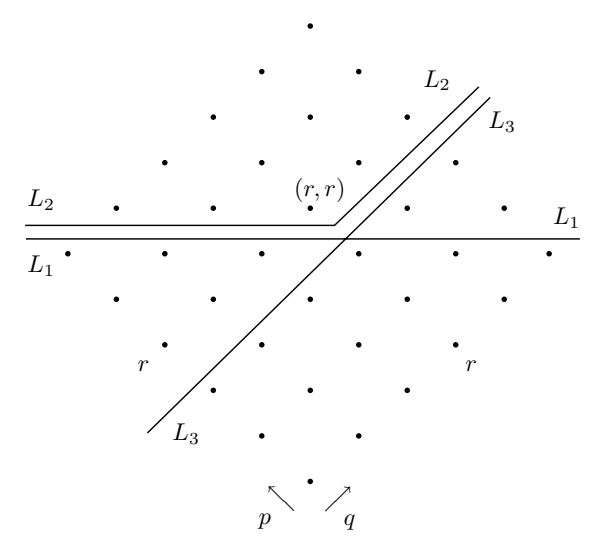

Figure 1. The Hodge diamond

which is obtained from the obvious maps from the complex of sheaves (26) to (23) and to the complex appearing in (22). The advantage of $\widehat{H R}^{2 \bullet}(X, \mathbb{Z}(\bullet))$ is that there is no reasonable map from Cheeger-Simons' differential characters to Hodge-Deligne cohomology (see [11], Lemma 2 and [39], (3.5.2) and (3.5.3)). In fact (for $X$ compact, to avoid logarithmic complications), the situation is illustrated as follows in the Hodge diamond (see Fig. 1): $\widehat{H}^{2 r}(X, \mathbb{Z}(r))$ (resp. $\widehat{H R}^{2 r}(X, \mathbb{Z}(r))$ resp. $\left.H_{\mathcal{D}}^{2 r}(X, \mathbb{Z}(r))\right)$ is obtained by sending $(2 \pi i)^{r} \mathbb{Z}$ into the de Rham complex quotiented by the subcomplex lying above $L_{1}$ (resp. $L_{2}$, resp. $\left.L_{3}\right)$, from which the maps $\widehat{H R}^{2 r}(X, \mathbb{Z}(r)) \rightarrow \widehat{H}^{2 r}(X, \mathbb{Z}(r))$ and $\widehat{H R}^{2 r}(X, \mathbb{Z}(r)) \rightarrow H_{\mathcal{D}}^{2 r}(X, \mathbb{Z}(r))$ are easily obtained.

Finally, Zucker [39], Thm. (5.2.1), and Brylinski [11], Thm. 4, show that the following diagram commutes for any $r$ :

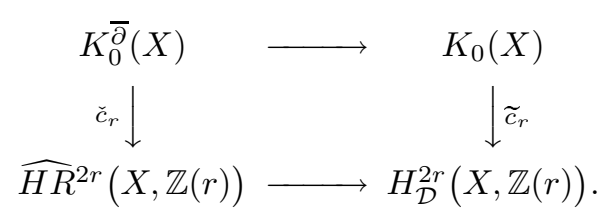

The theorem then follows from this, the left bottom square of the Diagram (27) above, Definition 3.4 and (25) which obviously holds with $\widehat{c_{\text {tot }} L}$ instead of $\widehat{c_{\text {tot }}}$. (Note that different compatible connexions will give different restricted differential characters but the same classes in Deligne-Beilinson cohomology, and that quotienting by $\wedge_{0}(X)$ instead of $\mathrm{d} \wedge(X)$ does not matter since $J_{r}(X)$ is obtained by quotienting by integral cohomology.)

BULletin DE LA SOCiÉtÉ MATHÉmATiQUe DE FRANCE 
REMARK. - Part $(\alpha)$ of Theorem 3.5 is a consequence of this result but it seemed more relevant to prove it as directly as possible.

REMARK. - A first version of the proof used Karoubi's "multiplicative" $K$ theory [30], [28] and [29]: for an additive invariant polynomial $P$, consider the free abelian group generated by triples $(E, \nabla, \alpha)$ where $E$ is a $\mathcal{C}^{\infty}$ vector bundle on $X, \nabla$ a connexion on $E$ and $\alpha \in \wedge_{(L)}^{2 \bullet-1}(X) / F_{\bullet}(L)$ is such that

$$
P\left(\nabla^{2}\right)-\mathrm{d} \alpha \in F_{\bullet(L)} \wedge_{(L)}^{2 \bullet}(X) .
$$

Karoubi [29], $\S \S 4$ and 5.1, defines the group $M K_{0}^{P}(X)$ as the quotient of this group by the relations

(A) $(E, \nabla, \alpha)+\left(F, \nabla^{\prime}, \beta\right)=\left(E \oplus F, \nabla \oplus \nabla^{\prime}, \alpha+\beta\right)$;

(B) if $\left(E, \nabla_{E}, \alpha\right)$ verifies (28), if $f: E \stackrel{\sim}{\rightarrow} F$ is a $\mathcal{C}^{\infty}$ isomorphism and $\nabla_{F}$ is any connexion on $F$ then $\left(E, \nabla_{E}, \alpha\right)=\left(F, \nabla_{F}, \alpha+\bar{P}\left(\nabla_{E}, f^{*} \nabla_{F}\right)\right)$.

One can adapt this to the multiplicative polynomial $c_{\text {tot }}$ by replacing (A) by

$$
(E, \nabla, \alpha)+\left(F, \nabla^{\prime}, \beta\right)=\left(E \oplus F, \nabla \oplus \nabla^{\prime}, \alpha \wedge c_{\text {tot }}\left(\nabla^{\prime 2}\right)+c_{\text {tot }}\left(\nabla^{2}\right) \wedge \beta\right),
$$

(B) and (28) staying unchanged. Using this theory one can obtain the following commutative diagram with exact lines:

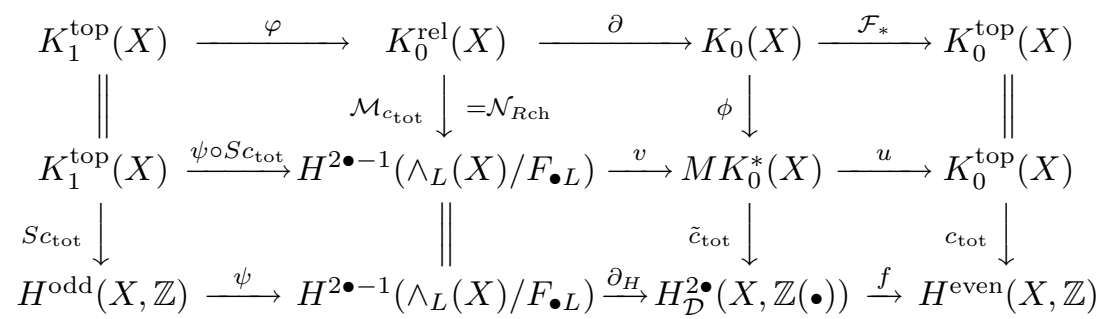

the third line is the second line of diagram (2) above. In the second line, $M K_{0}^{*}(X)$ stands for either $M K_{0}^{R c h}(X)$ ("additive" theory) or for $M K_{0}^{c_{\text {tot }}}(X)$ ("multiplicative" theory). The map $v$ is $\gamma \mapsto(E, \nabla, \alpha+\gamma)-(E, \nabla, \alpha)$ in the first case and $\gamma \mapsto\left(E, \nabla, \alpha+c_{\text {tot }}\left(\nabla^{2}\right) \wedge \gamma\right)-(E, \nabla, \alpha)$ in the second case (for any $(E, \nabla, \alpha)$ verifying (28) in both cases), the exactness of the second line is provided by a straightforward adaptation of [29], Thm. 5.3. Note that $\phi([E])=(E, \nabla, 0)$ for any compatible connexion $\nabla$ on $E$ and $u(E, \nabla, \alpha)=[E]$, the commutativity of the three "top" squares is elementary.

The map $\widetilde{c}_{\text {tot }}: M K_{0}^{R \operatorname{ch}}(X) \rightarrow H_{\mathcal{D}}^{2 \bullet}(X, \mathbb{Z}(\bullet))$ is then constructed as in [30], 7.19 (the target space is in [30] a "multiplicative cohomology" group which is shown to be isomorphic to $H_{\mathcal{D}}^{2 \bullet}(X, \mathbb{Z}(\bullet))$ in [30], Thm. 7.16). One step in this construction is to deduce by formal computations a relation of the form $c_{\text {tot }}\left(\nabla^{2}\right)-\mathrm{d} \beta \in F_{\bullet L} \wedge_{L}^{2 \bullet}(X)$ from the corresponding property for the Chern character $\operatorname{ch}\left(\nabla^{2}\right)-\mathrm{d} \alpha \in F_{\bullet L} \wedge_{L}^{2 \bullet}(X)$ (this is because Karoubi uses $\left.M K_{0}^{\mathrm{ch}}\right)$. This operation is of course not needed if one uses $M K_{0}^{c_{\text {tot }}}$ and very simply adapted

TOME $130-2002-\mathrm{N}^{\mathrm{O}} 2$ 
to the case of $M K_{0}^{R \mathrm{ch}}$. This latter case keeps the advantage that the diagram commutes with the equality between the two factors $H^{2 \bullet-1}\left(\wedge_{L}(X) / F_{\bullet L}\right)$ (there are multiplicative factors in the case of $M K_{0}^{\mathrm{ch}}$ due to the fact that $\psi \circ S c_{\text {tot }}$ must be replaced by $\psi \circ S \mathrm{ch}$ ). One then obtains as in, [30], 7.21-7.24 that the third column equals the total Chern class with values in Hodge Deligne cohomology. The central rectangle of this diagram ensures the result.

Finally the paper by Gillet and Soulé [21] also contains suitable information to prove the theorem, but it would be more complicated since their theory uses metrised vector bundles and real differential characters instead of complex ones.

\section{Applications}

Here I present some consequences of the preceding results for families of vector bundles. This section is organized as follows: in 5.1, the relevant subgroups of $K_{0}^{\mathrm{rel}}(X)$ and $K_{0}^{\mathrm{hom}}(X)$ for studying families of holomorphic (algebraic) vector bundles are presented. Some results on Nadel's classes restricted to these subgroups allow to give in 5.2 some monodromy restriction for families of holomorphic (algebraic) vector bundles which generalise [35], Thm. 5.1. Finally in 5.3, the case of holomorphic (algebraic) families is studied, yielding to a natural question about the possibility to describe the Albanese map of moduli spaces of vector bundles using Nadel's classes. All the results are stated in the holomorphic/complex analytic case the corresponding results in the algebraic case are deduced by replacing everywhere "holomorphic" by "algebraic", imposing the logarithmic condition to differential forms and adding the subscript $L$ where needed.

5.1. Deformation of vector bundles and $\boldsymbol{K}$-theory. - Let $\xi$ be a $\mathcal{C}^{\infty}$ complex vector bundle on $[0,1] \times X$ such that each restriction $\xi_{t}$ of $\xi$ to $\{t\} \times X$ is holomorphic on $X$ and let $\nabla$ be a connexion on $\xi$ such that its restriction to $\xi_{t}$ is compatible for any $t$. Let $f: \xi_{0} \rightarrow \xi_{1}$ be the isomorphism given by parallel transport by $\nabla$.

LEMmA. - The element $\left(\xi_{0}, \xi_{1}, f\right) \in K_{0}^{\mathrm{rel}}(X)$ is independent on the choice of the "compatible" connexion $\nabla$ on $\xi$.

Proof. - A continuous modification of $\nabla$ induces a continuous isotopy of $f$ which does not affect the class of $\left(\xi_{0}, \xi_{1}, f\right)$ in $K_{0}^{\text {rel }}(X)$, and the set of compatible connexions is convex. Moreover this proves that the element of $K_{1}^{\text {top }}(X)$ provided by the "difference" of the isomorphisms given by two complex analytic compatible connexions vanish. This implies the result in the algebraic case where the convexity (and the connexity) of the set of algebraic compatible connexions fails. 
DEFINITION 5.1. - Such elements of $K_{0}^{\mathrm{rel}}(X)$ are called deformations of bundles. The subgroup of $K_{0}^{\mathrm{rel}}(X)$ generated by them is denoted by $K_{0}^{\operatorname{def}}(X)$. The induced subgroup of $K_{0}^{\mathrm{hom}}(X)$ is denoted by $K_{0}^{\mathrm{cont}}(X)$.

(This would mean group of virtual vector bundles continuously equivalent to zero.)

Consider now a $\mathcal{C}^{\infty}$ connected manifold $Y$ and a $\mathcal{C}^{\infty}$ complex vector bundle $\xi$ on $Y \times X$ such that each restriction $\xi_{y}$ of $\xi$ to $\{y\} \times X$ is holomorphic on $X$. Let $\nabla$ be a connexion on $\xi$ whose restrictions to $\xi_{y}$ are compatible for all $y \in Y$.

DEFInition. - Such a data is called a family of holomorphic bundles on $X$. If $Y$ is complex analytic and $\xi$ is holomorphic on $Y \times X$, the family is called an holomorphic family.

REMARK. - Any family of algebraic bundles is the $\left(\mathcal{C}^{\infty}\right)$ pullback of some algebraic family; so there always exists some compatible connexion. (Thus $K_{0}^{\text {def }}(X)$ corresponds throw the Chern character to the subgroup $\mathrm{CH}_{\text {alg }}(X)$ of cycles on $X$ which are algebraically equivalent to 0 (up to torsion). Of course the notation $K_{0}^{\text {alg }}$ is not very satisfactory.)

Fix some basepoint $y_{0} \in Y$ and consider for any $y \in Y$ the element constructed as above $\left(\xi_{y_{0}}, \xi_{y}, f_{\gamma}\right) \in K_{0}^{\text {def }}(X)$ using any path $\gamma:[0,1] \rightarrow Y$ such that $\gamma(0)=y_{0}$ and $\gamma(1)=y$.

LEMMA. - This element depends on the path $\gamma$ only through its homotopy class, it does not depend on the choice of the "compatible" connexion $\nabla$.

Proof. - A continuous deformation of $\gamma($ or $\nabla$ ) induces a continuous isotopy of $f_{\gamma}$ which does not affect the class of $\left(\xi_{y_{0}}, \xi_{y}, f_{\gamma}\right)$ in $K_{0}^{\mathrm{rel}}(X)$ and hence in $K_{0}^{\text {def }}(X)$, the same argument as in the preceding lemma works in the algebraic case.

Corollary 5.2. - To a family of holomorphic vector bundles as above with fixed base point $y_{0}$ one can associate a map $Y \rightarrow K_{0}^{\operatorname{def}}(X)$ if $Y$ is simply connected. Otherwise, one gets a map $Y \rightarrow K_{0}^{\text {cont }}(X)$. In any case, one has maps $\pi_{i}(Y) \rightarrow K_{i}^{\mathrm{top}}(X)$ (where $\pi_{i}(Y)$ is the $i$-th homotopy group of $Y$ ).

Proof. - The lemma allows to construct a map $Y \rightarrow K_{0}^{\text {def }}(X)$ if $Y$ is simply connected because two paths joining $y_{0}$ and $y$ are always homotopic in $Y$. Otherwise, two elements $\left(\xi_{y_{0}}, \xi_{y}, f_{\gamma_{1}}\right)$ and $\left(\xi_{y_{0}}, \xi_{y}, f_{\gamma_{2}}\right)$ associated to two (non homotopic) paths $\gamma_{1}$ and $\gamma_{2}$ differ in $K_{0}^{\text {rel }}(X)$ by the element $\left(\xi_{y_{0}}, \xi_{y_{0}}, f_{\gamma_{2}}^{-1} \circ f_{\gamma_{1}}\right)$ which lies in $\varphi\left(K_{1}^{\mathrm{top}}(X)\right)$ (because of relation (vi) and Proposition 2.2) so that they define the same class in $K_{0}^{\text {def }}(X) / \varphi\left(K_{1}^{\text {top }}(X)\right)=K_{0}^{\text {cont }}(X)$. The last maps are classical (see Remark 5.7).

TOME $130-2002-\mathrm{N}^{\mathrm{O}} 2$ 
5.2. Rigidity and monodromy restrictions for families. - Denote by $F_{r} H^{k}\left(\wedge(X) / F_{s}\right)$ (for $\left.r \leq s\right)$, the subspace of $H^{k}\left(\wedge(X) / F_{s}\right)$ consisting of classes which can be represented by differential forms lying in $F_{r}$. Of course if $X$ is compact Kähler,

$$
F_{r} H^{k}\left(\wedge(X) / F_{s}\right)=\bigoplus_{\substack{p+q=k \\ r \leq p \leq s-1}} H^{p, q}(X)
$$

Lemma 5.3. - For any $(E, F, f) \in K_{0}^{\text {def }}(X)$ and any invariant additive (resp. multiplicative) polynomial $P$ (resp. $Q)$ :

$$
\mathcal{N}_{P}(E, F, f) \quad\left(\operatorname{resp} . \mathcal{M}_{Q}(E, F, f)\right) \in \bigoplus_{r=1}^{\operatorname{dim} X} F_{r-1} H^{2 r-1}\left(\wedge(X) / F_{r}\right)
$$

( $=\bigoplus_{p=1}^{\operatorname{dim} X} H^{p-1, p}(X)$ if $X$ is compact Kähler).

Proof. - Let $\left(\xi_{0}, \xi_{1}, f\right)$ be as before let $\nabla$ be a compatible connexion on $\xi$ and let $\nabla_{t}$ be the induced connexion on the restriction $\xi_{t}$ of $\xi$ to $\{t\} \times X$. For any $t$, $\nabla_{t}$ is compatible with the holomorphic structure of $\xi_{t}$ so that its curvature $\nabla_{t}^{2}$ is of complex type $(2,0)+(1,1)$. Now $\partial \nabla_{t} / \partial t$ is of type $(1,0)+(0,1)$ so that using formula (5), one sees that $\bar{P}\left(\nabla_{0}, \nabla_{1}\right)$ lies in $\bigoplus_{r} F_{r-1} \wedge^{2 r-1}(X)$. Its class in $H^{2 \bullet-1}\left(\wedge(X) / F_{\bullet}\right)$ then clearly lies in $F_{\bullet-1} H^{2 \bullet-1}\left(\wedge(X) / F_{\bullet}\right)$.

Corollary. - Consider a family of holomorphic vector bundles on $X$ over a pointed connected base $Y$.

If $Y$ is simply connected, the map of Corollary 5.2 composed with $\mathcal{N}_{P}$ (resp. $\mathcal{M}_{Q}$ ) for any invariant additive (resp. multiplicative) polynomial $P$ (resp. $Q$ ), gives rise to a continuous map

$$
Y \longrightarrow F_{\bullet-1} H^{2 \bullet-1}\left(\wedge(X) / F_{\bullet}\right)
$$

$\left(=\bigoplus_{p=1}^{\operatorname{dim} X} H^{p-1, p}(X)\right.$ if $X$ is compact Kähler $)$.

Otherwise, in view of Lemma 4.2, the map of Corollary 5.2 composed with $\mathcal{N}_{\text {Rch }}$ (resp. $\mathcal{M}_{c_{\text {tot }}}$ ) give rise to a continuous map

$$
Y \longrightarrow \bigoplus_{p=1}^{\operatorname{dim} X} \widetilde{J}_{p}(X)
$$

where $\widetilde{J}_{p}(X)$ is the subgroup $F_{p-1} H^{2 p-1}\left(\wedge(X) / F_{p}\right) / \psi\left(H^{2 p-1}(X, \mathbb{Z})\right)$ (equal to $H^{p-1, p}(X) / \psi\left(H^{2 p-1}(X, \mathbb{Z})\right)$ if $X$ is compact Kähler $)$ of $J_{p}(X)$.

REMARK. - In fact for projective $X$, the image of the map (30) lies in Liebermann's Jacobian (see [31], Def. 12.26) because of Theorem 1.1 (cf. Remark $5.9)$.

BUlletin DE LA SOCiÉtÉ MATHÉmATiQUe DE FRANCE 
COROLlary 5.4. - In particular, the range of the map $\pi_{1}(Y) \rightarrow K_{1}^{\mathrm{top}}(X)$ is included in $\left(\psi \circ S c_{\mathrm{tot}}\right)^{-1}\left(F_{\bullet-1} H^{2 \bullet-1}\left(\wedge(X) / F_{\bullet}\right)\right)$ (or equivalently there is a group morphism $\pi_{1}(Y) \rightarrow F_{\bullet-1} H^{2 \bullet-1}\left(\wedge(X) / F_{\bullet}\right)$ which factors through $\left.K_{1}^{\mathrm{top}}(X)\right)$.

REMARK 5.5. - This corollary provides the monodromy restriction which generalises Nadel's assertion [35], §10 (see also the example [35], §11).

Some extra restriction can be obtained in the two following situations:

Proposition 5.6. - If the Grothendieck K-theory class of $\xi_{y}$ is independent on $y \in Y$, then the two first maps of Corollary 5.2 vanish. In particular the map $\pi_{1}(Y) \rightarrow K_{1}^{\mathrm{top}}(X)$ takes its values in the torsion part of $K_{1}^{\mathrm{top}}(X)$.

If the compatible connexion $\nabla$ is such that its restrictions to the $\xi_{y}$ are all holomorphic connexions, then the two first maps of Corollary 5.2 take their values in $H^{1}\left(\wedge(X) / F_{1}\right)\left(=H^{0,1}(X)\right.$ if $X$ is Kähler $)$ or $\widetilde{J}_{1}(X)\left(=J_{1}(X)\right.$ in any case). In particular the Chern classes $\widetilde{c}_{r}\left(\xi_{y}\right) \in H_{\mathcal{D}}^{2 r}(X, \mathbb{Z}(r))$ are independent of $y$ for $r \geq 2$.

Holomorphic connexions are defined to have holomorphic connexion forms in holomorphic trivialisations of the vector bundle: this is equivalent to have a curvature form of complex type $(2,0)$. The second part of this proposition gives a new proof of the rigidity result by Esnault and Srinivas (see [16], Prop. 1) (this result of course also applies for flat bundles for which it is well known).

Proof. - If for any $y,\left[\xi_{y}\right]=\left[\xi_{y_{0}}\right] \in K_{0}(X)$, then because of exact sequence (1), $\left(\xi_{y_{0}}, \xi_{y}, f_{\gamma}\right)$ lies in $\varphi\left(K_{1}^{\mathrm{top}}(X)\right)$ which is a discrete subgroup of $K_{0}^{\mathrm{rel}}(X)$ so that the map $Y \rightarrow K_{0}^{\text {cont }}(X)$ vanishes. The map $Y \rightarrow K_{0}^{\operatorname{def}}(X)$ (if $Y$ is simply connected) is locally constant, and it vanishes at the point $\xi_{y_{0}}$. This proves the first assertion (provided $Y$ is connected). Because of Lemma 4.2, this yields that the elements of $K_{1}^{\text {top }}(X)$ lying in the image of $\pi_{1}(Y)$ have vanishing total Chern class in $H^{2 \bullet-1}\left(\wedge(X) / F_{\bullet}\right)$. The map $H^{\text {odd }}(X, \mathbb{Q}) \rightarrow H^{2 \bullet-1}\left(\wedge(X) / F_{\bullet}\right)$ is injective: this ensures that the total Chern class of those elements in fact vanish in $H^{\text {odd }}(X, \mathbb{Q})$. The second assertion follows now from the Chern isomorphism $K_{1}^{\text {top }}(X) \otimes \mathbb{Q} \cong H^{\text {odd }}(X, \mathbb{Q})$.

In the case of holomorphic connexions, the first assertion follows from a direct adaptation of the proof of Lemma 5.3, the second assertion is now a direct corollary of Theorem 4.6

REMARK 5.7. - An immediate generalisation of Lemma 5.3 yields the following "higher" monodromy restriction:

Proposition 5.8. - An element $\alpha$ of $K_{i}^{\mathrm{top}}(X)$ cannot be realised as a deformation of holomorphic bundles over $S^{i} \times X$ (where $S^{i}$ is the $i$-th dimensional real sphere) unless its $i$-th suspended Chern class in complex cohomology

TOME $130-2002-\mathrm{N}^{\mathrm{O}} 2$ 
$S^{i} c_{\text {tot }}(\alpha)$ lies in $\oplus_{r} F_{r-i} H^{2 r-i}(X, \mathbb{C}) \quad\left(\right.$ or $\bigoplus_{|p-q| \leq i} H^{p, q}(X)$ if $X$ is Kähler $)$ (or equivalently if one has a deformation of holomorphic bundles on $Y \times X$, one obtains group morphisms $\pi_{i}(Y) \rightarrow F_{\bullet-i} H^{2 \bullet-i}(X, \mathbb{C})$ which factor throw $\left.K_{i}^{\mathrm{top}}(X)\right)$.

Proof. - As for $i=1$ one has

$$
K_{i}^{\mathrm{top}}(X) \cong \operatorname{Ker}\left(K_{0}^{\mathrm{top}}\left(S^{i} \times X\right) \rightarrow K_{0}^{\mathrm{top}}(\{\mathrm{pt}\} \times X)\right)
$$

(the map corresponding to the restriction to some point $\{\mathrm{pt}\} \subset S^{i}$ ), so that any vector bundle on $S^{i} \times X$ defines some class in $K_{i}^{\mathrm{top}}(X)$. The suspended total Chern class is defined in a similar way as in the case of $K_{1}^{\text {top }}$, its ChernWeil version is obtained by integrating over $S^{i}$ the Chern-Weil form on $S^{i} \times X$ associated to the total Chern class of $E$ calculated with any connexion on $E$ and wedging it with the inverse of the total Chern class of $\left.E\right|_{\{\mathrm{pt}\} \times X}$ (this last operation is needed because $c_{\text {tot }}$ is multiplicative (as in (15)), it is not neded for additive classes). If $E$ (on $S^{i} \times X$ ) is a deformation of holomorphic bundles and if $\nabla$ is compatible, the suitable analogue of formula (5) yields that the calculated differential form has types $(p, q)$ with $q \leq p+i$. The condition $|p-q| \leq i$ results from the fact that the calculated classes are integral (hence real).

Now if $X$ is algebraic, such a family is the $\mathcal{C}^{\infty}$ pullback of some algebraic family on $Z \times X$ for some algebraic variety $Z$. If $i=2$ and $S^{2}$ is homologous to some rational curve in $Z$, one will obtain that $S^{2} c_{\text {tot }}$ will lie in $\bigoplus_{p} H^{p, p}(X)$ instead of $\bigoplus_{|p-q| \leq 2} H^{p, q}(X)$ because all restrictions to $\{\mathrm{pt}\} \times X$ of an algebraic vector bundle on $\mathbb{P}^{1} \times X$ lie in the same Grothendieck $K$-theory class. This is a generalisation to $K_{2}^{\text {top }}$ of the first case of extra restriction above.

\subsection{The case of holomorphic families}

Proposition. - If $Y$ is a complex analytic manifold and $\xi$ is a holomorphic vector bundle over $Y \times X$, then the maps $Y \rightarrow F_{\bullet-1} H^{2 \bullet-1}\left(\wedge(X) / F_{\bullet}\right)$ if $Y$ is simply connected, or $Y \rightarrow \widetilde{J}_{\bullet}(X)$ otherwise, are holomorphic.

Proof. - Formula (5) for transgression yields for a choice of path $\gamma$ from $y_{0}$ to $y$ :

$$
\bar{P}\left(\nabla^{\xi_{y_{0}}}, f_{\gamma}^{*} \nabla^{\xi_{y}}\right)=\int_{\gamma} P\left(\nabla^{2}\right)
$$

where $\nabla$ is any connexion on $\xi$ over $Y \times X$. Then on $Y$

$$
\mathrm{d}_{Y} \bar{P}\left(\nabla^{\xi_{y_{0}}}, f_{\gamma}^{*} \nabla^{\xi_{y}}\right)=\left[P\left(\nabla^{2}\right)\right]^{(1)}
$$

where $\mathrm{d}_{Y}$ is the exterior differential along $Y$ and $\left[P\left(\nabla^{2}\right)\right]^{(1)}$ stands for the degree one part of $P\left(\nabla^{2}\right)$ on $Y$ (which is a one form on $Y$ with values in 
$\left.\wedge^{\text {odd }}(X)\right)$. Choose now $\nabla$ to be compatible with the holomorphic structure of $\xi$ over $Y \times X$. Then $P\left(\nabla^{2}\right)$ lies in $\bigoplus_{r=0}^{\operatorname{dim} X} F_{r} \wedge^{2 r}(Y \times X)$ so that

$$
\begin{aligned}
& {\left[P\left(\nabla^{2}\right)\right]^{(1)} \in \wedge^{1,0}(Y) \otimes\left(\bigoplus_{r=1}^{\operatorname{dim} X} F_{r-1} \wedge^{2 r-1}(X)\right) } \\
& \bigoplus \wedge^{0,1}(Y) \otimes\left(\bigoplus_{r=1}^{\operatorname{dim} X} F_{r} \wedge^{2 r-1}(X)\right) .
\end{aligned}
$$

Now $\mathcal{N}_{P}\left(\xi_{y_{0}}, \xi_{y}, f_{\gamma}\right)$ is the class of $\bar{P}\left(\nabla^{\xi_{y_{0}}}, f_{\gamma}^{*} \nabla^{\xi_{y}}\right)$ modulo $\bigoplus_{r=1}^{\operatorname{dim} X} F_{r} \wedge^{2 r-1}(X)$ so that the second part of the right hand side in (31) does not contribute to $\mathrm{d}_{Y} \mathcal{N}_{P}\left(\xi_{y_{0}}, \xi_{y}, f_{\gamma}\right)$. So $\mathrm{d}_{Y} \mathcal{N}_{P}\left(\xi_{y_{0}}, \xi_{y}, f_{\gamma}\right) \in \wedge^{1,0}(Y) \otimes H^{2 \bullet-1}\left(\wedge(X) / F_{\bullet}\right)$ this proves the result. That the same holds for $\mathcal{M}_{P}$ is straightforward (in the algebraic case, this argument proves the holomorphicity of the maps, not their algebraicity).

REMARK 5.9. - This last result should not be surprising, at least for projective $X$, in view of Theorem 1.1 and the fact that the Abel-Jacobi map is analytic (see [31], Def. (12.23) and Cor. (12.28)). Other classical results for Abel-Jacobi maps apply here if $X$ is projective, in particular $\mathcal{M}_{c_{\text {tot }}}$ is a universal map from $K_{0}^{\text {cont }}(X)$ to Abelian varieties if $\operatorname{dim}(X) \leq 3$ (see [34]) up to 2-torsion (see Remark 4.4).

Consider some flat line bundle $L$ on $X$ and its class $[L]$ in $\operatorname{Pic}^{0}(X) \cong J_{1}(X)$ and take some global nonvanishing $\mathcal{C}^{\infty}$ section $f$ of $L$. It can be interpreted as a $\mathcal{C}^{\infty}$ isomorphism $f: \mathbb{C} \rightarrow L$ from the trivial line bundle to $L$.

\section{Proposition 5.10. - Modulo integral cohomology}

$$
\mathcal{N}_{c_{1}}(\mathbb{C}, L, f)=-[L] \in J_{1}(X) \cong \operatorname{Pic}^{0}(X) .
$$

Proof. - Take some open cover of $X$ where $L$ trivialises. Let $A^{0,0 *}$ be the sheaf on $X$ of $\mathcal{C}^{\infty}$ nonvanishing functions. In the trivialisation of $L, f$ defines a cochain in $C^{0}\left(X, A^{0,0 *}\right)$ whose Čech coboundary $\delta f$ gives the cocycle defining $L$ when seen as an element of $H^{1}\left(X, \mathcal{O}_{X}^{*}\right)$. Its image in $H^{2}(X, \mathbb{Z})$ in the long exact sequence in cohomology associated to

$$
0 \rightarrow \mathbb{Z} \longrightarrow \mathcal{O}_{X} \stackrel{\mathrm{e}^{2 \pi i}}{\longrightarrow} \mathcal{O}_{X}^{*} \rightarrow 0
$$

vanishes. $[L] \in \operatorname{Pic}^{0}(X)=H^{1}\left(X, \mathcal{O}_{X}\right) / H^{1}(X, \mathbb{Z})$ is then by definition the reduction modulo integral cohomology of the class of $(1 / 2 \pi i) \delta \log f$ (whose image by $\mathrm{e}^{2 \pi i}$ is $\left.\delta f\right)$.

From (5) (or [35], Def. p. 40 and Lemma 4.3)

$$
\mathcal{N}_{c_{1}}(\mathbb{C}, L, f)=\frac{i}{2 \pi} f^{-1} \bar{\partial} f=\frac{-1}{2 \pi i} \bar{\partial}(\log f)
$$

TOME $130-2002-\mathrm{N}^{\mathrm{O}} 2$ 
and its class in $H^{0,1}(X)$ corresponds to the class of $(-1 / 2 \pi i) \delta(\log f)$ throw the Dolbeault isomorphism $H^{0,1}(X) \cong H^{1}\left(X, \mathcal{O}_{X}\right)$ (which comes from the long exact sequence in cohomology associated to:

$$
0 \rightarrow \mathcal{O}_{X} \longrightarrow A^{0,0} \longrightarrow \mathcal{Z}_{\bar{\partial}}^{0,1} \rightarrow 0
$$

where $A^{0,0}$ and $\mathcal{Z}_{\bar{\partial}}^{0,1}$ are the sheaves on $X$ of $\mathcal{C}^{\infty}$ functions and of $\bar{\partial}$-closed $(0,1)$-type differential forms respectively).

Lemma 5.11. - For $(E, F, f) \in K_{0}^{\mathrm{rel}}(X)$, one has

$$
\mathcal{N}_{c_{1}}(E, F, f)=\mathcal{N}_{c_{1}}(\operatorname{det} E, \operatorname{det} F, \operatorname{det} f) .
$$

Proof. — This is a straightforward consequence of Nadel's results in [35], §9, and Lemma 4.3.

An application to moduli space theory could be the following: consider some moduli space $\widetilde{\mathcal{M}}$ of stable vector bundles on some algebraic manifold $X$. Imposing the restriction that the determinant bundle be fixed is in fact nothing but considering the kernel of the map

$$
\mathcal{N}_{c_{1}}: \widetilde{\mathcal{M}} \longrightarrow J_{1}(X) \quad\left(=\widetilde{J}_{1}(X)\right)
$$

$\mathcal{M}_{c_{\text {tot }}}$ provides then analytic maps from $\widetilde{\mathcal{M}}$ to all intermediate jacobians of $X$ and more precisely because of Lemma 5.3 into the $(\bullet, \bullet+1)$-complex type part of them, and their range are included in some abelian subvariety because of Theorem 1.1 and [31], Prop. 12.23.

One can raise the question in which cases does the factorisation of the map $\mathcal{M}_{c_{\text {tot }}}$ throw the Albanese variety of the considered moduli space yield an embedding of this Albanese variety into the intermediate jacobians of $X$ (or only a map with finite kernel: see Remark 4.4). This question is of course closely related to the conjecture that the Abel-Jacobi map is universal for the continuous part of the Chow group (see [31], 12.27, and Remark 5.9 above)

This is for example the case for most moduli spaces of vector bundles on an abelian surface: Yoshioka [38], [37] has exhibited the corresponding Albanese maps and it is shown in Subsection 6.5 below that Yoshioka's maps equal $\mathcal{M}_{c_{\text {tot }}}$.

Another example in dimension 3 is provided by Iliev, Markushevich and Tikhomirov [32], [24]: they have proved that the map $\mathcal{M}_{c_{2}}$ (or rather $\mathcal{N}_{R \mathrm{ch}_{2}}$ ) from some (Zariski) open subset of some moduli space $\mathcal{M}$ of rank two vector bundles on the cubic threefold $X$ to its intermediate jacobian $J_{2}(X)$ is etale $\left(J_{2}(X)\right.$ is neither the Jacobi nor the Albanese variety of $X$ : this makes the special interest of this example). That their map coïncides with $\mathcal{N}_{R \mathrm{ch}_{2}}$ can be proved as follows: they in fact construct

$$
\begin{aligned}
\mathcal{M} & \longrightarrow \mathcal{M}^{\prime} \stackrel{c_{2}}{\longrightarrow} \mathrm{CH}_{2} \stackrel{A J}{\longrightarrow} J_{2}(X), \\
\mathcal{E} & \longmapsto \mathcal{E}(1) \longmapsto[C]-\left[C_{0}\right] \longmapsto A J\left([C]-\left[C_{0}\right]\right),
\end{aligned}
$$

BULLETIN DE LA SOCIÉtÉ MATHÉMATIQUE DE FRANCE 
where $\mathcal{M}^{\prime}$ is the relevant associated moduli space, and $\mathcal{E}(1)$ is obtained from $C$ by Serre's construction. $\left[C_{0}\right] \in \mathrm{CH}_{2}(X)$ is associated to the the fixed element $\left[\mathcal{E}_{0}\right] \in \mathcal{M}$. Thus $c_{2}(\mathcal{E}(1))=[C]$ and in $\mathrm{CH}_{2}(X)$, one has

$$
c_{2}\left([\mathcal{E}]-\left[\mathcal{E}_{0}\right]\right)=c_{2}\left([\mathcal{E}(1)]-\left[\mathcal{E}_{0}(1)\right]\right)
$$

because $[\mathcal{E}]$ and $\left[\mathcal{E}_{0}\right]$ have same $c_{1}$ in $\mathrm{CH}_{1}(X)$. Thus using Theorem 1.1:

$$
A J\left(c_{2}\left([\mathcal{E}]-\left[\mathcal{E}_{0}\right]\right)\right)=\mathcal{N}_{R \mathrm{ch}_{2}}\left([\mathcal{E}]-\left[\mathcal{E}_{0}\right]\right) .
$$

\section{Direct image for relative $K$-theory}

This section is organised as follows: in 6.1, a basic resolution lemma for relative $K$-theory is proved, which allows to construct in 6.2 a direct image map from $K_{0}^{\mathrm{rel}}(X)$ to $K_{0}^{\mathrm{rel}}(Y)$ in the case of a proper submersion $\pi: X \rightarrow Y$ between quasiprojective manifolds, using a trick by Atiyah and Singer (see [2], Prop. 2.2, see also [3], §9.5). A Riemann-Roch theorem for Nadel's classes is then stated in 6.3 in the case where $X$ and $Y$ are compact, whose proof is reduced to the existence of some transgression form explicitely constructed in 6.4. The construction relies on Bismut and Köhler's double transgression theory for proper submersions [9] and uses the family index theorem for the $\bar{\partial}$ operator on non holomorphic vector bundles. This theorem is then used in 6.5 to prove that Yoshioka's Albanese maps from moduli spaces of vector bundles on abelian surfaces coïncide with $\mathcal{M}_{c_{\text {tot }}}$. Then in 6.6 , the direct image map from $K_{0}^{\text {def }}(Y)$ to $K_{0}^{\text {def }}(X)$ is constructed in the case of a closed immersion $\iota: Y \rightarrow X$ : this allows to prove in 6.7 the Riemann-Roch theorem in this case, using Bismut Gillet and Soulé's explicit double transgression of the Chern character [8].

6.1. Resolution to the right in relative $\boldsymbol{K}$-theory:- In the whole section, a vector bundle $\xi$ on $X$ will be called "acyclic" if the cohomology groups $H^{i}\left(X, \mathcal{O}_{X}(\xi)\right)$ vanish for any $i \geq 1$.

Lemma 6.1. - Consider a quasiprojective manifold $X$ and $(E, F, f) \in K_{0}^{\mathrm{rel}}(X)$. Then there exists two acyclic vector bundles $A_{E}$ and $A_{F}$ on $X$ and $a \mathcal{C}^{\infty}$ isomorphism $g: A_{E} \rightarrow A_{F}$ such that the following diagram

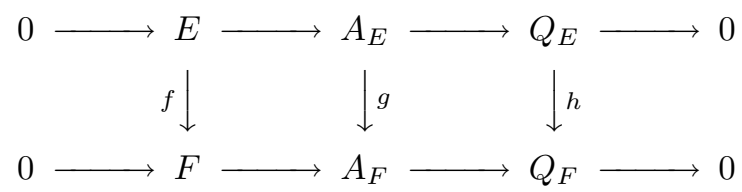

commutes and its lines are algebraic short exact sequences.

If $\left(f_{t}\right)_{t \in[0,1]}$ is an isotopy of $\mathcal{C}^{\infty}$ isomorphisms from $E$ to $F$, then there exists an isotopy $\left(g_{t}\right)_{t \in[0,1]}$ (resp. $\left.\left(h_{t}\right)_{t \in[0,1]}\right)$ of $\mathcal{C}^{\infty}$ isomorphisms from $A_{E}$ to $A_{F}$ (resp. from $Q_{E}$ to $Q_{F}$ ) such that the preceding diagram commutes for any $t$.

TOME $130-2002-\mathrm{N}^{\mathrm{O}} 2$ 
For any short algebraic exact sequence

$$
0 \rightarrow E^{\prime} \longrightarrow E \longrightarrow E^{\prime \prime} \rightarrow 0
$$

there is a commutative (algebraic) diagram

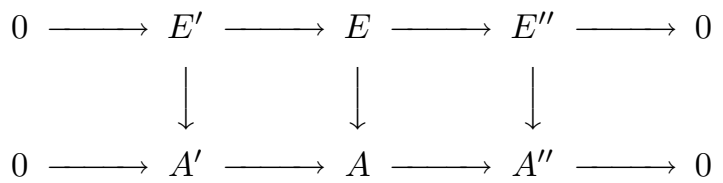

with both lines exact, $A^{\prime} A$ and $A^{\prime \prime}$ acyclic and injective "vertical" maps.

Proof. - In this case, $X$ has an ample line bundle $L$. There exists $n$ such that $L^{\otimes n}$ is generated by its global sections, so that one obtains an epimorphism $\mathbb{C}^{N} \rightarrow L^{\otimes n}$. Dualizing and tensoring with $L^{\otimes n}$ gives the exact sequence of vector bundles:

$$
0 \rightarrow \mathcal{O}_{X} \longrightarrow\left(L^{\otimes n}\right) \otimes \mathbb{C}^{N} \longrightarrow Q \rightarrow 0 .
$$

Integer $n$ can be chosen such that $E \otimes L^{\otimes n}$ and $F \otimes L^{\otimes n}$ are acyclic, the result is then given by tensoring this exact sequence by $E$ and $F$. The same argument obviously holds for $E^{\prime}, E$ and $E^{\prime \prime}$ as in the lemma.

LEMMA 6.2. - If one has two bundle monomorphisms $E \stackrel{i^{\prime}}{\rightarrow} A^{\prime}$ and $E \stackrel{i^{\prime \prime}}{\rightarrow} A^{\prime \prime}$, then there exists a third one $E \stackrel{i}{\rightarrow} A$ such that the diagram

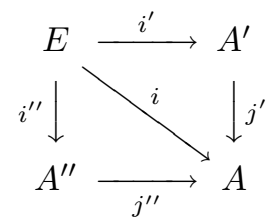

commutes, $j^{\prime}$ and $j^{\prime \prime}$ are monomorphisms and in $A$ :

$$
j^{\prime}\left(A^{\prime}\right) \cap j^{\prime \prime}\left(A^{\prime \prime}\right)=i(E) .
$$

Note also that the composed map $A^{\prime} / i^{\prime}(E) \hookrightarrow A / i(E) \rightarrow A / j^{\prime \prime}\left(A^{\prime \prime}\right)$ is an embedding (and the same holds for $\left.A^{\prime \prime} / i^{\prime \prime}(E) \hookrightarrow A / i(E) \rightarrow A / j^{\prime}\left(A^{\prime}\right)\right)$.

Proof. — Consider the monomorphism

$$
E \stackrel{i^{\prime} \oplus\left(-i^{\prime \prime}\right)}{\longrightarrow} A^{\prime} \oplus A^{\prime \prime}
$$

and take $A$ to be the quotient of $A^{\prime} \oplus A^{\prime \prime}$ by the image of $E$. Then the morphism $E \stackrel{i}{\rightarrow} A$ is the composed map

$$
E \stackrel{i^{\prime} \text { or } i^{\prime \prime}}{\longrightarrow} A^{\prime} \oplus A^{\prime \prime} \longrightarrow A
$$

(indifferently) and the stated properties are elementary.

BULLETIN DE LA SOCIÉTÉ MATHÉMATIQUE DE FRANCE 
Corollary 6.3. - There exist resolutions to the right by $\mathcal{C}^{\infty}$-isomorphic acyclic bundles in $K_{0}^{\mathrm{rel}}(X)$. For two resolutions

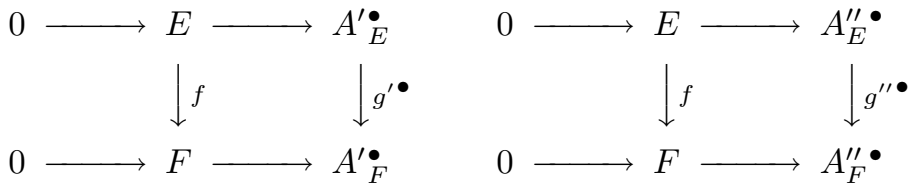

there exists a third one

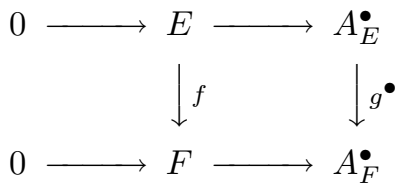

such that this diagram commutes

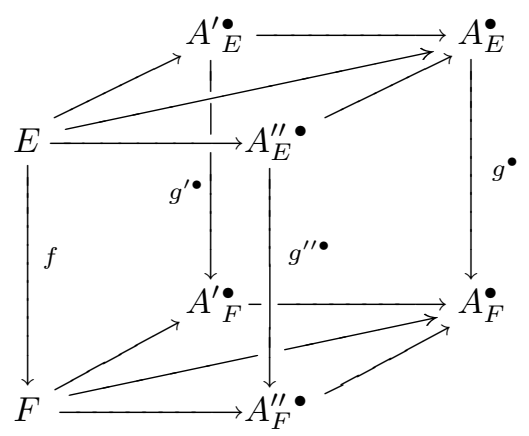

and all the "horizontal" maps are (graded) algebraic monomorphisms.

Proof. - It suffices to apply Lemma 6.1 to $(E, F, f)$ and then to $\left(Q_{E}, Q_{F}, h\right)$ and so on. The long exact sequence in cohomology associated to the first line of $(32)$ shows that if $H^{i}(X, E)$ vanishes for all $i \geq p>1$ then $H^{i}\left(X, Q_{E}\right)$ vanishes for $i \geq p-1$. Thus after $\operatorname{dim} X$ steps, the last quotients have vanishing cohomology groups in positive degrees.

Note that the relations defining $K_{0}^{\text {rel }}$ are compatible with such resolutions: this is obvious for (i) and (iii) and comes from the second and third parts of Lemma 6.1 for (ii) and (iv).

For constructing the "dominating" resolution, first apply Lemma 6.2 to the monomorphisms $E \rightarrow A^{\prime 0}$ and $E \rightarrow A_{E}^{\prime \prime 0}$ (resp. $F \rightarrow A_{F}^{\prime 0}$ and $F \rightarrow A_{F}^{\prime \prime 0}$ ) to obtain monomorphisms $E \rightarrow B_{E}^{0}$ and $F \rightarrow B_{F}^{0}$. One also obtains a $\mathcal{C}^{\infty}$ isomorphism $h^{0}: B_{E}^{0} \rightarrow B_{F}^{0}$ which is compatible with $f, g^{\prime 0}$ and $g^{\prime \prime 0}$. $B_{E}^{0}$ and $B_{F}^{0}$ need not be acyclic, apply Lemma 6.1 to $\left(B_{E}^{0}, B_{F}^{0}, h^{0}\right)$ to obtain $\left(A_{E}^{0}, A_{F}^{0}, g^{0}\right)$ which has the expected properties. The quotient bundles $Q_{E}^{\prime 0}=A_{E}^{\prime 0} / \operatorname{image}(E)$ and $Q_{E}^{\prime \prime 0}=A_{E}^{\prime \prime 0} /$ image $(E)$ of course imbed in $Q_{E}^{0}=A_{E}^{0} /$ image $(E)$, the same 
holds for ${Q_{F}^{\prime 0}}_{F}, Q_{F}^{\prime \prime 0}$ and $Q_{F}^{0}$ (with obvious notations) and these embeddings commute with the $\mathcal{C}^{\infty}$ isomorphisms ${Q^{\prime}}_{E} \stackrel{\sim}{\rightarrow} Q_{F}^{\prime 0}, Q_{E}^{\prime \prime 0} \stackrel{\sim}{\rightarrow} Q_{F}^{\prime \prime 0}$ and $Q_{E}^{0} \stackrel{\sim}{\rightarrow} Q_{F}^{0}$.

Suppose now that the dominating resolution has been constructed up to $\left(A_{E}^{k}, A_{F}^{k}, g^{k}\right)$ with imbeddings of the vector bundles $Q_{E}^{\prime k}=A_{E}^{\prime k} /$ image $\left(A_{E}^{\prime k-1}\right)$ and $Q_{E}^{\prime \prime k}=A_{E}^{\prime \prime k} /$ image $\left(A_{E}^{\prime \prime k-1}\right)$ into $Q_{E}^{k}=A_{E}^{k} /$ image $\left(A_{E}^{k-1}\right)$, the same holding for $F$ and all being compatible with the $\mathcal{C}^{\infty}$ isomorphisms. The technique is here to apply successively Lemma 6.2 to the monomorphisms $Q_{E}^{\prime k} \rightarrow Q_{E}^{k}$ and $Q_{E}^{\prime k} \rightarrow A_{E}^{\prime k+1}$ to obtain $Q_{E}^{\prime k} \rightarrow B_{E}^{k+1}$, then to $Q_{E}^{\prime \prime k} \rightarrow B_{E}^{k+1}$ (throw $Q_{E}^{k}$ ) and $Q_{E}^{\prime \prime k} \rightarrow A_{E}^{\prime \prime k+1}$ to obtain $Q_{E}^{\prime \prime k} \rightarrow C_{E}^{k+1}$. The same can be performed for $F$ and one obtains $C_{F}^{k+1}$ with some $\mathcal{C}^{\infty}$ isomorphism $h^{k+1}: C_{E}^{k+1} \rightarrow C_{F}^{k+1}$; finally apply Lemma 6.1 to $\left(C_{E}^{k+1}, C_{F}^{k+1}, h^{k+1}\right)$ to obtain $\left(A_{E}^{k+1}, A_{F}^{k+1}, g^{k+1}\right)$. The quotient vector bundle ${Q^{\prime k}}_{E}^{k+1}=A_{E}^{\prime k+1} / \operatorname{image}\left(A_{E}^{\prime k}\right)$ imbeds into $B_{E}^{k+1} / \operatorname{image}\left(Q_{E}^{k}\right)$ because of the last property stated in lemma 6.2 and then into $C_{E}^{k+1} / \operatorname{image}\left(Q_{E}^{k}\right)$ and in $A_{E}^{k+1} / \operatorname{image}\left(Q_{E}^{k}\right)$. In the same way, $Q_{E}^{\prime \prime k+1}$ imbeds trivially in $C_{E}^{k+1} /$ image $\left(Q_{E}^{\prime \prime k}\right)$ and also in $C_{E}^{k+1} /$ image $\left(B_{E}^{k+1}\right)$ because of the last property in Lemma 6.2: it thus imbeds into $C_{E}^{k+1} / \operatorname{image}\left(Q_{E}^{k}\right)$ too, because this last space is an intermediary quotient between the two formers. Consequently it imbeds then into $A_{E}^{k+1} / \operatorname{image}\left(Q_{E}^{k}\right)$ as desired. The same holds for $Q_{F}^{\prime k+1}$ and $Q_{F}^{\prime \prime k+1}$ and all these monomorphisms can be constructed to be compatible with the $\mathcal{C}^{\infty}$ isomorphisms. This finishes the induction.

6.2. The direct image morphism for proper submersions. - Consider now a surjective proper morphism $\pi: X \rightarrow Y$ between quasiprojective manifolds with regular fibres of constant dimension and consider some element $\left(A_{0}, A_{1}, g\right) \in K_{0}^{\text {rel }}(X)$ with $A_{0}$ and $A_{1}$ acyclic. I shall now construct the direct image of such an element of $K_{0}^{\mathrm{rel}}(X)$ in $K_{0}^{\mathrm{rel}}(Y)$, using the trick in [3], $\S 9.5$ (see [2], Prop. 2.2): consider two compatible connexions $\nabla_{0}$ and $\nabla_{2}$ on $A_{0}$ and $A_{1}$ and choose a homotopy $\left(\nabla_{t}\right)_{t \in[0,1]}$ between $\nabla_{0}$ and $\nabla_{1}=g^{*} \nabla_{2}$ on $A_{0}$, such that $\nabla_{t}$ is constant on $[0, \varepsilon)$ and $(1-\varepsilon, 1]$ for some $\varepsilon>0$; all these connexions can be chosen to be compatible with some fixed hermitian metric $h_{0}$ on $A_{0}$. Put some Kähler metric $\omega$ on $X$. Associated to these data, there is a family $\mathcal{D}_{t, y}$ of $\operatorname{Spin}^{c}$ Dirac operators acting on $\mathcal{C}^{\infty}\left(\pi^{-1}(y), \wedge^{0, \bullet}\left(\pi^{-1}(y)\right) \otimes A_{0}\right)$. One has for any $t$ and $y$ :

$$
\mathcal{D}_{t, y}^{+}: \mathcal{E}_{y}^{+} \longrightarrow \mathcal{E}_{y}^{-} \quad \text { and } \quad \mathcal{D}_{t, y}^{-}: \mathcal{E}_{y}^{-} \longrightarrow \mathcal{E}_{y}^{+}
$$

where $\mathcal{E}_{y}^{ \pm}:=\mathcal{C}^{\infty}\left(\pi^{-1}(y), \wedge^{0, \text { ovend }}\left(\pi^{-1}(y)\right) \otimes A_{0}\right)$. Consider some $\mathcal{C}^{\infty}$ bundle morphism $\psi: \mathbb{C}^{N} \rightarrow \mathcal{E}^{-}$defined on $[0,1] \times Y$ and set

$$
\begin{aligned}
\psi^{*}: \mathcal{E}^{-} & \longrightarrow \mathbb{C}^{N}, \\
\phi & \longmapsto \\
& \left(\left(\phi / \psi\left(e_{1}\right)\right),\left(\phi / \psi\left(e_{2}\right)\right), \ldots,\left(\phi / \psi\left(e_{N}\right)\right)\right),
\end{aligned}
$$

BULLETIN DE LA SOCiÉTÉ MATHÉMATIQUe DE FRANCE 
where $\left(e_{1}, e_{2}, \ldots, e_{N}\right)$ is the canonical base of $\mathbb{C}^{N}$, and $(/)$ is the $\mathrm{L}^{2}$ scalar product on $\mathcal{E}_{y}^{-}$associated to $h_{0}$ and $\omega$. Define then the modified Dirac operators

$$
\begin{aligned}
& \mathcal{D}_{t, y, \psi}^{+}: \mathcal{E}_{y}^{+} \oplus \mathbb{C}^{N} \stackrel{\mathcal{D}_{t, y}^{+}+\psi}{\longrightarrow} \mathcal{E}_{y}^{-}, \\
& \mathcal{D}_{t, y, \psi}^{-}: \mathcal{E}_{y}^{-} \stackrel{\mathcal{D}_{t, y}^{-} \oplus \psi^{*}}{\longrightarrow} \mathcal{E}_{y}^{+} \oplus \mathbb{C}^{N} .
\end{aligned}
$$

Then the same argument as in [3], Lemma 9.30 shows that for any compact subset $K \subset Y$, there are suitable choices of $N$ and $\psi$ such that $\mathcal{D}_{t, y, \psi}^{+}$is surjective on $\mathcal{E}_{y}^{-}$for any $(t, y) \in[0,1] \times K$. Then $\operatorname{Ker} \mathcal{D}_{\psi}^{+}$is a vector bundle on $[0,1] \times K$ and the parallel transport (for any $\mathcal{C}^{\infty}$ connexion on it) along $[0,1]$ yields a homotopy class of $\mathcal{C}^{\infty}$ isomorphism between vector bundles on $K$ :

$$
f_{K}: \operatorname{Ker} \mathcal{D}_{0, \psi}^{+} \longrightarrow \operatorname{Ker} \mathcal{D}_{1, \psi}^{+} .
$$

One can modify $\psi$ on $\left[0, \frac{\varepsilon}{2}\right) \times Y$ and $\left(1-\frac{\varepsilon}{2}, 1\right] \times Y$ such that for any $v \in \mathbb{C}^{N}$, $\left.\psi(v)\right|_{\{0\} \times Y}$ and $\left.\psi(v)\right|_{\{1\} \times Y}$ vanish, without altering the surjectivity property of $\mathcal{D}_{\psi}^{+}$. Then by Hodge theory:

$$
\left.\operatorname{Ker} \mathcal{D}_{0, \psi}^{+} \cong\left(\pi_{*} A_{0} \oplus \mathbb{C}^{N}\right)\right|_{K},\left.\quad \operatorname{Ker} \mathcal{D}_{1, \psi}^{+} \cong\left(\pi_{*} A_{1} \oplus \mathbb{C}^{N}\right)\right|_{K}
$$

Set then on $K$ :

$$
\pi_{*}\left(A_{0}, A_{1}, g\right):=\left(\pi_{*} A_{0} \oplus \mathbb{C}^{N}, \pi_{*} A_{1} \oplus \mathbb{C}^{N}, f_{K}\right) \in K_{0}^{\mathrm{rel}}(K) .
$$

The independence on the choice of $N$ and $\psi$ comes from the exact sequence (cf. [3], proof of Prop. 9.31):

$$
0 \rightarrow \operatorname{Ker} \mathcal{D}_{\psi_{1}}^{+} \longrightarrow \operatorname{Ker} \mathcal{D}_{\psi_{1}+\psi_{2}}^{+} \longrightarrow \mathbb{C}^{N_{2}} \rightarrow 0
$$

which yields the (topological) commutative diagram:

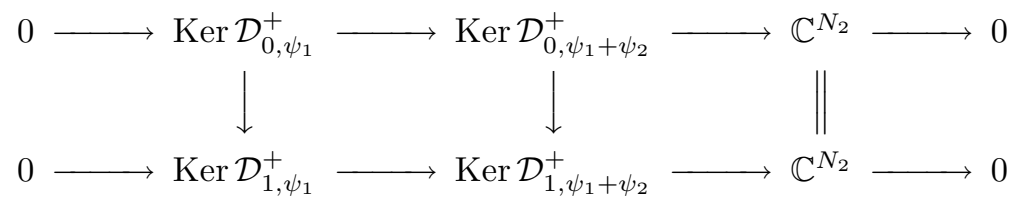

the same holding for $\psi_{2}$ and $N_{1}$ with the same isotopy class of $\mathcal{C}^{\infty}$ isomorphism $\operatorname{Ker} \mathcal{D}_{0, \psi_{1}+\psi_{2}}^{+} \stackrel{\sim}{\longrightarrow} \operatorname{Ker} \mathcal{D}_{1, \psi_{1}+\psi_{2}}^{+}$.

The independence on the choice of the metrics $h_{0}$ and $\omega$ and of the connexions $\nabla_{t}$ can be proved as follows: let $h_{1}, \omega^{\prime}$ and $\nabla_{t}^{\prime}$ be another choice and $\left(h_{s}, \omega_{s}, \nabla_{t, s}\right)_{s \in[0,1]}$ be a $\mathcal{C}^{\infty}$ family of metrics and connexions interpolating between $\left(h_{0}, \omega, \nabla_{t}\right)$ and $\left(h_{1}, \omega^{\prime}, \nabla_{t}^{\prime}\right)$. One can then perform the preceding construction on $[0,1] \times[0,1] \times K$ to obtain a family of Dirac operators $\mathcal{D}_{t, s, y, \psi}$. For any $s \in[0,1]$, one has $\operatorname{Ker} \mathcal{D}_{0, s, \psi}^{+} \cong\left(\pi_{*} A_{0} \oplus \mathbb{C}^{N}\right)$ and this isomorphism is obviously continuous since it is provided by Hodge theory. Thus, the parallel transport $g_{0}$ along $(t=0, s \in[0,1])$ is obviously isotopic to some holomorphic isomorphism (corresponding to the identity of $\pi_{*} A_{0} \oplus \mathbb{C}^{N}$ ). Accordingly, the

TOME $130-2002-\mathrm{N}^{\mathrm{O}} 2$ 
parallel transport $g_{1}$ along $(t=1, s \in[0,1])$ is isotopic to some holomorphic isomorphism between $\operatorname{Ker} \mathcal{D}_{1,0, \psi}$ and $\operatorname{Ker} \mathcal{D}_{1,1, \psi}$. One concludes using properties (i), (v) and (vi) and the fact that $f_{K 1}$ is isotopic to $g_{1} \circ f_{K 0} \circ g_{0}^{-1}$ (with obvious notation).

If $Y$ is compact, we are done. If $Y$ is quasiprojective, $\pi_{*} A_{0}$ and $\pi_{*} A_{1}$ are algebraic vector bundles on $Y$ and there exists some compact set $K \subset Y$ which is a retract of $Y$ (take $K=\bar{Y} \backslash \mathcal{V}$ where $\mathcal{V}$ is a sufficiently small tubular neighborhood of the divisor at infinity $\bar{Y} \backslash Y$ in some compactification $\bar{Y}$ of $Y$ ). Thus the $\mathcal{C}^{\infty}$ isomorphism $f_{K}$ produces a unique isotopy class of $\mathcal{C}^{\infty}$ isomorphisms between $\pi_{*} A_{0} \oplus \mathbb{C}^{N}$ and $\pi_{*} A_{1} \oplus \mathbb{C}^{N}$ on the whole $Y$.

Proposition 6.4. - This construction produces a direct image morphism $\pi_{*}: K_{0}^{\mathrm{rel}}(X) \rightarrow K_{0}^{\mathrm{rel}}(Y)$ which enters in the following commutative diagram:

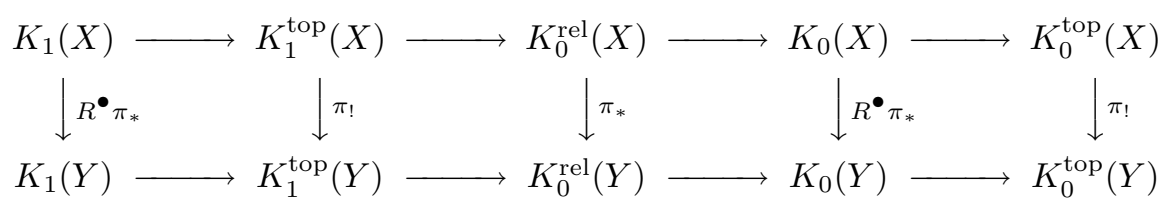

where $\pi$ ! are the direct image morphisms in topological $K$-theory constructed by Atiyah and Singer (for the $\operatorname{Spin}^{c}$ Dirac operator cf. [2]).

Proof. — If $(E, F, f) \in K_{0}^{\mathrm{rel}}(X)$ has a resolution to the right $\left(A_{E}^{\bullet}, A_{F}^{\bullet}, g^{\bullet}\right)$, one sets of course:

$$
\pi_{*}(E, F, f)=\sum_{k \geq 0}(-1)^{k} \pi_{*}\left(A_{E}^{k}, A_{F}^{k}, g^{k}\right) \in K_{0}^{\mathrm{rel}}(Y) .
$$

Consider $\mathcal{C}^{\infty}$ isomorphic acyclic bundles $\left(A_{0}, A_{1}, f\right)$ and $\left(A_{2}, A_{3}, g\right)$ then clearly in $K_{0}^{\mathrm{rel}}(Y)$ :

$$
\pi_{*}\left(A_{0}, A_{1}, f\right)+\pi_{*}\left(A_{2}, A_{3}, g\right)=\pi_{*}\left(A_{0} \oplus A_{2}, A_{1} \oplus A_{3}, f \oplus g\right) .
$$

If now $\left(A_{0}, A_{1}, f_{s}\right)_{s \in[0,1]}$ is an isotopy of $\mathcal{C}^{\infty}$ isomorphisms between acyclic bundles, the same kind of construction as for proving the independence on the metrics and connexions provides that the isotopy classes of $\mathcal{C}^{\infty}$ isomorphisms obtained from the $f_{s}$ between $\pi_{*} A_{0} \oplus \mathbb{C}^{N}$ and $\pi_{*} A_{1} \oplus \mathbb{C}^{N}$ are the same. By reasoning as in Proposition 2.1 this also shows that in $K_{0}^{\mathrm{rel}}(Y)$

$$
\begin{gathered}
\pi_{*}\left(A_{0}, A_{1}, f\right)=-\pi_{*}\left(A_{1}, A_{0}, f^{-1}\right) \text { and } \\
\pi_{*}\left(A_{0}, A_{1}, f\right)+\pi_{*}\left(A_{1}, A_{2}, g\right)=\pi_{*}\left(A_{0}, A_{2}, g \circ f\right) .
\end{gathered}
$$

Consider now some exact sequence

$$
0 \rightarrow A^{\prime} \stackrel{i}{\longrightarrow} A \stackrel{p}{\longrightarrow} A^{\prime \prime} \rightarrow 0
$$

of acyclic bundles on $X$ and a $\mathcal{C}^{\infty}$ left inverse $s: A \rightarrow A^{\prime}$ of $i$. Let $\sigma_{1}$ and $\sigma_{0}$ be the two sections of $\mathcal{O}(1)$ on $\mathbb{P}^{1}$ vanishing at 1 and 0 respectively, consider 
the pull-backs of $A^{\prime}$ and $A^{\prime} \oplus A$ on $\mathbb{P}^{1} \times X$ and the quotient $Q$ of the following monomorphism on $\mathbb{P}^{1} \times X$ :

$$
\sigma_{1} \otimes \operatorname{Id}_{A^{\prime}} \oplus \sigma_{0} \otimes i: A^{\prime} \longrightarrow \mathcal{O}(1) \otimes\left(A^{\prime} \oplus A\right) .
$$

Then for any $z \neq 1$ in $\mathbb{P}^{1},\left.Q\right|_{\{z\} \times X}$ is isomorphic to $A$ and $\left.Q\right|_{\{1\} \times X}$ is isomorphic to $A^{\prime} \oplus A^{\prime \prime}$. Choose any simple path $\gamma$ on $\mathbb{P}^{1}$ from 0 to 1 , then the parallel transport on $\left.Q\right|_{\gamma([0,1])}$ along $\gamma$ lies in the same isotopy class as $p \oplus s$. Take the direct image $\pi_{*} Q$ on $\mathbb{P}^{1} \times Y$ and restrict it to $\gamma([0,1]) \times Y$ then $\left.\pi_{*} Q\right|_{\{0\} \times X} \cong \pi_{*} A$ and $\left.\pi_{*} Q\right|_{\{1\} \times X} \cong \pi_{*} A^{\prime} \oplus \pi_{*} A^{\prime \prime}$ and $\pi_{*}\left(A, A^{\prime} \oplus A^{\prime \prime}, p \oplus s\right)=\left(\pi_{*} A, \pi_{*} A^{\prime} \oplus \pi_{*} A^{\prime \prime}, f\right)$ where $f$ is given by parallel transport on $\pi_{*} Q$ along $\gamma$, which in fact lies in the same isotopy class as $\pi_{*} p \oplus \sigma_{\pi}$ where

$$
0 \rightarrow \pi_{*} A^{\prime} \stackrel{\pi_{*} i}{\longrightarrow} \pi_{*} A \stackrel{\pi_{*} p}{\longrightarrow} \pi_{*} A^{\prime \prime} \rightarrow 0
$$

is the 'long' exact sequence associated to (34) and $\sigma_{\pi}: \pi_{*} A \rightarrow \pi_{*} A^{\prime}$ is any $\mathcal{C}^{\infty}$ left inverse of $\pi_{*} i$.

If $(E, F, f) \in K_{0}^{\mathrm{rel}}(X)$ has two resolutions to the right $\left(A_{E}^{\prime \bullet}, A_{F}^{\prime \bullet}, g^{\prime \bullet}\right)$ and $\left(A_{E}^{\prime \prime} \bullet, A_{F}^{\prime \prime}, g^{\prime \prime \bullet}\right)$, construct the dominating one $\left(A_{E}^{\bullet}, A_{F}^{\bullet}, g^{\bullet}\right)$ as in $(33)$, then there is a commutative diagram with (graded) exact lines:

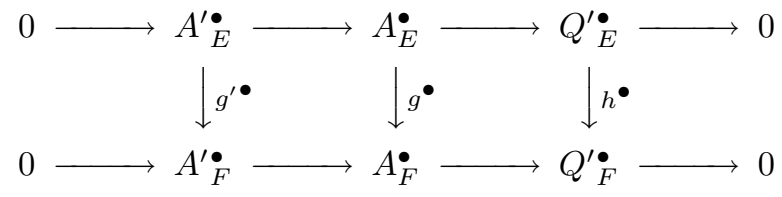

where the $Q_{E}^{\prime i}$ and $Q_{F}^{\prime i}$ are all acyclic, $h^{\bullet}$ are $\mathcal{C}^{\infty}$ (graded) isomorphisms and

$$
0 \rightarrow Q_{E}^{\prime 0} \longrightarrow Q_{E}^{\prime 1} \longrightarrow \cdots \longrightarrow Q_{E}^{m} \rightarrow 0
$$

is exact (and the same holds for the $Q_{E}^{\prime \prime} \bullet$, the $Q_{F}^{\prime \bullet}$ and the $Q_{F}^{\prime \prime} \bullet$ ). Thus to show that the image of $(E, F, f)$ in $K_{0}^{\mathrm{rel}}(Y)$ is independent on the choice of the resolution, it suffices to prove that for a commutative diagram with exact lines and in which all bundles are acyclic like

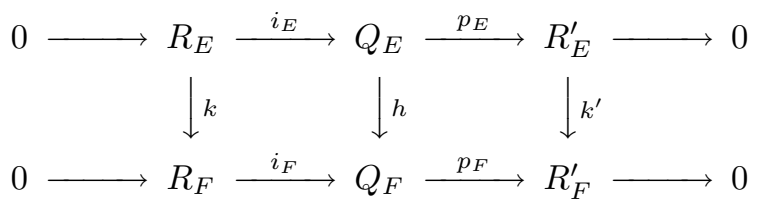

then $\pi_{*}\left(Q_{E}, Q_{F}, h\right)-\pi_{*}\left(R_{E}, R_{F}, k\right)-\pi_{*}\left(R_{E}^{\prime}, R_{F}^{\prime}, k^{\prime}\right)$ vanishes in $K_{0}^{\mathrm{rel}}(Y)$. But from previous considerations:

$$
\begin{aligned}
& \pi_{*}\left(Q_{E}, Q_{F}, h\right)=\pi_{*}\left(Q_{E}, R_{E} \oplus R_{E}^{\prime}, p_{E} \oplus s_{E}\right) \\
& \quad+\pi_{*}\left(R_{E} \oplus R_{E}^{\prime}, R_{F} \oplus R_{F}^{\prime}, k \oplus k^{\prime}\right)+\pi_{*}\left(R_{F} \oplus R_{F}^{\prime}, Q_{F},\left(p_{F} \oplus s_{F}\right)^{-1}\right)
\end{aligned}
$$

where $s_{E}: Q_{E} \rightarrow R_{E}$ and $s_{F}: Q_{F} \rightarrow R_{F}$ are $\mathcal{C}^{\infty}$ left inverses of $i_{E}$ and $i_{F}$. This is because the diagram commutes. The first and last terms vanish in $K_{0}^{\mathrm{rel}}(Y)$,

TOME $130-2002-\mathrm{N}^{\mathrm{O}} 2$ 
and the second one equals $\pi_{*}\left(R_{E}, R_{F}, k\right)+\pi_{*}\left(R_{E}^{\prime}, R_{F}^{\prime}, k^{\prime}\right)$. This proves the desired assertion.

Zero elements in $K_{0}^{\text {rel }}(X)$ coming from relations (i), (ii), (iii), and (iv) defining $K_{0}^{\text {rel }}$ are then easily seen to be sent by $\pi_{*}$ to zero in $K_{0}^{\text {rel }}(Y)$ from Lemma 6.1 and the above considerations.

Finally, the compatibility of $\pi_{*}$ on $K_{0}^{\text {rel }}$ and $R^{\bullet} \pi_{*}$ on $K_{0}$ is obvious, the

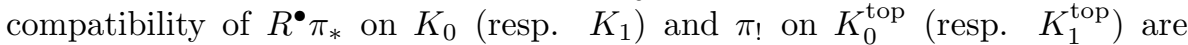
classical (see [2]); the resolution result (Corollary 6.3) shows that the image in $K_{0}^{\mathrm{rel}}(X)$ of any element of $K_{1}^{\mathrm{top}}(X)$ can be represented as $(A, A, f)$ where $A$ is acyclic. One can then calculate $\pi_{*}(A, A, f)$ as before using $[0,1] \times X$ or $S^{1} \times X$ indifferently. The compatibility of $\pi_{*}$ on $K_{0}^{\text {rel }}$ and $\pi_{\text {! on }} K_{1}^{\text {top }}$ follows then from the identification $K_{1}^{\text {top }}(X) \cong \operatorname{Ker}\left(K_{0}^{\text {top }}\left(S^{1} \times X\right) \rightarrow K_{0}^{\text {top }}(X)\right)$ and the compatibility of the contruction of [3] with the direct image in $K_{0}^{\text {top }}$ (see [3], Prop. 9.31 and Def. 9.32).

REMARK. - All this can be realized without $X$ nor $Y$ being projective provided the fibers of $\pi: X \rightarrow Y$ are projective and there exists a line bundle on $X$ whose restrictions to the fibers of $\pi$ are ample.

6.3. Riemann-Roch on $K_{0}^{\text {rel }}(X)$ in the case of a proper submersion. - Consider as above the vector bundle $A_{0}$ on $X$ with connexions $\left(\nabla_{t}\right)_{t \in[0,1]}$. They are supposed to respect some fixed hermitian metric $h_{0}$ on $A_{0}$.

Let $p_{2}:[0,1] \times X \rightarrow X$ be the projection on the second factor, and put on $p_{2}^{*} A_{0}$ the connexion

$$
\nabla_{A}=\mathrm{d} t \wedge \frac{\partial}{\partial t}+\nabla_{t}
$$

from which a Chern-Weil representative $\operatorname{ch}\left(\nabla_{A}^{2}\right)$ of the Chern character of $p_{2}^{*} A_{0}$ on $[0,1] \times X$ is constructed. Call $T X / Y$ the vertical tangent bundle of the fibration $\pi: X \rightarrow Y$ and $\operatorname{Td}(T X / Y)$ the Chern-Weil representative of its Todd class calculated with its hermitian compatible connexion for the Kähler metric $\omega$ on $X$. A consequence of Atiyah and Singer's families index theorem [2] is that the differential form

$$
N+\int_{X / Y} \operatorname{Td}(T X / Y) \wedge \operatorname{ch}\left(\nabla_{A}^{2}\right)
$$

on $[0,1] \times Y$ is cohomologous to the Chern character of the bundle $\operatorname{Ker} \mathcal{D}_{\psi}^{+}$on $[0,1] \times Y$ (here $\int_{X / Y}$ means partial integration on the fibers of $\pi$ and $N$ stands for the $\mathbb{C}^{N}$ part).

Choose any connexion $\nabla_{\psi}$ on $\operatorname{Ker} \mathcal{D}_{\psi}^{+}$whose restrictions to $\{i\} \times Y$ is trivial on the factor $\mathbb{C}^{N}$ and compatible with the holomorphic structures of $\pi_{*} A_{i}$ (for $i=0$ and 1 and modulo the isomorphisms $\left.\operatorname{Ker} \mathcal{D}_{\psi}^{+}\right|_{\{i\} \times Y} \cong \pi_{*} A_{i} \otimes \mathbb{C}^{N}$ ). 
The preceding assertion yields that there exists an odd differential form $\alpha$ on $[0,1] \times Y$ such that

$$
\mathrm{d} \alpha=\operatorname{ch}\left(\nabla_{\psi}^{2}\right)-\left(N+\int_{X / Y} \operatorname{Td}(T X / Y) \wedge \operatorname{ch}\left(\nabla_{A}^{2}\right)\right) .
$$

$\operatorname{Td}(T X / Y)$ being independent on $t \in[0,1]$, one can then integrate along $[0,1]$ to obtain:

$$
\begin{aligned}
\int_{[0,1]} \operatorname{ch}\left(\nabla_{\psi}^{2}\right)-\int_{[0,1]}\left(N+\int_{X / Y} \operatorname{Td}(T X / Y) \wedge \operatorname{ch}\left(\nabla_{A}^{2}\right)\right) \\
=\overline{\operatorname{ch}}\left(\nabla_{0, \psi}, \nabla_{1, \psi}\right)-\int_{X / Y} \operatorname{Td}(T X / Y) \wedge \overline{\operatorname{ch}}\left(\nabla_{0}, \nabla_{1}\right) \\
=\int_{[0,1]} \mathrm{d} \alpha=\int_{[0,1]}\left(d_{[0,1]} \alpha+d_{Y} \alpha\right)=\mathrm{d}_{Y}\left(\int_{[0,1]} \alpha\right)+\alpha_{1}-\alpha_{0}
\end{aligned}
$$

where the $\nabla_{t, \psi}$ are the restrictions of $\nabla_{\psi}$ to $\left.\operatorname{Ker} \mathcal{D}_{\psi}^{+}\right|_{\{t\} \times Y}$ on $Y, \mathrm{~d}_{[0,1]}$ and $\mathrm{d}_{Y}$ stand for the exterior differential operators along $[0,1]$ and $Y$ and $\alpha_{0}$ and $\alpha_{1}$ are the restrictions of $\alpha$ to $\{0\} \times Y$ and $\{1\} \times Y$ respectively.

THEOREM 6.5. - If $X$ and $Y$ are (compact) projective, there exists such a connexion $\nabla_{\psi}$ and such a form $\alpha$ which verify that $\alpha_{0}$ and $\alpha_{1}$ belong to $\bigoplus_{p} F_{p} \wedge^{2 p-1}(Y)+\mathrm{d} \wedge(Y)$.

Corollary. - If $X$ and $Y$ are projective, the cohomology classes of $\overline{\operatorname{ch}}\left(\nabla_{0, \psi}, \nabla_{1, \psi}\right)$ and $\int_{X / Y} \operatorname{Td}(T X / Y) \wedge \overline{\operatorname{ch}}\left(\nabla_{0}, \nabla_{1}\right)$ coincide in $H^{2 \bullet-1}\left(\wedge(Y) / F_{\bullet}\right)$.

Corollary 6.6 (Riemann-Roch theorem on $K_{0}^{\text {rel }}$ for proper submersions.)

If $X$ and $Y$ are projective, then for any $(E, F, f) \in K_{0}^{\mathrm{rel}}(X)$

$$
\mathcal{N}_{\text {ch }}\left(\pi_{*}(E, F, f)\right)=\int_{X / Y} \operatorname{Td}(T X / Y) \wedge \mathcal{N}_{\text {ch }}(E, F, f) .
$$

Proof of corollary. - Note that $H^{2 \bullet-1}\left(\wedge(X) / F_{\bullet}\right)$ is naturally a module on $\bigoplus_{p} H^{p, p}(X, \mathbb{C})$ and that partial integration on the fibres of $\pi$ gives a natural map from $H^{2 \bullet-1}\left(\wedge(X) / F_{\bullet}\right)$ to $H^{2 \bullet-1}\left(\wedge(Y) / F_{\bullet}\right)$. Then $\nabla_{t}, \psi$ and $\nabla_{\psi}$ have been chosen such that $\overline{c h}\left(\nabla_{0}, \nabla_{1}\right)$ and $\overline{c h}\left(\nabla_{0, \psi}, \nabla_{1, \psi}\right)$ are representatives of $\mathcal{N}_{\mathrm{ch}}\left(A_{0}, A_{1}, g\right)$ and $\mathcal{N}_{\mathrm{ch}}\left(\pi_{*}\left(A_{0}, A_{1}, g\right)\right)$ respectively so that the second corollary is true for $\mathcal{C}^{\infty}$ isomorphic acyclic bundles. Using resolutions (Corollary 6.3) yields the full result.

6.4. Proof of theorem 6.5. - The Kähler metric $\omega$ on $X$ and some hermitian metric $h_{0}$ on $A_{0}$ being fixed, and $\psi$ being chosen as in the preceding subsection, consider the compatible hermitian connexion on $T X / Y$ and its pull-back on $[0,1] \times X$ by $p_{2}$, and the connexion $\nabla_{A}$ on $A_{0}$ (35). Denote by $\nabla$ the induced connexion on the bundle $\wedge^{0} \bullet X / Y \otimes A_{0}$ of vertical differential forms of complex types $(0, \bullet)$ with values in $A_{0}$ over $[0,1] \times X$. $\mathcal{E}^{+}$and $\mathcal{E}^{-}$

TOME $130-2002-\mathrm{N}^{\mathrm{O}} 2$ 
are seen as infinite dimensional $\mathcal{C}^{\infty}$ vector bundles on $[0,1] \times Y$ and the Dirac operator $\mathcal{D}=\mathcal{D}_{t, y}$ as a bundle morphism $\mathcal{E}_{y}^{+} \oplus \mathcal{E}_{y}^{-} \rightarrow \mathcal{E}_{y}^{-} \oplus \mathcal{E}_{y}^{+}$. For any vector field $U$ tangent to $[0,1] \times Y$, let $U_{H}$ be the vector field on $[0,1] \times X$ such that $\pi_{*} U_{H}=U$ and $U_{H}$ is pointwise orthogonal to $T X / Y$. Endow $\mathcal{E}^{+}$and $\mathcal{E}^{-}$with the connexion $\widetilde{\nabla}$ defined by

$$
\widetilde{\nabla}_{U}(s)=\nabla_{U_{H}} s
$$

$\left(s\right.$ is any element of $\mathcal{C}^{\infty}\left([0,1] \times Y, \mathcal{E}^{+} \oplus \mathcal{E}^{-}\right) \cong \mathcal{C}^{\infty}\left([0,1] \times X, \wedge^{\left.0, \bullet X / Y \otimes A_{0}\right)}\right)$. This connexion can be shown to respect the $L^{2}$ inner product on $\mathcal{E}^{+}$and $\mathcal{E}^{-}$as in [7], Thm. 1.14 and [3], Prop. 9.13. Moreover, consider some vertical tangent vector $v \in T X / Y$ decomposed as the sum of its holomorphic and antiholomorphic parts: $\quad v=v^{(1,0)}+v^{(0,1)}$. Call $v^{(1,0) *}$ the one form dual to $v^{(1,0)}$ throw the Kähler metric on $T X / Y$ and consider the Clifford action of $T X / Y$ on $\wedge^{0, \bullet} X / Y \otimes A_{0}$ defined by

$$
c(v)=\left(\sqrt{2} v^{(1,0) *} \wedge\right)-\left(\sqrt{2} \iota_{v^{(0,1)}}\right)
$$

( $\iota$ being interior product), finally let $\pi_{X / Y}$ be the orthogonal projection of $T([0,1] \times X)$ on $T X / Y$. Set then:

$$
\begin{aligned}
T: T([0,1] \times Y) \times T([0,1] \times Y) & \longrightarrow T X / Y \\
(v, w) & \longmapsto \pi_{X / Y}\left[v_{H}, w_{H}\right],
\end{aligned}
$$

where $\left[v_{H}, w_{H}\right]$ is the Lie bracket of vector fields. The Bismut superconnexion on $\mathcal{E}^{+} \oplus \mathcal{E}^{-}$(which Bismut calls "Levi-Civita superconnexion") is defined to be (cf. [9], Def. 1.7):

$$
B_{u}=\widetilde{\nabla}+\sqrt{u} \mathcal{D}-\frac{1}{2 \sqrt{2 u}} c(T) .
$$

Note that there is a factor $\sqrt{2}$ between [9], Def. 1.7 and [7], Def. 2.1. Consider the trivial bundle $\mathbb{C}^{N}$ on $[0,1] \times Y$ with trivial canonical hermitian metric and trivial connexion, call again $\widetilde{\nabla}$ the obviously associated connexion on $\mathcal{E}^{+} \oplus \mathbb{C}^{N} \oplus$ $\mathcal{E}^{-}$and take some $\mathcal{C}^{\infty}$ increasing function $\varphi$ on $[0,+\infty)$ vanishing on $[0,1]$ and which equals 1 on $[2,+\infty)$. Set then

$$
C_{u}=\widetilde{\nabla}+\sqrt{u} \mathcal{D}_{\varphi(u) \psi}-\frac{1}{2 \sqrt{2 u}} c(T)
$$

where $\mathcal{D}_{\varphi(u) \psi}$ is associated to the map $\varphi(u) \psi: \mathbb{C}^{N} \rightarrow \mathcal{E}^{-}$. This is a superconnexion on $\left(\mathcal{E}^{+} \oplus \mathbb{C}^{N}\right) \oplus \mathcal{E}^{-}$. Denote by $\pi_{\psi}$ the orthogonal projection on the subbundle $\operatorname{Ker} \mathcal{D}_{\psi}^{+} \subset \mathcal{E}^{+} \oplus \mathbb{C}^{N}$, then the connexion $\nabla_{\psi}=\pi_{\psi} \widetilde{\nabla} \pi_{\psi}$ has the properties stated in the preceding subsection because of the choice of $\psi$ and of [9], Thm. 3.2. Moreover, $\nabla_{\psi}$ respects the hermitian metric on $\operatorname{Ker} \mathcal{D}_{\psi}^{+}$induced by restriction of the direct sum of the $L^{2}$ inner product on $\mathcal{E}^{+}$and the canonical hermitian product on $\mathbb{C}^{N}$ (see [3], Prop. 9.13).

BULletin DE LA SOCiÉtÉ MATHÉmATIQUE DE FRANCE 
Finally, denote by $\operatorname{Tr}_{s}$ the supertrace on $\left(\mathcal{E}^{+} \oplus \mathbb{C}^{N}\right) \oplus \mathcal{E}^{-}$(which is the trace on $\mathcal{E}^{+} \oplus \mathbb{C}^{N}$ minus the trace on $\mathcal{E}^{-}$) and by $\phi$ the linear operator on $\wedge^{\text {even }}([0,1] \times Y)$ which divides by $(2 \pi i)^{p}$ the forms of degree $2 p$. Then it follows from [3], Thm. 9.23 that as u tends to $+\infty$ :

$$
\phi \operatorname{Tr}_{s}\left(\exp -C_{u}^{2}\right) \longrightarrow \phi \operatorname{Tr}_{s}\left(\exp -\nabla_{\psi}^{2}\right)
$$

On the other hand, one has for $u \leq 1$ :

$$
\phi \operatorname{Tr}_{s}\left(\exp -C_{u}^{2}\right)=\phi \operatorname{Tr}_{s}\left(\exp -B_{u}^{2}\right)+N
$$

Lemma 6.7. - As u tends to zero:

$$
\phi \operatorname{Tr}_{s}\left(\exp -C_{u}^{2}\right)=N+\int_{X / Y} \operatorname{Td}(T X / Y) \wedge \operatorname{ch}\left(\nabla_{A}^{2}\right)+\mathcal{O}(u) .
$$

Proof. — This is of course not a direct application of [7], Thm. 2.2 because of the presence of $[0,1]$ and the fact that $\nabla_{A}$ is not holomorphic on $A_{0}$ restricted to $\{t\} \times X / Y$. In particular no information on the complex types of the differential forms involved is available here.

However, the proof of [7], Thm. 2.2, refers to a previous work of Bismut [4] which is not stated in the holomorphic context: the complex structure of the fibres is only involved throw the induced $\operatorname{Spin}^{c}$ structure; the fact that $X$ is Kähler and that its Kähler metric is independent of $t \in[0,1]$ (this implies the Kähler condition of [9], $\S \mathrm{I}$ a)) ensures that the connexion on $\wedge^{0, \bullet} T X / Y$ is a suitable Clifford connexion in the sense of [3] $\S \S 10.1$ and 10.2.

Finally the only condition on the "twisting" bundle $A_{0}$ is that its connexion be compatible with its hermitian metric, which is here satisfied by hypothesis; thus the families index theorem [3], Thm. 10.23 can be applied and gives the desired formula from a standard calculation (see [3], Thm. 4.9 or [7], formula (2.12) in the proof of Theorem 2.2).

Afterwards, one calculates as in [7], Thm. 2.9:

$$
\begin{aligned}
\frac{\partial}{\partial u} \operatorname{Tr}_{s}\left(\exp -C_{u}^{2}\right)=-\mathrm{d}_{\operatorname{Tr}_{s}}\left[\left(\frac{\partial C_{u}}{\partial u}\right) \exp -C_{u}^{2}\right] \\
=\frac{-1}{2 u} \mathrm{~d}_{\operatorname{Tr}_{s}}\left(\left[\sqrt{u} \mathcal{D}_{\varphi(u) \psi}+2 u \frac{\partial \mathcal{D}_{\varphi(u) \psi}}{\partial u}+\frac{c(T)}{2 \sqrt{2 u}}\right] \exp -C_{u}^{2}\right) .
\end{aligned}
$$

It follows then from [3], Thm. 9.23 that

$$
\operatorname{Tr}_{s}\left(\frac{\partial C_{u}}{\partial u} \exp -C_{u}^{2}\right)=\mathcal{O}\left(u^{-\frac{3}{2}}\right) \quad \text { as } \quad u \rightarrow+\infty
$$

with respect to each $\mathcal{C}^{\ell}$ norm on $[0,1] \times Y$, and from [22], Thm. 1.6.1 and [7], Thm. 2.11 that there is an asymptotic expansion for each $k$ as $u$ tends to 0 :

$$
\operatorname{Tr}_{s}\left(\frac{\partial C_{u}}{\partial u} \exp -C_{u}^{2}\right)=\sum_{j=-\operatorname{dim} X / Y-1}^{k-1} D_{j} u^{j}+\mathcal{O}\left(u^{k}\right)
$$

TOME $130-2002-\mathrm{N}^{\mathrm{O}} 2$ 
where $\mathcal{O}\left(u^{k}\right)$ is uniform on $[0,1] \times Y$ for any $\mathcal{C}^{\ell}$ norm on $[0,1] \times Y$. It follows from (38) and Lemma 6.7 that $D_{j}$ is a closed form on $[0,1] \times Y$ for any $j \leq-2$ : that $D_{j}$ vanishes or not for $j \leq-3$ (as in [7], Thm. 2.11) does not matter here. Choose some square root $\sqrt{2 \pi i}$ of $2 \pi i$ in $\mathbb{C}$ and extend $\phi$ to $\wedge^{\text {odd }}([0,1] \times Y)$ by dividing $k$-forms by $\sqrt{2 \pi i}^{k}$ and set

$$
\begin{aligned}
& \zeta^{3}(s)=-\frac{1}{\Gamma(s)} \int_{1}^{+\infty} u^{s} \phi \operatorname{Tr}_{s}\left(\frac{\partial C_{u}}{\partial u} \exp -C_{u}^{2}\right) \mathrm{d} u, \\
& \zeta^{4}(s)=-\frac{1}{\Gamma(s)} \int_{0}^{1} u^{s} \phi \operatorname{Tr}_{s}\left(\frac{\partial C_{u}}{\partial u} \exp -C_{u}^{2}\right) \mathrm{d} u .
\end{aligned}
$$

$\zeta^{3}$ is a holomorphic function for $\operatorname{Re}(s)<\frac{1}{2}$ whereas $\zeta^{4}$ is holomorphic for $\operatorname{Re}(s)>\operatorname{dim} X / Y+1$ and because of (39) extends to a meromorphic function on $\mathbb{C}$ which is holomorphic for $|s|<\frac{1}{2}$. Put

$$
\begin{gathered}
\alpha=\zeta^{3 \prime}(0)+\zeta^{4 \prime}(0) \\
=-\int_{1}^{+\infty} \frac{1}{2} \phi \operatorname{Tr}_{s}\left(\left[\sqrt{u} \mathcal{D}_{\varphi(u) \psi}+2 u \frac{\partial \mathcal{D}_{\varphi(u) \psi}}{\partial u}+\frac{c(T)}{2 \sqrt{2 u}}\right] \exp -C_{u}^{2}\right) \frac{\mathrm{d} u}{u} \\
\quad-\int_{0}^{1}\left(\frac{1}{2} \phi \operatorname{Tr}_{s}\left(\left[\sqrt{u} \mathcal{D}_{\psi}+\frac{c(T)}{2 \sqrt{2 u}}\right] \exp -C_{u}^{2}\right)-\sum_{j \leq-1} D_{j} u^{j+1}\right) \frac{\mathrm{d} u}{u} \\
+\Gamma^{\prime}(1) D_{-1}-\sum_{j=-\operatorname{dim} X / Y-1}^{-2} \frac{D_{j}}{j+1} .
\end{gathered}
$$

From (38), (37) and Lemma 6.7, $\sqrt{2 \pi i} \alpha$ verifies the transgression formula (36). It remains to check that $\left.\alpha\right|_{\{0\} \times Y}$ and $\left.\alpha\right|_{\{1\} \times Y}$ lie in $\bigoplus_{p} F_{p} \wedge^{2 p-1}(Y)+\mathrm{d} \wedge(Y)$. But on $t=0$ and $t=1, \psi$ vanishes so that following [7], Thm. 2.9

$$
\operatorname{Tr}_{s}\left(\left[\sqrt{u} \mathcal{D}_{1}, \psi+\frac{c(T)}{2 \sqrt{2 u}}\right] \exp -C_{u}^{2}\right)=\frac{1}{2}(\partial-\bar{\partial}) \operatorname{Tr}_{s}\left(N_{u} \exp -B_{1}^{2}, u\right)
$$

is the so called first transgression form $\left(B_{1}, u\right.$ and $\mathcal{D}_{0}, \psi$ are the restrictions of $B_{u}$ and $\mathcal{D}_{\psi}$ to $\{0\} \times Y$ and $\{1\} \times Y$ respectively. ${ }_{1}^{0}$ means that the formulas are valid either for 0 or 1). Thus if one puts as in [9], Def. 3.7:

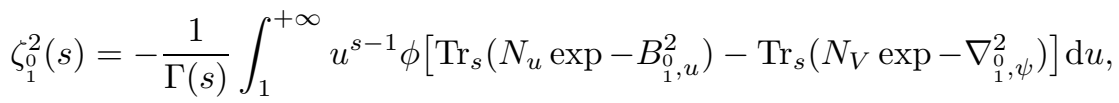

$$
\begin{aligned}
& \zeta_{1}^{1}(s)=-\frac{1}{\Gamma(s)} \int_{0}^{1} u^{s-1} \phi\left[\operatorname{Tr}_{s}\left(N_{u} \exp -B_{1}^{2}, u\right)-\operatorname{Tr}_{s}\left(N_{V} \exp -\nabla_{1}^{2}, \psi\right)\right] \mathrm{d} u, \\
& \zeta_{1}(s)=\zeta_{1}^{1}(s)+\zeta_{1}^{2}(s) \quad \text { (after suitable meromorphic continuation), } \\
& T_{0}\left(\omega, h_{0}\right)=\frac{\partial}{\partial s} \zeta_{1}(0),
\end{aligned}
$$

BULletin DE LA SOCiÉtÉ MATHÉmATiQUe DE FRANCE 
$\left(T_{0}\left(\omega, h_{0}\right)\right.$ is the so called higher analytic torsion form) one gets:

$$
\alpha_{1}=\frac{1}{2 \sqrt{2 \pi i}}(\partial-\bar{\partial}) T_{0}\left(\omega, h_{0}\right)=\frac{1}{\sqrt{2 \pi i}} \partial T_{1}\left(\omega, h_{0}\right)+\mathrm{d} \frac{-1}{2 \sqrt{2 \pi i}} T_{1}\left(\omega, h_{0}\right)
$$

but now $T_{0}\left(\omega, h_{0}\right)$ and $T_{1}\left(\omega, h_{0}\right)$ are sums of forms of type $(p, p)$ (see [9], Thm. 3.9: this is the important feature of $T_{0}\left(\omega, h_{0}\right)$, the precise definition of $N_{V}$ and $N_{u}$ is not needed here) so that $\alpha$ lies in $\bigoplus_{p} F_{p} \wedge^{2 p-1}(Y)+d \wedge(Y)$ as desired.

The theorem is proved.

REMARK 6.8. - In the quasiprojective case, one would need that $T_{0}\left(\omega, h_{0}\right)$ be logarithmic which is certainly false in general (its degree zero part is the analytic torsion of the fibres which is not bounded as shown by Bismut in [6]). Roessler has faced the same difficulty in his work on arithmetic Riemann-Roch on higher arithmetic $K$-theory (see [36], final remark).

6.5. Albanese map of moduli spaces of vector bundles over abelian surfaces. - Let $X$ be an abelian surface, it is of course isomorphic to its Albanese variety, and its Picard variety is isomorphic to its dual abelian variety $\widehat{X}$. For some moduli space $\mathcal{M}$ of vector bundles on $X$ and $E_{0} \in \mathcal{M}$. Yoshioka defines the map

$$
\begin{aligned}
\mathfrak{a} & =" \operatorname{det} " \times \alpha: \mathcal{M} \rightarrow \widehat{X} \times X, \\
" \operatorname{det} "(E) & =\operatorname{det}(E) \otimes\left(\operatorname{det}\left(E_{0}\right)\right)^{*}, \\
\alpha(E) & =\operatorname{det} R^{\bullet} p_{\hat{X} *}\left(\left(E-E_{0}\right) \otimes \mathcal{P}\right),
\end{aligned}
$$

where $\mathcal{P}$ is the Poincaré line bundle on $\widehat{X} \times X$ and $p_{\hat{X}}: \widehat{X} \times X \rightarrow \widehat{X}$ is the projection on the first factor ("det" $E$ (resp. $\alpha(E)$ ) is a flat line bundle on $X$ (resp. $\widehat{X})$ so that it naturally defines a class in $\widehat{X}($ resp. in $\widehat{\widehat{X}}=X)$ ). Then he shows that for most moduli spaces $\mathcal{M}$, this map $\mathfrak{a}$ is an Albanese map and that in some other cases, $\mathcal{M}$ is an abelian surface and $\mathfrak{a}$ is an immersion (see [38] and [37] for the precise statements).

Proposition. - For any $E \in \mathcal{M}$, one has in $\widehat{X} \times X \cong J_{1}(X) \oplus J_{2}(X)$ :

$$
\mathfrak{a}(E)=\mathcal{M}_{c_{\text {tot }}}\left([E]-\left[E_{0}\right]\right) \circ \varepsilon
$$

where $\varepsilon$ changes the sign in $\widehat{X}$ and leaves $X$ unchanged. (Here $[E]$ stands for the class of $E$ in $K_{0}(X)$ and one does not need to exhibit any $\mathcal{C}^{\infty}$ isomorphism because the class is taken modulo integral cohomology.)

Proof. - From Proposition 5.10 and Lemma 5.11, one has modulo integral cohomology

$$
" \operatorname{det} "(E)=-\mathcal{N}_{c_{1}}\left([E]-\left[E_{0}\right]\right)
$$

TOME $130-2002-\mathrm{N}^{\mathrm{O}} 2$ 
this proves the "first half" of the proposition. Accordingly modulo integral cohomology

$$
\begin{aligned}
\alpha(E) & =-\mathcal{N}_{c_{1}}\left(R^{\bullet} p_{\hat{X} *}\left([E \otimes \mathcal{P}]-\left[E_{0} \otimes \mathcal{P}\right]\right)\right) \\
& =-\mathcal{N}_{\operatorname{ch}_{1}}\left(p_{\hat{X} *}\left(E_{0} \otimes \mathcal{P}, E \otimes \mathcal{P}, f \otimes \operatorname{Id}_{\mathcal{P}}\right)\right)
\end{aligned}
$$

where $f$ is any $\mathcal{C}^{\infty}$ isomorphism between $E_{0}$ and $E$ (or between $E_{0} \oplus \mathbb{C}^{N}$ and $E \oplus \mathbb{C}^{N}$ : there exist always some such $N$ and $f$ because $E$ and $E_{0}$ have same class in $\left.K_{0}^{\mathrm{top}}(X)\right)$.

Now one can apply Corollary 6.6: the submersion is here $p_{\hat{X}}$ the vertical tangent bundle is the tangent bundle of $X$ which is flat so that

$$
\begin{aligned}
\mathcal{N}_{\mathrm{ch}}\left(p _ { \hat { X } * } \left(E_{0} \otimes \mathcal{P},\right.\right. & \left.\left.E \otimes \mathcal{P}, f \otimes \operatorname{Id}_{\mathcal{P}}\right)\right) \\
& =\int_{\hat{X} \times X / \hat{X}} \mathcal{N}_{\operatorname{ch}}\left(E_{0} \otimes \mathcal{P}, E \otimes \mathcal{P}, f \otimes \operatorname{Id}_{\mathcal{P}}\right) \\
& =\int_{\hat{X} \times X / \hat{X}} \mathcal{N}_{\operatorname{ch}}\left(E_{0}, E, f\right) \wedge \operatorname{ch} \mathcal{P} \\
& =\int_{\hat{X} \times X / \hat{X}} \mathcal{N}_{\text {ch }}\left(E_{0}, E, f\right) \wedge \exp \left(c_{1}(\mathcal{P})\right)
\end{aligned}
$$

(the second equality is due to Proposition 3.2). Let $V$ be the universal covering of $X$ and $\Lambda$ the lattice in $V$ such that $X=V / \Lambda$. Let $\widehat{V}=\bar{V}^{*}$ be the antidual space of $V$ and $\widehat{\Lambda}$ the dual lattice of $\Lambda$, then $\widehat{X}=\widehat{V} / \widehat{\Lambda}$ (see [33], p. 86). To any line bundle $L$ on an abelian variety is associated some hermitian form $H_{L}$ on the universal covering ([33], p. 20), and $c_{1}(L)=-\operatorname{Im} H_{L}$ as a real 2-form. The hermitian form associated to $\mathcal{P}$ on $X \times \widehat{X}$ is ([33], p. 86):

$$
H_{\mathcal{P}}\left(\left(v_{1}, \ell_{1}\right),\left(v_{2}, \ell_{2}\right)\right)=\overline{\ell_{2}\left(v_{1}\right)}+\ell_{1}\left(v_{2}\right)
$$

(for $\left.\left(v_{i}, \ell_{i}\right) \in V \times \widehat{V}\right)$. Thus for degree reasons, it follows from (41) and (42):

$$
\alpha(E)=\int_{\hat{X} \times X / \hat{X}} \mathcal{N}_{R \mathrm{ch}_{2}}\left(E_{0}, E, f\right) \wedge c_{1}(\mathcal{P})
$$

(because $R \mathrm{ch}_{2}=-\mathrm{ch}_{2}$ ). Finally $H^{1,2}(X)$ is isomorphic throw Poincaré duality to $\left(H^{0,1}(X)\right)^{*} \cong H^{0,1}(\widehat{X})$, which can be directly verified to be the same map as:

$$
\beta \in H^{1,2}(X) \longmapsto \int_{\hat{X} \times X / \hat{X}} \beta \wedge c_{1}(\mathcal{P}) \in H^{0,1}(\widehat{X})
$$

from (43) (some care has to be taken because of the various descriptions of $\widehat{X}$ (see the "experimental error" in [33], p. 87)). 


\subsection{Direct image for $K_{0}^{\text {def }}$ in the case of a closed immersion}

As usual a general Riemann-Roch theorem for projective manifolds will be obtained from the particular cases of a proper submersion and a closed immersion.

Consider then a closed immersion $\iota: Y \rightarrow X$ with $Y$ and $X$ projective. I won't construct here a direct image morphism from $K_{0}^{\mathrm{rel}}(Y)$ to $K_{0}^{\mathrm{rel}}(X)$ but only from $K_{0}^{\text {def }}(Y)$ to $K_{0}^{\operatorname{def}}(X)$, this is because I found no way to associate canonically to a $\mathcal{C}^{\infty}$ isomorphism between two bundles on $Y$ any "C ${ }^{\infty}$ isomorphism" between their resolutions by vector bundles on $X$.

Consider some smooth connected algebraic variety $\Sigma$ and some algebraic vector bundle $\eta$ on $\Sigma \times Y$, take two points $q_{0}$ and $q_{1} \in \Sigma$ and choose some path $\widetilde{q}:[0,1] \rightarrow \Sigma$ such that $\widetilde{q}(0)=q_{0}$ and $\widetilde{q}(1)=q_{1}$. Set $\eta_{0}=\left.\eta\right|_{\left\{q_{0}\right\} \times X}$ and $\eta_{1}=\left.\eta\right|_{\left\{q_{1}\right\} \times X}$ and consider the element $\left(\eta_{0}, \eta_{1}, f_{01}\right) \in K_{0}^{\text {rel }}(Y)$ where $f_{01}: \eta_{0} \stackrel{\sim}{\rightarrow} \eta_{1}$ stands for the isotopy class of $\mathcal{C}^{\infty}$ isomorphism obtained by parallel transport along $\widetilde{q}$ (for any connexion on $\eta$ ). $K_{0}^{\text {def }}(Y)$ is the subgroup of $K_{0}^{\mathrm{rel}}(Y)$ generated by such elements.

To construct the direct image of $\left(\eta_{0}, \eta_{1}, f_{01}\right)$ call again $\iota$ the obvious immersion $\Sigma \times Y \rightarrow \Sigma \times X$ and consider some left resolution on $\Sigma \times X$ of the sheaf $\iota_{*} \mathcal{O}_{\Sigma \times Y}(\eta)$ by vector bundles:

$$
0 \rightarrow \mathcal{O}_{\Sigma \times X}\left(\xi_{m}\right) \rightarrow \mathcal{O}_{\Sigma \times X}\left(\xi_{m-1}\right) \rightarrow \cdots \rightarrow \mathcal{O}_{\Sigma \times X}\left(\xi_{0}\right) \rightarrow \iota_{*} \mathcal{O}_{\Sigma \times Y}(\eta) \rightarrow 0 .
$$

For any $i$, set $\xi_{i 0}=\left.\xi_{i}\right|_{\left\{q_{0}\right\} \times X}, \xi_{i 1}=\left.\xi_{i}\right|_{\left\{q_{1}\right\} \times X}$ and call $g_{i 01}$ the isotopy class of $\mathcal{C}^{\infty}$ isomorphism between $\xi_{i 0}$ and $\xi_{i 1}$ obtained by parallel transport along $\widetilde{q}$. Set then:

$$
\iota_{*}\left(\eta_{0}, \eta_{1}, f_{01}\right)=\sum_{i=0}^{m}(-1)^{i}\left(\xi_{i 0}, \xi_{i 1}, g_{i 01}\right) .
$$

Proposition. - This yields a direct image morphism which fits in the following commutative diagram:

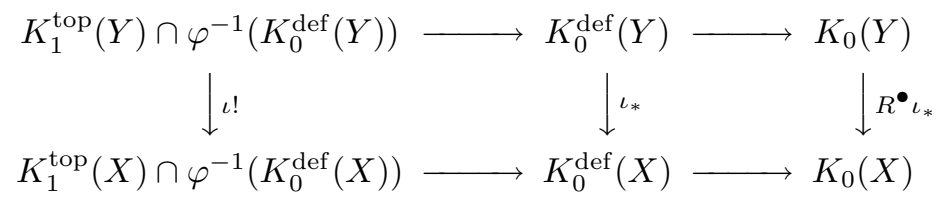

Proof. - Consider first the compatibility of $\iota_{*}$ with relation (ii) defining $K_{0}^{\text {rel }}$ : suppose $f_{01}: \eta_{0} \rightarrow \eta_{1}$ is isotopic to some holomorphic isomorphism $h_{\eta}: \eta_{0} \rightarrow \eta_{1}$ (which stands for the "equality" of $\eta_{0}$ and $\left.\eta_{1}\right)$. Then $\left(\xi_{\bullet 0}\right)$ and $\left(\xi_{\bullet 1}\right)$ provide resolutions of the same sheaf $\iota_{*} \mathcal{O}_{Y}\left(\eta_{0}\right) \stackrel{h_{\eta}}{\cong} \iota_{*} \mathcal{O}_{Y}\left(\eta_{1}\right)$. From [10], démonstration $\mathrm{du}$ lemme 11 , there exists a third resolution $\left(\xi_{\bullet 2}\right)$ with graded epimorphisms $\xi_{\bullet 2} \stackrel{h_{\bullet}}{\longrightarrow} \xi_{\bullet 0}$ and $\xi_{\bullet 2} \stackrel{k_{\bullet}}{\longrightarrow} \xi_{\bullet 1}$ whose kernels are exact sequences of vector bundles $\theta_{\bullet}$ and $\kappa_{\bullet}$. Moreover for any $0 \leq i \leq m, \theta_{i}$ and $\kappa_{i}$ have same class in

TOME $130-2002-\mathrm{N}^{\mathrm{O}} 2$ 
topological $K$-theory so that it is possible by a straightforward construction to find some (algebraic) exact sequence of trivial bundles $\left(\mathbb{C}^{N \bullet}\right)$ and $\mathcal{C}^{\infty}$ isomorphisms $j_{i}: \theta_{i} \oplus \mathbb{C}^{N_{i}} \stackrel{\sim}{\rightarrow} \kappa_{i} \oplus \mathbb{C}^{N_{i}}$ which commute with the morphisms of exact sequences $\left(\theta_{\bullet} \oplus \mathbb{C}^{N_{\bullet}}\right)$ and $\left(\kappa_{\bullet} \oplus \mathbb{C}^{N_{\bullet}}\right)$. Consider then $\zeta_{\bullet}:=\xi_{\bullet} \oplus \theta_{\bullet} \oplus \kappa_{\bullet} \oplus \mathbb{C}^{N_{\bullet}}$ on $\widetilde{q}([0,1]) \times X$ and identify $\zeta_{i 0}$ and $\zeta_{i 1}$ for any $i$ throw

$$
f_{i}:=\left(k_{i} \oplus \sigma_{i}\right) \circ\left(h_{i} \oplus s_{i}\right)^{-1} \oplus j_{i}^{-1}
$$

where $s_{i}: \xi_{i 2} \rightarrow \theta_{i}$ and $\sigma_{i}: \xi_{i 2} \rightarrow \kappa_{i}$ are $\mathcal{C}^{\infty}$ left inverses to the monomorphisms $\theta_{i} \rightarrow \xi_{i 2}$ and $\kappa_{i} \rightarrow \xi_{i 2}$. Of course

$$
\sum_{i=0}^{m}(-1)^{i}\left(\zeta_{i 1}, \zeta_{i 0}, f_{i}^{-1}\right)
$$

vanishes in $K_{0}^{\mathrm{rel}}(X)$ because of relations (iv) and (viii) (for the $j_{i}$ ). One obtains a complex $\left(\zeta_{\bullet}\right)$ of $\mathcal{C}^{\infty}$ vector bundles on $S^{1} \times X$ such that for any $t \in S^{1}$, the restriction $\left.\zeta_{\bullet}\right|_{\{t\} \times X}$ yields a resolution of the sheaf $\iota_{*} \mathcal{O}_{Y}\left(\left.\eta\right|_{\{t\} \times X}\right)$ on $X$. Now $\left(\zeta_{\bullet}\right)$ is easily seen to be a $\left(\mathcal{C}^{\infty}\right)$ resolution of $\iota_{*} \mathcal{O}_{S^{1} \times Y}(\eta)$ in the sense of [1], $\S 4(1)$, so that the topological $K$-theory class of $\left(\zeta_{\bullet}\right)$ is in fact the direct image in $K_{0}^{\mathrm{top}}\left(S^{1} \times X\right)$ of the topological $K$-theory class of $\eta$ in $K_{0}^{\mathrm{top}}\left(S^{1} \times Y\right)$ in the sense of [1], $\S 4$. (Note that the map $K_{0}^{\text {top }}\left(S^{1} \times Y\right) \longrightarrow K_{0}^{\text {top }}\left(S^{1} \times X\right)$ factors throw $K_{0}^{\text {top }}\left(S^{1} \times X, S^{1} \times(X \backslash Y)\right)$ where it is defined in [1].) From the assumptions made on $\eta_{0}, \eta_{1}$ and $f_{01}, \eta$ on $S^{1} \times Y$ defines a tivial class in $K_{1}^{\text {top }}(Y)$ so that $\zeta_{\bullet}$ on $S^{1} \times X$ also defines a trivial class in $K_{1}^{\text {top }}(X)$. Thus

$$
\sum_{i=0}^{m}(-1)^{i}\left[\left(\zeta_{i 0}, \zeta_{i 1}, g_{i 01}\right)+\left(\zeta_{i 1}, \zeta_{i 0}, f_{i}^{-1}\right)\right]
$$

vanishes in $K_{0}^{\mathrm{rel}}(X)$ and hence $\sum_{i=0}^{m}(-1)^{i}\left(\xi_{i 0}, \xi_{i 1}, g_{i 01}\right)$ also does as desired.

Note that the same argument shows the commutativity of the left square of the diagram.

If now the same element of $K_{0}^{\operatorname{def}}(Y)$ is represented in two different ways, that is two bundles $\eta^{\prime}$ and $\eta^{\prime \prime}$ on $\Sigma^{\prime} \times Y$ and $\Sigma^{\prime \prime} \times Y$ verify $\left.\left.\eta^{\prime}\right|_{\left\{q_{i}^{\prime}\right\} \times Y} \cong \eta^{\prime \prime}\right|_{\left\{q_{i}^{\prime \prime}\right\} \times Y}$ for $i=0$ and 1 , the isomorphisms $\left(k_{0}\right.$ and $\left.k_{1}\right)$ being holomorphic, and

$$
\left(\eta_{0}^{\prime}, \eta_{1}^{\prime}, f_{01}^{\prime}\right)=\left(\eta_{0}^{\prime \prime}, \eta_{1}^{\prime \prime}, f_{01}^{\prime \prime}\right) \quad \text { in } K_{0}^{\mathrm{rel}}(Y)
$$

with $f_{01}^{\prime \prime}=k_{1} \circ f_{01}^{\prime} \circ k_{0}^{-1}$. Consider two resolutions $\left(\xi_{\bullet}^{\prime}\right)$ on $\Sigma^{\prime} \times X$ and $\left(\xi_{\bullet}^{\prime \prime}\right)$ on $\Sigma^{\prime \prime} \times X$. From [10], démonstration du lemme 11, there is some "dominating" resolution $\left(\xi_{\bullet}^{(3)}\right)$ on $X$ of the sheaf $\iota_{*} \mathcal{O}_{Y}\left(\eta_{1}^{\prime}\right) \cong \iota_{*} \mathcal{O}_{Y}\left(\eta_{1}^{\prime \prime}\right)$ with graded epimorphisms $\xi_{\bullet}^{(3)} \stackrel{h_{\bullet}^{\prime}}{\rightarrow} \xi_{\bullet}^{\prime}$ and $\xi_{\bullet}^{(3)} \stackrel{h_{\bullet}^{\prime \prime}}{\rightarrow} \xi_{\bullet 1}^{\prime \prime}$. Call $\theta_{\bullet}^{\prime}$ and $\theta_{\bullet}^{\prime \prime}$ the kernels of these epimorphisms (they are of course exact sequences of vector bundles) and lift them trivially on $\Sigma^{\prime} \times X$ and $\Sigma^{\prime \prime} \times X$ respectively. Identify $\xi_{\bullet 1}^{\prime} \oplus \theta_{\bullet}^{\prime}$ with $\xi_{\bullet 1}^{\prime \prime} \oplus \theta_{\bullet}^{\prime \prime}$ throw $f_{\bullet}=\left(h_{\bullet}^{\prime} \oplus s_{\bullet}^{\prime}\right)^{-1} \circ\left(h_{\bullet}^{\prime \prime} \oplus s_{\bullet}^{\prime \prime}\right)$ where $s_{\bullet}^{\prime}$ and $s_{\bullet}^{\prime \prime}$ are $\mathcal{C}^{\infty}$ left inverses of the 
monomorphisms $\theta_{\bullet}^{\prime} \rightarrow \xi_{\bullet}^{(3)}$ and $\theta_{\bullet}^{\prime \prime} \rightarrow \xi_{\bullet}^{(3)}$. One gets a complex of vector bundles on $\gamma \times X$ where $\gamma$ is the path from $q_{0}^{\prime}$ to $q_{0}^{\prime \prime}$ obtained by gluing the path from $q_{0}^{\prime}$ to $q_{1}^{\prime}$ to the path from $q_{1}^{\prime \prime}$ to $q_{0}^{\prime \prime}$ by identifying $q_{1}^{\prime}$ and $q_{1}^{\prime \prime}$. Then the preceding construction can be performed with this complex of bundles on $\gamma \times X$ instead of the preceding $\left(\xi_{\bullet}\right)$ on some path from $q_{0}$ to $q_{1}$. This yields that:

$$
\begin{aligned}
\sum_{i=0}^{m}(-1)^{i}\left(\xi_{i 0}^{\prime}, \xi_{i 1}^{\prime}, g_{i 01}^{\prime}\right)+\sum_{i=0}^{m}(-1)^{i}\left(\xi_{i 1}^{\prime} \oplus \theta_{i}^{\prime}, \xi_{i 1}^{\prime \prime} \oplus \theta_{i}^{\prime \prime}, f_{i}\right) \\
+\sum_{i=0}^{m}(-1)^{i}\left(\xi_{i 1}^{\prime \prime}, \xi_{i 0}^{\prime \prime}, g_{i 01}^{\prime \prime}-1\right)
\end{aligned}
$$

vanishes in $K_{0}^{\text {rel }}(X)$. The central sum vanishes (from (iv) and (viii) as before) thus the direct images of $\left(\eta_{0}^{\prime}, \eta_{1}^{\prime}, f_{01}^{\prime}\right)=\left(\eta_{0}^{\prime \prime}, \eta_{1}^{\prime \prime}, f_{01}^{\prime \prime}\right)$ calculated from $\Sigma^{\prime}$ and $\xi_{\bullet}^{\prime}$ or $\Sigma^{\prime \prime}$ and $\xi_{\bullet}^{\prime \prime}$ coïncide. It is therefore independent on the choices of the couple $(\Sigma, \eta)$ and of the resolution.

The compatibility of the direct image map with relations (i) and (iii) defining $K_{0}^{\text {rel }}$ becomes obvious, the compatibility with relation (iv) is a consequence of the fact that a short exact sequence on $Y$ can be resolved by a complex of short exact sequences on $X$ (see [10], démonstration du lemme 12) and that all this can be realised on $\mathbb{P}^{1} \times Y$ and $\mathbb{P}^{1} \times X$ using the standard construction as in the proof of Proposition 6.4 above.

Finally, the commutativity of the right square is obvious.

6.7. General Riemann-Roch on $\boldsymbol{K}_{\mathbf{0}}^{\text {def }}$. - Before stating the RiemannRoch theorem, one has to take care of direct image on cohomology:

$$
\begin{aligned}
\iota_{*}: H^{\bullet}(Y) & \longrightarrow H^{\bullet}(X), \\
\alpha & \longmapsto \propto \wedge \delta_{Y},
\end{aligned}
$$

where $\delta_{Y}$ is the current of integration along $Y$. In fact the de Rham cohomology of a compact manifold can be calculated using currents instead of $\mathcal{C}^{\infty}$ differential forms and if the manifold is Kähler, the Fröhlicher spectral sequence on currents degenerates at $E_{1}$ as in the case of $\mathcal{C}^{\infty}$ differential forms. Thus the cohomology groups $H^{2 \bullet-1}\left(\wedge(X) / F_{\bullet}\right)$ can also be calculated using currents instead of $\mathcal{C}^{\infty}$ differential forms. Now $\delta_{Y}$ is an integral current of complex type $(\operatorname{codim} Y, \operatorname{codim} Y)$ on $X$ thus:

$$
\begin{aligned}
& \iota_{*}: H^{2 r-1}\left(\wedge(Y) / F_{r}\right) \longrightarrow H^{2 r+2 \operatorname{codim} Y-1}\left(\wedge(X) / F_{r+\operatorname{codim} Y}\right), \\
& \iota_{*}: H^{2 \bullet-1}\left(\wedge(Y) / F_{\bullet}\right) \longrightarrow H^{2 \bullet-1}\left(\wedge(X) / F_{\bullet}\right) .
\end{aligned}
$$

TheOREM. - For $\eta$ and $\xi$ as above

$$
\sum_{i=0}^{m}(-1)^{i} \mathcal{N}_{\mathrm{ch}}\left(\xi_{i 0}, \xi_{i 1}, g_{i 01}\right)=\iota_{*}\left(\mathrm{Td}^{-1}(N) \wedge \mathcal{N}_{\mathrm{ch}}\left(\eta_{0}, \eta_{1}, f_{01}\right)\right)
$$

TOME $130-2002-\mathrm{N}^{\mathrm{O}} 2$ 
where $\operatorname{Td}^{-1}(N) \in \bigoplus_{p} H^{p, p}(Y)$ is the inverse of the Todd class of the normal bundle $N$ of $Y$ in $X$.

COROLlaRy. - For any proper morphism $f: Y \rightarrow Z$ of projective manifolds one has for any $\Xi \in K_{0}^{\operatorname{def}}(Y)$

$$
\operatorname{Td}(T Z) \wedge \mathcal{N}_{\text {ch }}\left(f_{*} \Xi\right)=f_{*}\left(\operatorname{Td}(T Y) \wedge \mathcal{N}_{\text {ch }}(\Xi)\right)
$$

( $T Z$ and $T Y$ are the tangent bundles of $Z$ and $Y$ ).

Proof of corollary. - This is a direct consequence of Corollary 6.6, the above theorem and the classical technique of factorising any proper morphism into a closed immersion and a proper submersion.

Proof of the theorem. - Choose any Kähler metric on $X$ and $\Sigma$ and hermitian metrics $h^{\eta}$ on $\eta$ and $h^{\xi_{i}}$ on $\xi_{i}$ such that assumption $(A)$ of Bismut (see [5], Def. 1.5 or [8], Def. 1.1) is satisfied. Such metrics always exist according to [5], Prop. 1.6.

Bismut, Gillet and Soulé [8], Def. 2.4 constructed in the above situation two currents $T$ and $\gamma$ on $\Sigma \times X$ which verify the following equations of currents on $\Sigma \times X([8]$, Thm. 2.5):

$$
\begin{aligned}
& \frac{1}{2 \pi i}(\bar{\partial}-\partial) T=2 \gamma, \\
& \mathrm{d} \gamma=\sum_{i=0}^{m}(-1)^{i} \operatorname{ch}\left(\xi_{i}\right)-\mathrm{Td}^{-1}(N) \wedge \operatorname{ch}(\eta) \wedge \delta_{\Sigma \times Y},
\end{aligned}
$$

where Chern characters and Todd classes are explicit differential forms in Chern-Weil theory calculated with the hermitian compatible connexions associated to the chosen metrics, and $\delta_{\Sigma \times Y}$ is the current of integration along $\Sigma \times Y$ in $\Sigma \times X$. Moreover $T$ and $\gamma$ have their wave front set included in the dual of the real normal bundle of $\Sigma \times Y$ in $\Sigma \times X$ (which is nothing but the pull-back to $\Sigma \times X$ by the projection on $X$ of the normal bundle $N$ of $Y$ in $X$ ). This allows to integrate the latter formula along $\widetilde{q}$ to obtain the following equation of currents on $X$ :

$$
\begin{aligned}
& \left(\int_{\tilde{q}([0,1])} \sum_{i=0}^{m}(-1)^{i} \operatorname{ch}\left(\xi_{i}\right)\right)-\operatorname{Td}^{-1}(N) \wedge\left(\int_{\tilde{q}([0,1])} \operatorname{ch}(\eta)\right) \wedge \delta_{Y} \\
& \quad=\sum_{i=0}^{m}(-1)^{i} \overline{\operatorname{ch}}\left(\xi_{i 0}, \xi_{i 1}, g_{i 01}\right)-\mathrm{Td}^{-1}(N) \wedge \overline{\operatorname{ch}}\left(\eta_{0}, \eta_{1}, f_{01}\right) \wedge \delta_{Y} \\
& \quad=\mathrm{d}\left(\int_{\tilde{q}([0,1])} \gamma\right)+\left.\gamma\right|_{\left\{q_{1}\right\} \times X}-\left.\gamma\right|_{\left\{q_{0}\right\} \times X}
\end{aligned}
$$

but the constructions of $\gamma$ and $T$ are universal and functorial by pull-backs by transversal maps (see [8], Thm. 2.7). The maps $\left\{q_{0}\right.$ or $\left.q_{1}\right\} \times X \longrightarrow \Sigma \times X$ are obviously transversal in the sense of [8], Def. 2.6, so that $\left.T\right|_{\left\{q_{0}\right\} \times X}$ and 
$\left.T\right|_{\left\{q_{1}\right\} \times X}$ are (as $T$ is from [8], Thm. 2.5 and Def. 1.3) sums of currents of complex type $(p, p)$ and

$$
\begin{aligned}
\left.\gamma\right|_{\left\{q_{0}\right\} \times X} & =\left.\frac{1}{4 \pi i}(\bar{\partial}-\partial) T\right|_{\left\{q_{0}\right\} \times X} \\
& =\left.\frac{-1}{2 \pi i} \partial T\right|_{\left\{q_{0}\right\} \times X}+\left.\frac{1}{4 \pi i} \mathrm{~d} T\right|_{\left\{q_{0}\right\} \times X}
\end{aligned}
$$

is the sum of an exact current and of currents of complex types $(p+1, p)$. The same holds for $\left.\gamma\right|_{\left\{q_{1}\right\} \times X}$ and thus the classes of $\left.\gamma\right|_{\left\{q_{0}\right\} \times X}$ and $\left.\gamma\right|_{\left\{q_{1}\right\} \times X}$ vanish in $H^{2 \bullet-1}\left(\wedge(X) / F_{\bullet}\right)$. (44) then yields:

$$
\sum_{i=0}^{m}(-1)^{i} \mathcal{N}_{\mathrm{ch}}\left(\xi_{i 0}, \xi_{i 1}, g_{i 01}\right)=\mathrm{Td}^{-1}(N) \wedge \mathcal{N}_{\mathrm{ch}}\left(\eta_{0}, \eta_{1}, f_{01}\right) \wedge \delta_{Y}
$$

as desired.

REMARK 6.9. - For a closed immersion $\iota: Y \rightarrow X$ it is straightforward to define $\iota_{*}: K_{0}^{\mathrm{hom}}(Y) \rightarrow K_{0}^{\mathrm{hom}}(X)$, and this is also true for a proper submersion $\pi: X \rightarrow Y$. In the immersion case, the direct application of the RiemannRoch theorem without denominators for the total Chern class with values in Hodge-Deligne cohomology (or equivalently with values in the Chow groups and associated with theorem 1.1) will give some statement for classes with values in the intermediate jacobians. However, such a statement cannot exist for submersions since an element of $K_{0}^{\mathrm{rel}}(X)$ with vanishing $\mathcal{N}_{R \text { ch }}$ class in $\bigoplus_{r} J_{r}(X)$ need not lie in the image of $K_{1}^{\text {top }}(X)$ so that it does not vanish in $K_{0}^{\text {hom }}(X)$ and its image in $K_{0}^{\text {hom }}(Y)$ need not have vanishing $\mathcal{N}_{R \text { ch }}$ class in $\bigoplus_{r} J_{r}(Y)$.

Consider the subgroup $S R \operatorname{ch}\left(K_{1}^{\text {top }}(X)\right) \subset H^{\text {odd }}(X)$ and set

$$
\widehat{J}_{r}(X)=H^{2 r-1}\left(\wedge(X) / F_{r}\right) / \psi\left(S R \operatorname{ch}\left(K_{1}^{\mathrm{top}}(X)\right)\right)
$$

(and similarly for $Y$ ). One can remedy the preceding problem using $\bigoplus_{r} \widehat{J}_{r}$ instead of $\bigoplus_{r} J_{r}: \mathcal{N}_{R \text { ch }}$ is obviously defined on $K_{0}^{\text {hom }}$ with values in $\bigoplus_{r} \widehat{J}_{r}$ and one obtains for any $\Xi \in K_{0}^{\mathrm{hom}}(X)$

$$
\mathcal{N}_{R \operatorname{ch}}\left(\pi_{*} \Xi\right)=\widehat{\pi}_{*}\left(\mathcal{N}_{R \operatorname{ch}}(\Xi)\right)
$$

for $\widehat{\pi}_{*}: \bigoplus_{r} \widehat{J}_{r}(X) \rightarrow \bigoplus_{s} \widehat{J}_{s}(Y)$ obtained as follows: call $N_{Y}$ the operator on $H^{\text {odd }}(Y)$ which multiplies $(2 s-1)$-degree forms by $(-1)^{s-1}(s-1)$ !, then

$$
\widehat{\pi}_{*} \alpha_{r}=N_{Y}\left(\int_{X / Y} \operatorname{Td}(T X / Y) \wedge \frac{(-1)^{r-1} \alpha_{r}}{(r-1) !}\right)
$$

for $\alpha_{r} \in \widehat{J}_{r}(X)$ (of course the fact that $\pi_{*} \operatorname{maps} S_{R \mathrm{ch}}\left(K_{1}^{\mathrm{top}}(X)\right.$ to $S_{R \mathrm{ch}}\left(K_{1}^{\mathrm{top}}(Y)\right.$ is ensured by the Riemann-Roch theorem for $\left.K_{1}^{\text {top }}\right)$. Of course there is now no more corresponding statement for closed immersions (which would then provide a Riemann-Roch theorem on $K_{0}^{\text {hom }}$ for any proper morphism); in fact such a

TOME $130-2002-\mathrm{N}^{\mathrm{O}} 2$ 
statement would be contained in the definition of direct image morphisms for Karoubi's multiplicative $K$-theory (which are not yet available).

\section{BIBLIOGRAPHY}

[1] Atiyah (M.F.) \& Hirzebruch (F.) - Analytic cycles on complex manifolds, Topology, t. 1 (1962), pp. 25-45.

[2] Atiyah (M.F.) \& Singer (I.M.) - The index of elliptic operators. IV, Ann. of Math., t. 93 (1971), pp. 119-138.

[3] Berline (N.), Getzler (E.) \& Vergne (M.) - Heat kernels and Dirac operators, Grundlehre der Mathematischen Wissenschaften, vol. 298, Springer-Verlag, Berlin, 1992.

[4] Bismut (J.-M.) - The Atiyah-Singer index theorem for families of Dirac operators: two heat equation proofs, Invent. Math., t. 83 (1986), pp. 91151.

[5] _ Superconnexion currents and complex immersions, Invent. Math., t. 99 (1990), pp. 59-113.

[6] Quillen metrics and singular fibres in arbitrary relative dimension, J. Algebraic Geom., t. 6 (1997), pp. 19-149.

[7] Bismut (J.-M.), Gillet (H.) \& Soulé (C.) - Analytic Torsion and Holomorphic Determinant Bundles II. Direct images and Bott-Chern forms, Comm. Math. Phys., t. 115 (1988), pp. 79-126.

[8] _ Bott-Chern currents and complex immersions, Duke Math. J., t. 60 (1990), pp. 255-284.

[9] Bismut (J.-M.) \& KöHLeR (K.) - Higher analytic torsion forms for direct images and anomaly formulas, J. Algebraic Geom., t. 1 (1992), pp. 647-684.

[10] Borel (A.) \& Serre (J.-P.) - Le théorème de Riemann-Roch, Bull. Soc. Math. France, t. 86 (1958), pp. 97-136.

[11] Brylinski (J.-L.) - Comparison of the Beilinson-Chern classes with the Chern-Cheeger-Simons classes, in Advances in Geometry, Progr. Math., vol. 172, Birkhäuser Boston, Boston, MA, 1999, pp. 95-105.

[12] Cheeger (J.) \& Simons (J.) - Differential characters and geometric invariants, in Geometry and Topology (College Park, Md., 1983/84), Lecture Notes in Math., vol. 1167, Springer, Berlin, 1985, pp. 50-80.

[13] Chern (S.S.) \& Simons (J.) - Characteristic forms and geometric invariants, Ann. of Math., t. 99 (1974), pp. 48-69.

[14] Deligne (P.) - Théorie de Hodge II, Inst. Hautes Études Sci. Publ. Math., t. 40 (1971), pp. 5-57.

[15] El Zein (F.) \& ZuCKer (S.) - Extendability of normal functions associated to algebraic cycles, in Topics in transcendental algebraic geometry 
(Princeton, N.J., 1981/82), Ann. Math. Studies, vol. 106, Princeton Univ. Press, Princeton, NJ, 1984, pp. 269-288.

[16] Esnault (H.) \& SRinivas (V.) - Chern classes of vector bundles with holomorphic connections on a complete smooth complex variety, J. Diff. Geom., t. 36 (1992), pp. 257-267.

[17] Esnault (H.) \& Viehweg (E.) - Deligne-Beilinson cohomology, in Beilinson's conjectures on special values of L-functions, Perspect. Math., vol. 4, Academic Press, Boston, MA, 1988, pp. 43-91.

[18] Gillet (H.) - Riemann-Roch theorems for higher algebraic K-theory, Adv. in Math., t. 40 (1981), pp. 203-289.

[19] Leligne homology and Abel-Jacobi maps, Bull. Amer. Math. Soc., t. 10 (1984), pp. 285-288.

[20] Gillet (H.) \& Grayson (D.) - The loop space of the q-construction, Illinois J. Math., t. 31 (1987), pp. 574-597.

[21] Gillet (H.) \& Soulé (C.) - Arithmetic Chow groups and differential characters, in Algebraic K-theory: conections with geometry and topology (Jardine (J.F.) \& Snaith (V.P.), eds.), Kluwer Academic, Dordrecht, 1989, pp. 30-68.

[22] Greiner (P.) - An asymptotic expansion for the heat equation, Arch. Rational Mech. Anal., t. 41 (1971), pp. 163-218.

[23] Grothendieck (A.) - La théorie des classes de Chern, Bull. Soc. Math. France, t. 86 (1958), pp. 137-154.

[24] Iliev (A.) \& MarkusheViCh (D.) - The Abel-Jacobi map for a cubic threefold and periods of Fano threefolds of degree 14, Doc. Math., t. 5 (2000), pp. 23-47.

[25] Jannsen (U.) - Deligne homology, Hodge-D-conjecture and motives, in Beilinson's conjectures on special values of L-functions, Perspect. Math., vol. 4, Academic Press, Boston, MA, 1988, pp. 305-372.

[26] Jouanolou (J.-P.) - Une suite exacte de Mayer-Vietoris en K-théorie algébrique, in Algebraic K-theory, I: Higher $K$-theories, Lecture Notes in Math., vol. 341, Springer-Verlag, New York, 1973, pp. 293-376.

[27] Karoubi (M.) - K-theory, an Introduction, Grundlehre der Mathematischen Wissenschaften, vol. 226, Springer-Verlag, Berlin, 1978.

[28] _ Homologie cyclique et K-théorie, Astérisque, vol. 149, Société Mathématique de France, 1987.

[29] - Théorie générale des classes caractéristiques secondaires, KTheory, t. 4 (1990), pp. 55-87.

[30] Classes caractéristiques de fibrés feuilletés, holomorphes ou algébriques, K-Theory, t. 8 (1994), pp. 153-211.

[31] Lewis (J.D.) - A survey of the Hodge conjecture, Introductory lectures in transcendental algebraic geometry, Université de Montréal, Centre de Recherches Mathématiques, Montréal, QC, 1991. 
[32] Markushevich (D.) \& Tikhomirov (A.S.) - The Abel-Jacobi map of a moduli component of vector bundles on the cubic threefold, J. Alg. Geom., t. 10 (2001), pp. 37-62.

[33] Mumford (D.) - Abelian varieties, Tata institute of Fundamental Research Studies in Mathematics, vol. 5, Published for the Tata Institute of fundamental Research, Bombay; Oxford University Press, London, 1970.

[34] Murre (J.) - Un résultat en théorie des cycles algébriques de codimension 2, C. R. Acad. Sci. Paris, Sér. I Math., t. 296 (1983), pp. 981-984.

[35] NAdel (A.) - Invariants for holomorphic vector bundles, Math. Ann., t. 309 (1997), pp. 37-52.

[36] Roessler (D.) - Analytic torsion for cubes of vector bundles and Gillet's Riemann-Roch theorem, J. Alg. Geom., t. 8 (1999), pp. 497-518.

[37] Yoshioka (K.) - Albanese map of moduli of stable sheaves on abelian surfaces, Preprint, math.AG/9901013, 1999.

[38] Some notes on the moduli of stable sheaves on elliptic surfaces, Nagoya Math. J., t. 154 (1999), pp. 73-102.

[39] ZuCKer (S.) - The Cheeger-Simons invariant as a Chern class, in Algebraic analysis, geometry, and number theory (Baltimore, MD, 1988), Johns Hopkins Univ. Press, Baltimore, MD, 1989, pp. 397-417. 